\title{
Studies of the performance of the ATLAS detector using cosmic-ray muons
}

\author{
The ATLAS Collaboration ${ }^{\star}$ \\ CERN, 1211 Geneva 23, Switzerland
}

Received: 1 December 2010 / Revised: 27 January 2011 / Published online: 29 March 2011

(C) CERN for the benefit of the ATLAS collaboration 2011. This article is published with open access at Springerlink.com

\begin{abstract}
Muons from cosmic-ray interactions in the atmosphere provide a high-statistics source of particles that can be used to study the performance and calibration of the ATLAS detector. Cosmic-ray muons can penetrate to the cavern and deposit energy in all detector subsystems. Such events have played an important role in the commissioning of the detector since the start of the installation phase in 2005 and were particularly important for understanding the detector performance in the time prior to the arrival of the first LHC beams. Global cosmic-ray runs were undertaken in both 2008 and 2009 and these data have been used through to the early phases of collision data-taking as a tool for calibration, alignment and detector monitoring. These large datasets have also been used for detector performance studies, including investigations that rely on the combined performance of different subsystems. This paper presents the results of performance studies related to combined tracking, lepton identification and the reconstruction of jets and missing transverse energy. Results are compared to expectations based on a cosmic-ray event generator and a full simulation of the detector response.
\end{abstract}

\section{Introduction}

The ATLAS detector [1] was constructed to provide excellent physics performance in the difficult environment of the Large Hadron Collider (LHC) at CERN [2], which will collide protons at center-of-mass energies up to $14 \mathrm{TeV}$, with unprecedented luminosity. It is designed to be sensitive to any experimental signature that might be associated with physics at this new high-energy frontier. This includes precision measurements of high $p_{\mathrm{T}}$ leptons and jets, as well as large transverse-energy imbalances attributable to the production of massive weakly interacting particles. Such particles are predicted in numerous theories of physics beyond

^e-mail: atlas.secretariat@cern.ch the Standard Model, for example those invoking weak-scale supersymmetry or the existence of large extra dimensions.

Prior to the start of data-taking, understanding of the expected performance of individual subsystems relied on beam test results and on detailed GEANT4 [3, 4] simulations [5], including the modeling of inactive material both in the detector components and in the detector services and support structure. While extensive beam testing provided a great deal of information about the performance of the individual detector subsystems, a detailed understanding of the full detector could only be achieved after the system was in place and physics signals could be used for performance studies and for validation or tuning of the simulation.

In both 2008 and 2009 the ATLAS detector collected large samples of cosmic-ray events. These extended periods of operation allowed for the training of shift crews, the exercising of the trigger and data acquisition systems as well as of other infrastructure such as the data-handling system, reconstruction software, and tools for hardware and data-quality monitoring. The large data samples accumulated have also been used for a number of commissioning studies. Because cosmic-ray muons interact with the detector mainly as minimum-ionizing particles (MIPs), most traverse all of the subdetectors along their flight path. So, in addition to subdetector-specific cosmic-ray studies, these cosmic-ray data samples provide the first opportunity to study the combined performance of different detector components. Subsystem-specific cosmic-ray commissioning results have been documented in a series of separate publications [6-9]. This paper presents the results of studies relevant to combined tracking performance, lepton identification and calorimeter performance for the reconstruction of jets and missing transverse energy. Where simulation results are available, results are compared to expectations based on a dedicated cosmic-ray event generator, implemented in the detector simulation. 


\section{The ATLAS detector}

The ATLAS detector is described in detail elsewhere [1] and illustrated in Fig. 1. ATLAS uses a right-handed coordinate system with its origin at the nominal interaction point (IP). The beam direction defines the $z$-axis, the positive $x$-axis points from the IP towards the center of the LHC ring and the positive $y$-axis points upwards. Cylindrical coordinates $(r, \phi)$ are used in the transverse plane and the pseudorapidity $\eta$ is defined in terms of the polar angle $\theta$ as $\eta=-\ln \tan (\theta / 2)$.

The ATLAS detector is made up of a barrel region and two endcaps, with each region consisting of several detector subsystems. Closest to the interaction point is the Inner Detector (ID), which performs charged particle tracking out to $|\eta|$ of 2.5. It consists of two silicon detectorsthe Pixel Detector and the SemiConductor Tracker (SCT) and the Transition Radiation Tracker (TRT), all immersed in a $2 \mathrm{~T}$ axial magnetic field provided by a superconducting solenoid magnet. The TRT is based on individual drift tubes with radiators, which provide for electron identification. The ID is surrounded by barrel and endcap liquid argon electromagnetic (EM) calorimeters which provide coverage out to $|\eta|$ of 3.1. These, in turn, are surrounded by hadronic calorimeters. In the barrel region, the Tile Calorimeter is composed of steel and scintillating tiles, with a central barrel and two extended-barrel regions providing coverage out to $|\eta|$ of 1.7. In the endcap region the Hadronic Endcap Calorimeter (HEC) is based on liquid argon and covers the region $1.5<|\eta|<3.2$. The calorimetric coverage is extended into the region $3.2<|\eta|<4.9$ by a liquid argon Forward Calorimeter (FCal) which occupies the same cryostat as the endcap EM calorimeter and the HEC. Beyond the calorimeter system is the Muon Spectrometer (MS), which relies on a set of massive superconducting air-core toroid magnets to produce a toroidal magnetic field in the barrel and endcap regions. In both regions, planes of interleaved muon detectors provide tracking coverage out to $|\eta|$ of 2.7 and triggering to $|\eta|$ of 2.4. The tracking studies presented in this paper are restricted to the barrel region of the detector, where precision measurements of the $(r, z)$ hit coordinates are provided by the Monitored Drift Tube (MDT) system. The remaining $\phi$ coordinate is measured by the Resistive Plate Chambers, or RPCs, which are primarily used for triggering.

ATLAS employs a three-level trigger system, with the Level-1 (L1) trigger relying primarily on information from the Muon and Calorimeter systems. For cosmic-ray running there was additionally a TRT-based trigger at L1 [10]. There is also a trigger based on signals from scintillators mounted in the endcap region, which are intended for triggering of collision events during the initial low-luminosity data-taking. This, however, plays no significant role in the triggering of cosmic-ray events. For the MS, the triggering in the barrel region of the detector is based on hits in the RPCs; in the endcap region, the Thin Gap Chambers (TGCs) are used. The L1 Calorimeter trigger (L1Calo) is based on analog sums provided directly from the calorime-

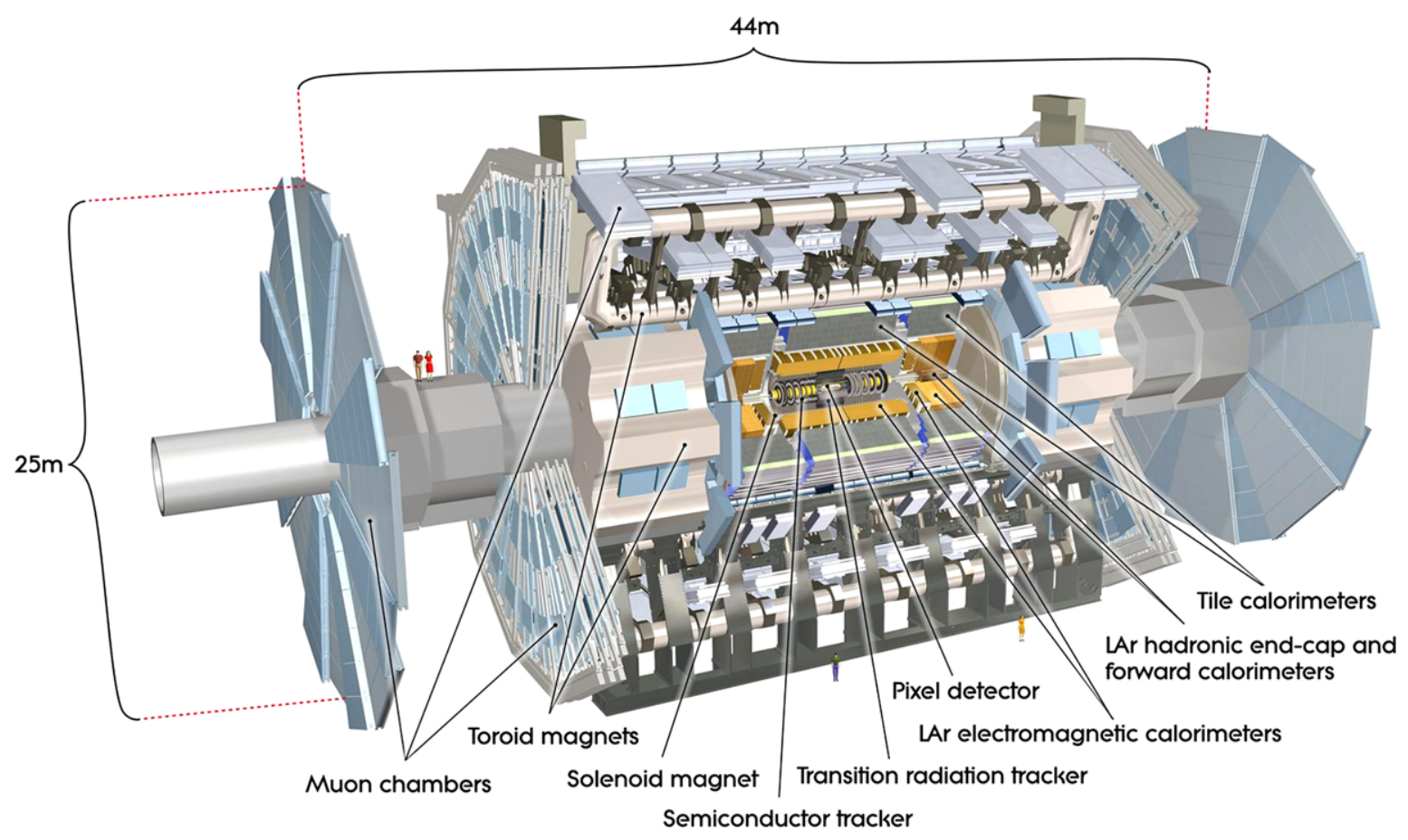

Fig. 1 The ATLAS detector and subsystems 
ter front-end readout, from collections of calorimeter cells forming roughly projective trigger towers. In each case, the L1 trigger identifies a region of interest (ROI) and information from this ROI is transmitted to L2. In normal operation, events accepted by the L2 trigger are sent to the Event Filter which performs the L3 triggering, based on full event reconstruction with algorithms similar to those used offline. The L2 and L3 trigger systems are jointly referred to as the High Level Trigger, or HLT. For the cosmic-ray data taking, events were triggered only at L1. Information from the HLT was used only to split the data into different samples.

\subsection{Tracking in ATLAS}

The two tracking systems, the ID and the MS, provide precision measurements of charged particle tracks. Reconstructed tracks are characterized by a set of parameters $\left(d_{0}, z_{0}, \phi_{0}\right.$, $\left.\theta_{0}, q / p\right)$ defined at the perigee, the point of closest approach of the track to the $z$-axis. The parameters $d_{0}$ and $z_{0}$ are the transverse and longitudinal coordinates of the perigee, $\phi_{0}$ and $\theta_{0}$ are the azimuthal and polar angles of the track at this point, and $q / p$ is the inverse momentum signed by the track charge. Analyses typically employ track quality cuts on the number of hits in a given tracking subsystem. The track reconstruction algorithms account for the possibility of energy loss and multiple scattering both in the material of the tracking detector itself, and in the material located between the tracking system and the particle production point. For the combined tracking of muons, which reconstructs the particle trajectories through both the ID and the MS, this requires an accurate modeling of the energy losses in the calorimeter. This will be discussed in Sect. 4.2.

\section{Cosmic-ray events in ATLAS}

Cosmic rays in ATLAS come mostly from above, and arrive mainly via two large access shafts used for the detector installation, as illustrated in Fig. 2.

In proton-proton collisions, the actual beam-spot position varies from the nominal IP by distances that are of order $\mathrm{mm}$ in the transverse plane and $\mathrm{cm}$ along the beam direction. Tracks produced in proton-proton collisions at the IP are said to be projective, that is, emanating from (or near, in the case of particles arising from secondary vertices) the IP. Cosmic-ray muons passing through the volume of the detector do not normally mimic such a trajectory. However, in a large sample of events, some do pass close to the center of the detector. By placing requirements on track impact parameters with respect to the nominal IP, it is possible to select a sample of approximately projective muons from those passing through the barrel region of the detector. Such cosmic-ray muons are referred to below

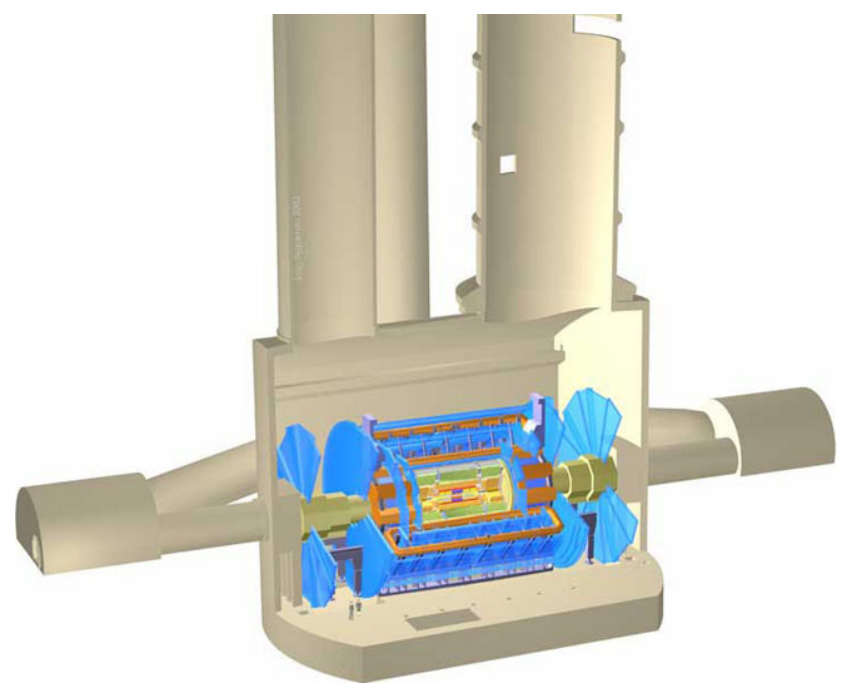

Fig. 2 The ATLAS detector in the experimental cavern. Above the cavern are the two access shafts used for the detector installation

as pseudo-projective. Due to the typical downward trajectory of the incoming cosmic-ray muons this cannot be done for those passing through the endcap region. For that reason, for those analyses presented here that rely on tracking, there is a requirement that the muons pass through the Inner Detector, which occupies a volume extending to about $1.15 \mathrm{~m}$ in radius and $\pm 2.7 \mathrm{~m}$ in $z$. The rate of such cosmicray muons is of order several Hz. Most analyses further restrict the acceptance to the barrel region of the ID, which has a smaller extent, in $z$, of $\pm 71.2 \mathrm{~cm}$. Some analyses additionally place requirements on the presence of hits in the SCT or Pixel detectors, further restricting the volume around the nominal IP through which the cosmic-ray muons are required to pass. Track-based event selection criteria are not applied in the case of the jet and missing transverse energy studies presented in Sect. 5, which focus on the identification of fake missing transverse energy due to cosmic-ray events or to cosmic-ray interactions that overlap with triggered events. While calorimeter cells are approximately projective towards the IP, ${ }^{1}$ energy deposits in the calorimeter can come from muons that pass through the calorimeter at any angle, including, for example, the highly non-projective up-down trajectory typical of cosmic muons passing through the endcap. While muons usually traverse the detector as MIPs, leaving only small energy deposits along their paths, in rare events they leave a larger fraction of their energy in the detector, particularly in the case of energy losses via bremsstrahlung. These can be particularly important in the case of high-energy muons, which can lose a significant amount of energy between the two tracking detectors. Such

\footnotetext{
${ }^{1}$ This is not the case for the FCal, which covers $3.2<|\eta|<4.9$, but that is not relevant to the analyses presented here.
} 
events have been previously exploited for pulse shape studies of the LAr calorimeter and as a source of photons used to validate the photon-identification capabilities of the ATLAS EM calorimeter [7, 11].

The reconstruction of cosmic-ray events is also complicated by the fact that they occur at random times with respect to the $40 \mathrm{MHz}$ readout clock, which is synchronized to the LHC clock during normal operation. For each subsystem, reconstruction of these events therefore first requires some measure of the event time with respect to the readout clock. An added complication, particularly for tracking, is that in the upper half of the detector, cosmic-ray muons travel from the outside in, rather than from the inside out, as would be the case for collisions. These differences can be addressed in the event reconstruction and data analysis. The modifications required for reconstruction of these events in the different detector components are discussed in the subsystemspecific cosmic-ray commissioning papers [6-9].

\subsection{Data samples}

ATLAS recorded data from global cosmic-ray runs during two extended periods, one in the fall of 2008 and another in the summer and fall of 2009. The analyses presented in this paper are each based on particular subsets of the available data.

For studies involving only the calorimeter, events triggered by L1Calo are used. Studies relying on tracking require that both the MS and ID were operational, and that the associated toroidal and solenoid fields both were at nominal strength. All L1-triggered events taken under those conditions were checked for the presence of a track in the ID. Events with at least one such track were streamed by the HLT to what is referred to here as the Pseudo-projective Cosmic-ray Muon (PCM) dataset, which forms the basic event sample for all of the studies presented in Sect. 4. These events are mainly triggered at L1 by the RPCs. Hundreds of millions of cosmic-ray events were recorded during the 2008 and 2009 cosmic-ray runs. However, the requirement of a track in the Inner Detector reduces the available statistics dramatically, as does the requirement of nominal magnetic field strengths for the MS and ID, which is necessary for studies of the nominal tracking performance.

\subsection{Cosmic-ray event simulation}

Cosmic-ray events in ATLAS are simulated using a dedicated event generator and the standard GEANT4 detector simulation, with the modeling of the readout electronics adapted to account for the difference in timing. The simulation includes the cavern overburden, the layout of the access shafts and an approximation of the material of the surface buildings. The event generator is based on flux calculations in reference [12] and uses a standard cosmic-muon momentum spectrum [13]. Single muons are generated near ground level, above the cavern in a $600 \mathrm{~m} \times 600 \mathrm{~m}$ region centered above the detector, with angles up to $70^{\circ}$ from vertical. Muons pointing to the cavern volume are propagated through up to $100 \mathrm{~m}$ of rock overburden, using GEANT4. Measurements of the cosmic-ray flux at different positions in the cavern were used to validate the predictions of this simulation [14]. Once a muon has been propagated to the cavern, additional filters are applied; only events with at least one hit in a given volume of the detector are retained, depending on the desired event sample. Note that only single-muon cosmic-ray events are simulated. No attempt is made to model events in which cosmic-ray interactions produce an air shower that can deposit large amounts of energy in the detector. However, the rate of such events (in data) has been shown to be sufficiently low that they do not produce significant discrepancies in, for example, the agreement between data and Monte Carlo (MC) for the distribution of the summed transverse energy in cosmic-ray events [15].

\section{Lepton identification and reconstruction studies using cosmic-ray events}

Cosmic-ray muons are an important tool for the commissioning of the muon spectrometer, which is the largest ATLAS subsystem, occupying over $95 \%$ of the total detector volume. As the rate of production of high- $p_{\mathrm{T}}$ muons in collision events is rather low, the cosmic-ray data will continue to be relevant to the MS commissioning for some time to come. ATLAS continues to record data from cosmic-ray interactions when LHC beams are not present.

While the cosmic rays are primarily a source of muons, analysis of these data also allows for checks of the algorithms used to identify other leptons. The cosmic-ray muons serve as a source of electrons, mainly $\delta$-electrons but with smaller contributions from the conversion of muon bremsstrahlung photons and muon decays in flight. The identification of a sample of electrons allowed for an examination of the performance of the electron identification algorithms, prior to first collisions. Similarly, although no $\tau$-leptons are expected in the cosmic-ray data sample, the tools designed for $\tau$-identification have been exercised using these data and checked against the simulation.

The analyses discussed in this section rely on the PCM dataset described earlier, which contains cosmic-ray muon events with tracks reconstructed in the ID. Most analyses also require the presence of hits in the Pixel Detector. These differ slightly for different analyses, as will be described below. 


\subsection{Combined muon tracking performance}

This section describes studies of the performance of the combined tracking for muons, using cosmic-ray data recorded in 2009. The investigation uses the PCM dataset in order to have tracks that resemble, as much as possible, tracks from collision data. Selected events are required to have a topology consistent with that expected for the passage of a cosmic-ray muon through the detector, which is illustrated by a typical event in Fig. 3. The requirements are:

- exactly 1 track reconstructed in the ID

- 1 or 2 tracks reconstructed in the MS

- exactly 1 combined track crossing both subdetectors

A special ID pattern recognition algorithm was used to reconstruct cosmic-ray muons as single tracks. Because of the topology of these events, the analysis is restricted to the barrel region of the detector. Good quality ID and MS tracks are ensured using requirements on the number of hits in the different subsystems. Events are required to have been triggered by the RPC chambers, since these also provide measurements along the $\phi$ coordinate ( $\phi$ hits), which is not measured by the MDTs. Following the procedure used in the ID commissioning with cosmic-ray muons [6], a requirement is also placed on the timing from the TRT, to ensure that the event was triggered in a good ID time window.

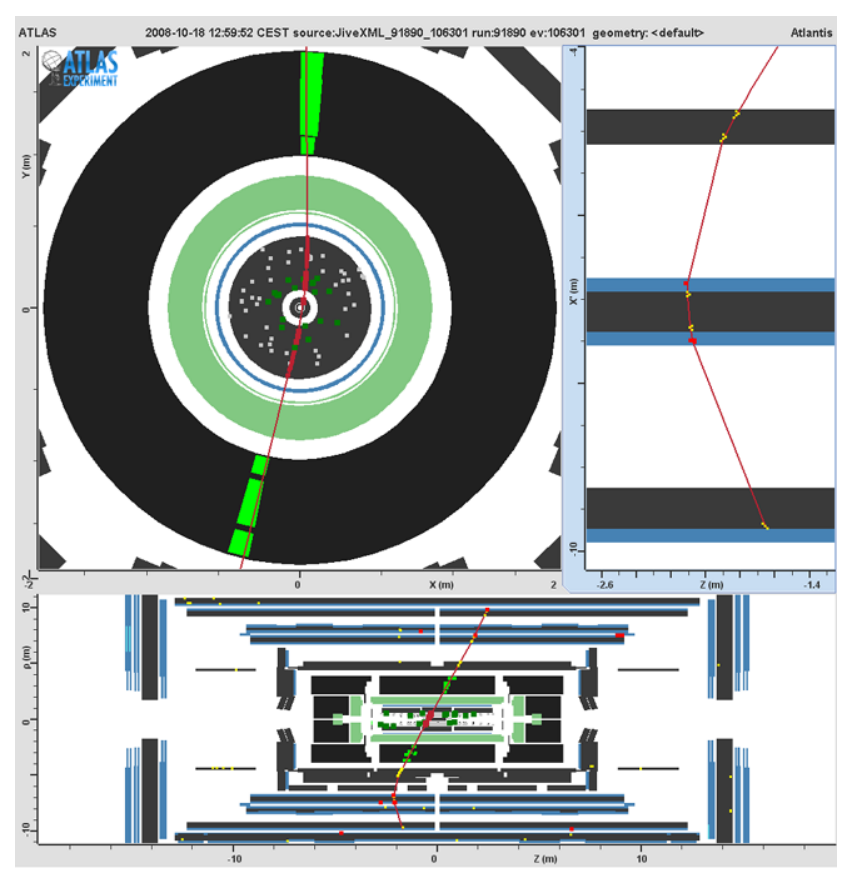

Fig. 3 Event display of a cosmic-ray muon crossing the entire ATLAS detector, close to the nominal IP, leaving hits in all tracking subsystems and significant energy deposits in the calorimeter. The upper left view shows the projection into the $r \phi$ plane. The lower plot shows the projection in the $r z$ plane. The upper right projection is a longitudinal slice through the central part of the Muon Spectrometer at the $\phi$ value of the MDT planes in which the muon hits were recorded
The track parameter resolutions for Combined Muon (CM) tracks have been investigated in the same manner as used for similar studies of the ID [6] and MS [9] performance, by comparing the two reconstructed tracks left by a single cosmic-ray muon passing through the upper and then the lower half of the detector. In the case of the ID and combined tracks, this involves separately fitting the hits in these two regions, to form what are referred to below as "split tracks" from the track created by the passage of a single muon.

Prior to a study of combined tracking, it is necessary to establish that the relative alignment of the two tracking systems is adequate. Checks were performed by comparing the track parameters for standalone tracks reconstructed by the two separate tracking systems, in the upper and lower halves of ATLAS. Tracks in the MS were reconstructed using a least-squares method that directly incorporates the effects of the material that sits between the MS detector planes and the point at which the track parameters are defined [16]. ID tracking was also performed by standard tracking algorithms $[17,18]$.

The alignment check relies on the study of three different classes of tracks: split ID tracks, MS standalone tracks, and split CM tracks. In what follows these will be referred to simply as ID, MS and CM tracks, respectively. Different quality cuts are placed on the three track types. For ID and CM tracks $\left|d_{0}\right|$ and $\left|z_{0}\right|$ are required to be less than $400 \mathrm{~mm}$ and $500 \mathrm{~mm}$ respectively. For MS tracks, for which these parameters must be extrapolated from the MS back to the perigee, the requirements are $\left|d_{0}\right|<1000 \mathrm{~mm}$ and $\left|z_{0}\right|<2000 \mathrm{~mm}$. ID and CM tracks are required to have at least 1, 6 and 20 hits in the Pixel, SCT and TRT detectors, respectively. MS and CM tracks are required to have hits in all three MS layers, with more than four RPC hits, at least two of which are $\phi$ hits, and a $\chi^{2}$ per degree of freedom less than 3 . All tracks are required to have momentum larger than $5 \mathrm{GeV}$.

Figure 4 shows the correlation between the $\phi_{0}$ and $\theta_{0}$ parameters determined from MS and ID tracks in the bottom half of ATLAS. Very good consistency is evident and similar results are obtained in the other hemisphere. The level of agreement between the two systems is better quantified by distributions of the difference between the track parameters obtained from the two systems. These are shown in Fig. 5 for $d_{0}, z_{0}, \phi_{0}$ and $\theta_{0}$, separately for tracks in the upper and lower halves of the detector. The somewhat narrower distributions obtained from the upper half of the detector are attributed to the higher average momentum of the cosmic-ray muons in this part of the detector, since those in the bottom have lost energy passing through the lower half of the calorimeter before reaching the MS. Small biases are observed for the $d_{0}$ and $\phi_{0}$ parameters. These are consistent with a slight translational misalignment between the MS and ID that is of order 
Fig. 4 Correlations between the track parameters $\phi_{0}$ and $\theta_{0}$ obtained from standalone ID and MS tracks, in the bottom half of ATLAS
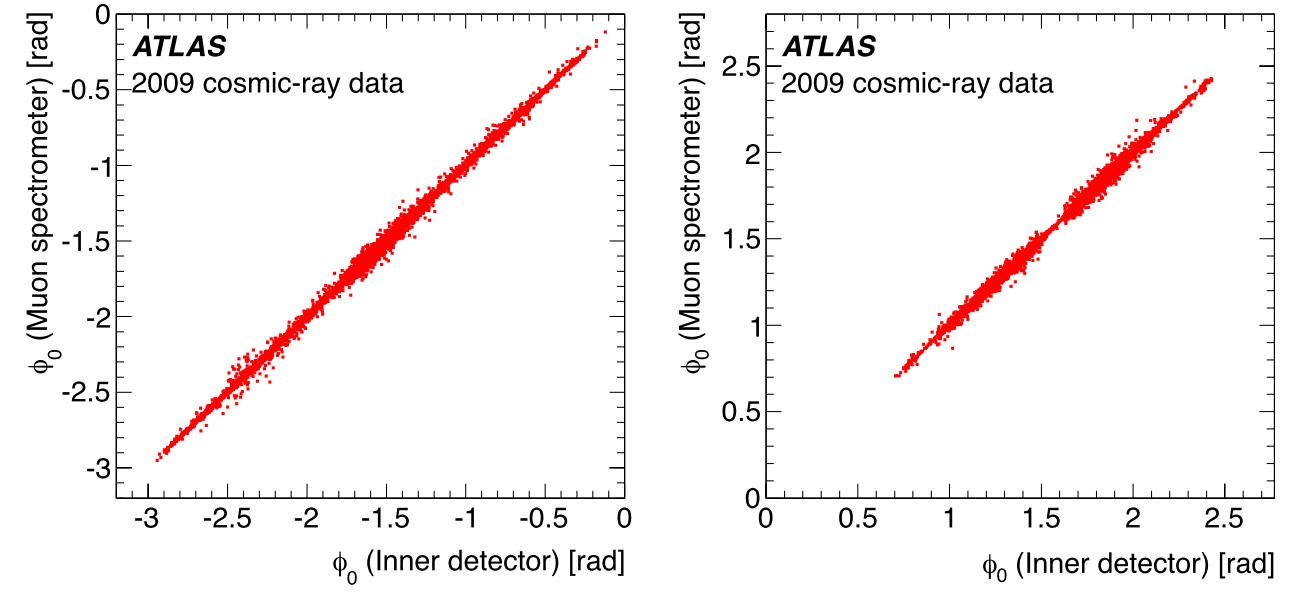
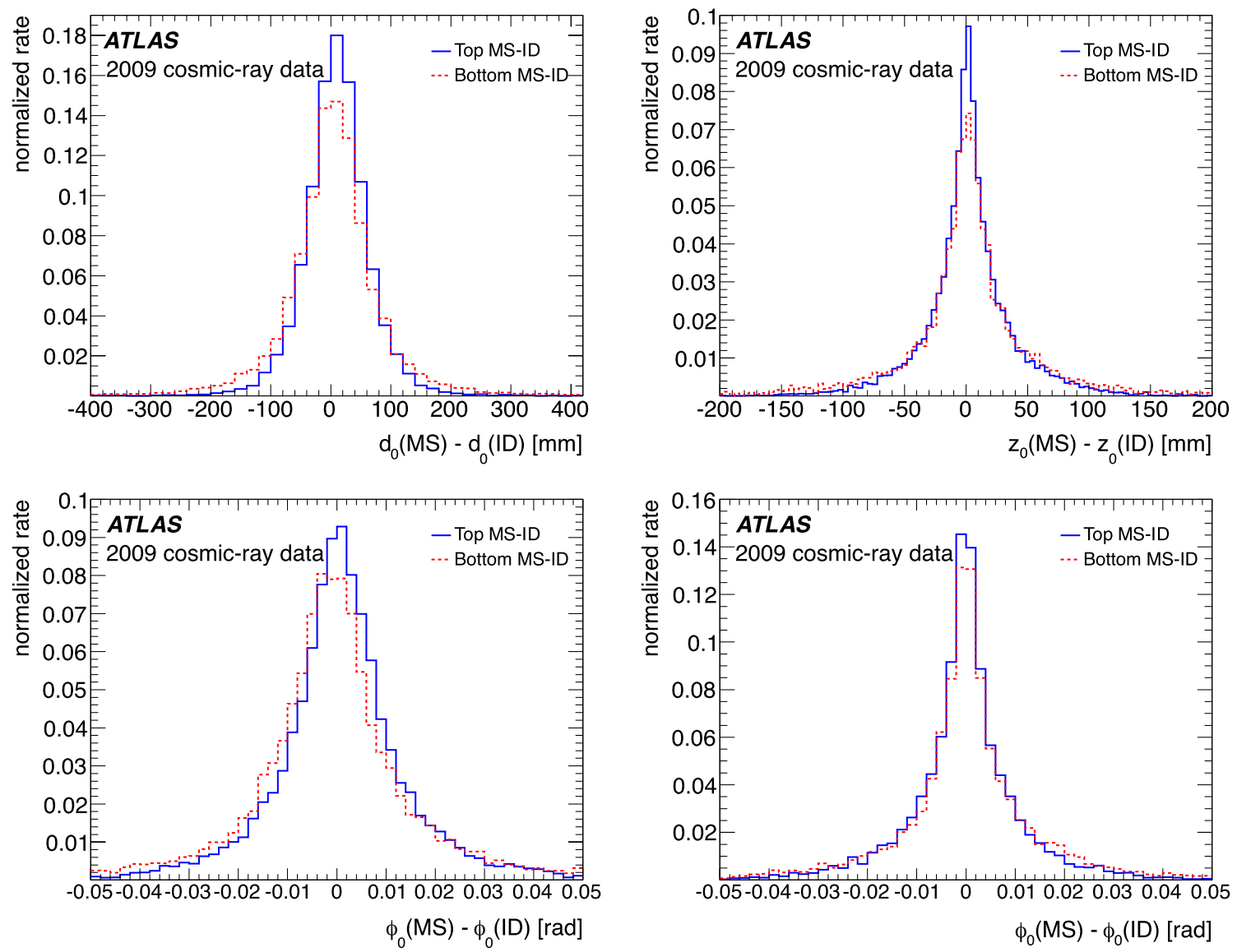

Fig. 5 Difference distributions of the track parameters, $d_{0}, z_{0}, \phi_{0}$ and $\theta_{0}$ obtained from standalone ID and MS tracks, for the top and bottom halves of the detector

$1 \mathrm{~mm}$. However, the combined tracking study presented below was performed without any relative ID-MS alignment corrections.

The track parameter resolutions for combined tracking have been investigated in the manner discussed above, using $\mathrm{CM}$ tracks passing through the barrel part of the detector, which are split into separate tracks in the upper and lower halves. The two resulting tracks are then fitted using the same combined track fit procedure. For studies of the angular and impact parameter resolution, the track quality cuts are tightened somewhat, with the requirements of at least two pixel hits, $\left|d_{0}\right|<100 \mathrm{~mm}$ and $\left|z_{0}\right|<400 \mathrm{~mm}$. An estimate of the resolution on each track parameter, $\lambda$, is obtained from the corresponding distribution of the difference 

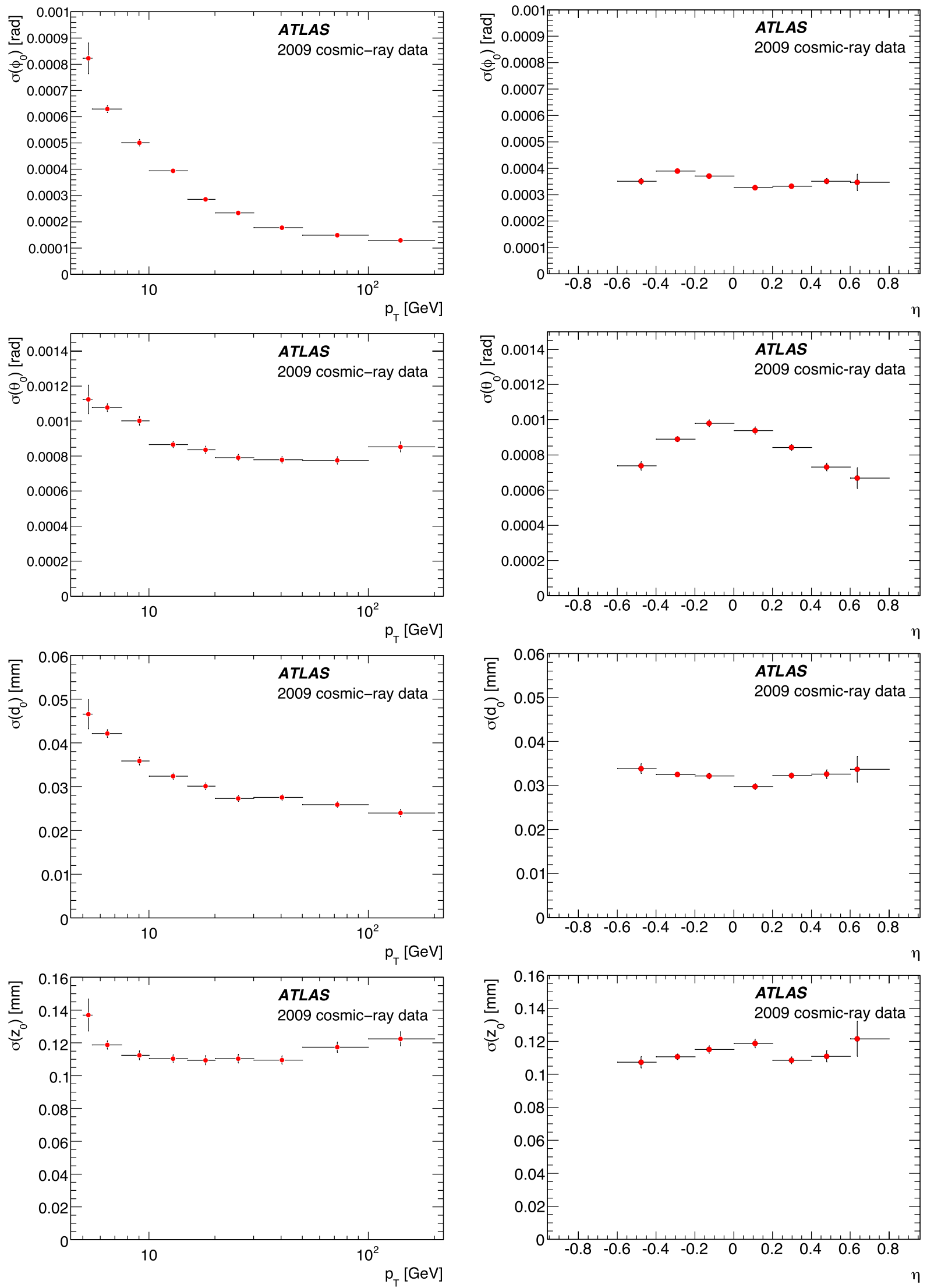

Fig. 6 Resolution on track parameters $\phi_{0}, \theta_{0}, d_{0}$ and $z_{0}$, obtained from split tracks, as a function of $p_{\mathrm{T}}$ (left column) and $\eta$ (right column) 
in the track parameters obtained from the two split tracks, $\Delta \lambda=\lambda_{\text {up }}-\lambda_{\text {low }}$. Each such distribution has an expectation value of 0 and a variance equal to two times the square of the parameter resolution: $\operatorname{var}(\Delta \lambda)=2 \sigma^{2}(\lambda)$. For each parameter, the mean and resolution of this difference distribution have been studied in bins of $p_{\mathrm{T}}$ and $\eta$. Since the cosmic-ray muon momentum distribution is a steeply falling function, the $p_{\mathrm{T}}$ value for each bin is taken as the mean of the $p_{\mathrm{T}}$ distribution in that bin. For the resolutions, the results are shown in Fig. 6. The absence of data points in the range of $-0.8<\eta<-0.6$ is due to a requirement that there be at least 50 muons per bin. The means are roughly independent of $p_{\mathrm{T}}$ and $\eta$ and show no significant bias, with the exception of the $z_{0}$ distribution. That shows a small bias that varies with $\eta$, but with a magnitude that is less than about $60 \mu \mathrm{m}$ over the $\eta$-range investigated. This is negligible relative to the MS-ID bias already discussed. The means and resolutions obtained from tracks with $p_{\mathrm{T}}>30 \mathrm{GeV}$ are shown in Table 1.

A similar study of the track momentum reconstructed in the upper and lower halves of the detector shows that the mean of the momentum-difference distribution $\left(p_{\text {up }}-p_{\text {low }}\right)$ is consistent with zero and flat as a function of $p_{\mathrm{T}}$ and $\eta$. For studies of the $p_{\mathrm{T}}$ resolution, slightly looser cuts are employed in order to increase the statistics, particularly in the high-momentum region. For tracks having momenta above $50 \mathrm{GeV}$ the requirement of a pixel hit is removed and the cuts on $\left|d_{0}\right|$ and $\left|z_{0}\right|$ are loosened to $1000 \mathrm{~mm}$. Figure 7 shows the relative $p_{\mathrm{T}}$ resolution for ID, MS and CM tracks as a function of $p_{\mathrm{T}}$. For each pair of upper/lower tracks, the value of the transverse momentum was evaluated at the perigee. The difference between the values obtained from the upper and lower parts of the detector, divided by their average

$$
\frac{\Delta p_{\mathrm{T}}}{p_{\mathrm{T}}}=2 \frac{p_{\text {Tup }}-p_{\text {Tdown }}}{p_{\text {Tup }}+p_{\text {Tdown }}}
$$

was measured and plotted in eleven bins of $p_{\mathrm{T}}$. As above, the plotted $p_{\mathrm{T}}$ value is the mean of the $p_{\mathrm{T}}$ distribution in that bin. The results of this procedure have been fitted to

Table 1 Overview of the track parameter bias and resolution for CM tracks obtained with the track-splitting method for 2009 cosmic-ray data, for tracks with $p_{\mathrm{T}}>30 \mathrm{GeV}$

\begin{tabular}{lll}
\hline Parameter & Mean & Resolution \\
\hline$\phi_{0}(\mathrm{mrad})$ & $-0.053 \pm 0.005$ & $0.164 \pm 0.004$ \\
$\theta_{0}(\mathrm{mrad})$ & $0.27 \pm 0.03$ & $0.80 \pm 0.02$ \\
$d_{0}(\mu \mathrm{m})$ & $-0.9 \pm 0.7$ & $26.8 \pm 0.8$ \\
$z_{0}(\mu \mathrm{m})$ & $2.0 \pm 3.7$ & $116.6 \pm 2.9$ \\
\hline
\end{tabular}

parametrizations appropriate to each particular track class. For the ID the fit function was:

$$
\frac{\sigma_{p_{\mathrm{T}}}}{p_{\mathrm{T}}}=P_{1} \oplus P_{2} \times p_{\mathrm{T}},
$$

where $P_{1}$ is related to the multiple scattering term and $P_{2}$ to the ID intrinsic resolution. For the MS tracks, the same function is used but with an additional term (coefficient $P_{0}$ ) related to uncertainties on the energy loss corrections associated with the extrapolation of the MS track parameters to the perigee:

$$
\frac{\sigma_{p_{\mathrm{T}}}}{p_{\mathrm{T}}}=\frac{P_{0}}{p_{\mathrm{T}}} \oplus P_{1} \oplus P_{2} \times p_{\mathrm{T}} .
$$

For the combined resolution a more complex function is needed:

$$
\frac{\sigma_{p_{\mathrm{T}}}}{p_{\mathrm{T}}}=P_{1} \oplus \frac{P_{0} \times p_{\mathrm{T}}}{\sqrt{1+\left(P_{3} \times p_{\mathrm{T}}\right)^{2}}} \oplus P_{2} \times p_{\mathrm{T}},
$$

where $P_{1}$ is related to the multiple scattering term, $P_{2}$ to the intrinsic resolution at very high momentum and the $P_{3}$ term describes the intermediate region where ID and MS resolutions are comparable.

Table 2 compares the fitted sizes of the multiple scattering and intrinsic resolution terms for the ID, MS and CM tracks. For the CM tracks the multiple scattering term is determined mainly by the ID contribution while the intrinsic high-energy resolution comes mainly from the MS measurement.

Extrapolation of the fit result yields an ID momentum resolution of about $1.6 \%$ at low momenta and of about $50 \%$ at $1 \mathrm{TeV}$. The MS standalone results are improved over those previously obtained [9]: the resolution extrapolated to $1 \mathrm{TeV}$ is about $20 \%$. As expected the ID and MS systems dominate the resolution at low and high $p_{\mathrm{T}}$, respectively. However, at intermediate momenta from about 50 to $150 \mathrm{GeV}$ both systems are required for the best resolution. The $\pm 1 \sigma$ region returned by the fit to the resolution for the CM tracks is shown as the shaded region in Fig. 7.

\subsection{Muon energy loss in the ATLAS calorimeters}

Muons traverse more than 100 radiation lengths between the two tracking systems. Interactions with the calorime-

Table 2 Fitted values of the multiple scattering and intrinsic momentum resolution terms (as described in the text) for ID, MS and CM tracks

\begin{tabular}{lll}
\hline Fitted Resolution & $P_{1}$ & $P_{2}$ \\
\hline Inner Detector & $1.6 \pm 0.1 \%$ & $(53 \pm 2) \times 10^{-5} \mathrm{GeV}^{-1}$ \\
Muon Spectrometer & $3.8 \pm 0.1 \%$ & $(20 \pm 3) \times 10^{-5} \mathrm{GeV}^{-1}$ \\
Combined Muon & $1.6 \pm 0.1 \%$ & $(23 \pm 3) \times 10^{-5} \mathrm{GeV}^{-1}$ \\
\hline
\end{tabular}




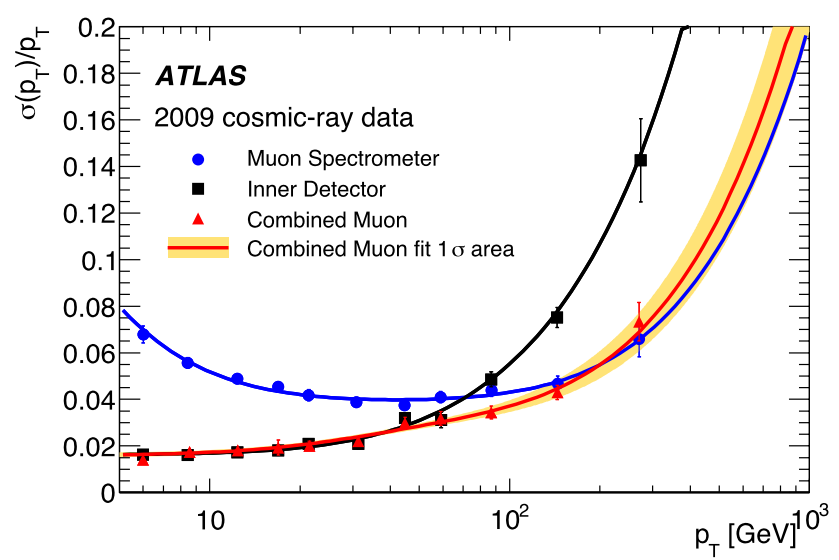

Fig. 7 Resolution on relative $p_{\mathrm{T}}$ as a function of $p_{\mathrm{T}}$ for ID and MS standalone tracks, and for CM tracks. The shaded region shows the $\pm 1 \sigma$ region of the fit to the resolution curve for the CM tracks

ter material result in energy losses. These losses are typically around $3 \mathrm{GeV}$, mainly due to ionization, but are subject to fluctuations, especially for high momentum muons which can deposit a large fraction of their energy via bremsstrahlung. Muon reconstruction in collision events depends on a correct accounting for these losses, as does determination of the missing transverse energy in the event. A parametrization of these losses is normally used for extrapolating the track parameters measured by the MS to the perigee where they are defined. However, since $80 \%$ of the material between the trackers is instrumented by the calorimeters, studies of the associated energy deposits in the calorimeter should allow improvements to the resolution in the case of large losses.

This possibility has been investigated using cosmic-ray muons traversing the barrel part of ATLAS. The analysis is based on the PCM sample from a single 2009 cosmicray run, consisting of about one million events. Strict criteria were applied to ensure pseudo-projective trajectories that are well measured in the relevant tracking subsystems: the SCT and the TRT in the Inner Detector, and the MDT and RPC systems in the Muon Spectrometer. The analysis was restricted to tracks crossing the bottom part of the Tile Calorimeter, in the region $|\eta|<0.65$. A track-based algorithm [19-21] was used to collect the muon energy deposits in the calorimeters. The trajectory of the particle was followed using the ATLAS extrapolator [22], which, using the ATLAS tracking geometry [23], takes into account the magnetic field, as well as material effects, to define the position at which the muon crossed each calorimeter layer. The cells within a predefined 'core' region around these points were used for the measurement of the energy loss. This region was optimized according to the granularity and the geometry of each calorimeter layer. Only cells with $|E|>2 \sigma_{\text {noise }}$ were considered. Here $\sigma_{\text {noise }}$ is the electronics noise for the channel and $|E|$ is used instead of $E$ to avoid biases. As a check that this procedure properly reconstructs the muon energy deposits, the total transverse energies reconstructed in calorimeter cells within cones of $\Delta R=\sqrt{(\Delta \eta)^{2}+(\Delta \phi)^{2}}$ of $0.2,0.3$ and 0.4 , around the particle trajectory, were determined. From these, the sum of the transverse energy inside the core region $\left(E_{\mathrm{T}}^{\text {core }}\right)$ was subtracted. In collision events these quantities can be used to define the muon isolation, while in this analysis they indicate how much energy is deposited outside the core. The distributions of these quantities, shown in the top plot of Fig. 8, are reasonably centered around zero with widths that increase with the cone size, as expected due to the inclusion of a larger number of cells. Small energy losses outside the $E_{\mathrm{T}}^{\text {core }}$ region shift the distributions to slightly positive values, due to either uncertainties in the extrapolation process or to radiative losses.

As a measure of the energy deposited by the muon, $E_{\mathrm{T}}^{\text {core }}$ is used with no additional correction. Monte Carlo simulations of single muons in the barrel region show that this method provides a nearly unbiased energy determination, with $2 \%$ scale uncertainty and $11 \%$ resolution for the energy deposited by $100 \mathrm{GeV}$ muons. To allow comparison of these losses with the difference between the momenta reconstructed in the two tracking systems, a parametrization of the losses in the dead (uninstrumented) material, $E_{\text {dead }}$, is added to the calorimeter measurement. The tracking geometry provides this information in combination with the extrapolator. The energy measured in the calorimeter, corrected for the dead material, is compared with the momentum difference between Inner Detector and Muon Spectrometer tracks in the middle plot of Fig. 8. The mean values of the momentum-difference and energy-sum distributions are $3.043 \mathrm{GeV}$ and $3.044 \mathrm{GeV}$ respectively. The typical momentum of the selected tracks is 16 (13) GeV in the Inner Detector (Muon Spectrometer), measured (see Fig. 7) with a resolution of about $2 \%(4 \%)$, while the energy collected in the calorimeters, $E_{\text {calo }}$, is on average $2.4 \mathrm{GeV}$, with a precision of about $10-20 \%$. The RMS values of the two distributions are $1.081 \mathrm{GeV}$ and $0.850 \mathrm{GeV}$ respectively. In simulation the two distributions have means of $3.10 \mathrm{GeV}$ and $3.12 \mathrm{GeV}$ compared to a true energy loss distribution with a mean of $3.11 \mathrm{GeV}$ and an RMS of $0.750 \mathrm{GeV}$. The resolutions were $0.950 \mathrm{GeV}$ and $0.820 \mathrm{GeV}$, respectively, roughly consistent with the measured values. The bottom plot in Fig. 8 shows the distribution of $\left(P_{\mathrm{ID}}-P_{\mathrm{MS}}\right)-\left(E_{\mathrm{calo}}+E_{\text {dead }}\right)$, which has a mean of $-0.012 \mathrm{GeV}$ and an RMS of $1.4 \mathrm{GeV}$. This distribution is dominated by contributions from rather lowmomentum tracks. Restricting to the momentum region of $10-25 \mathrm{GeV}$ retains about $40 \%$ of the statistics and yields a distribution with mean and RMS of $-0.004 \mathrm{GeV}$ and $1.0 \mathrm{GeV}$, respectively.

Although the tracking systems are relatively more precise than the calorimeters, in both data and Monte Carlo simulation, the RMS of the energy-sum distribution from the 

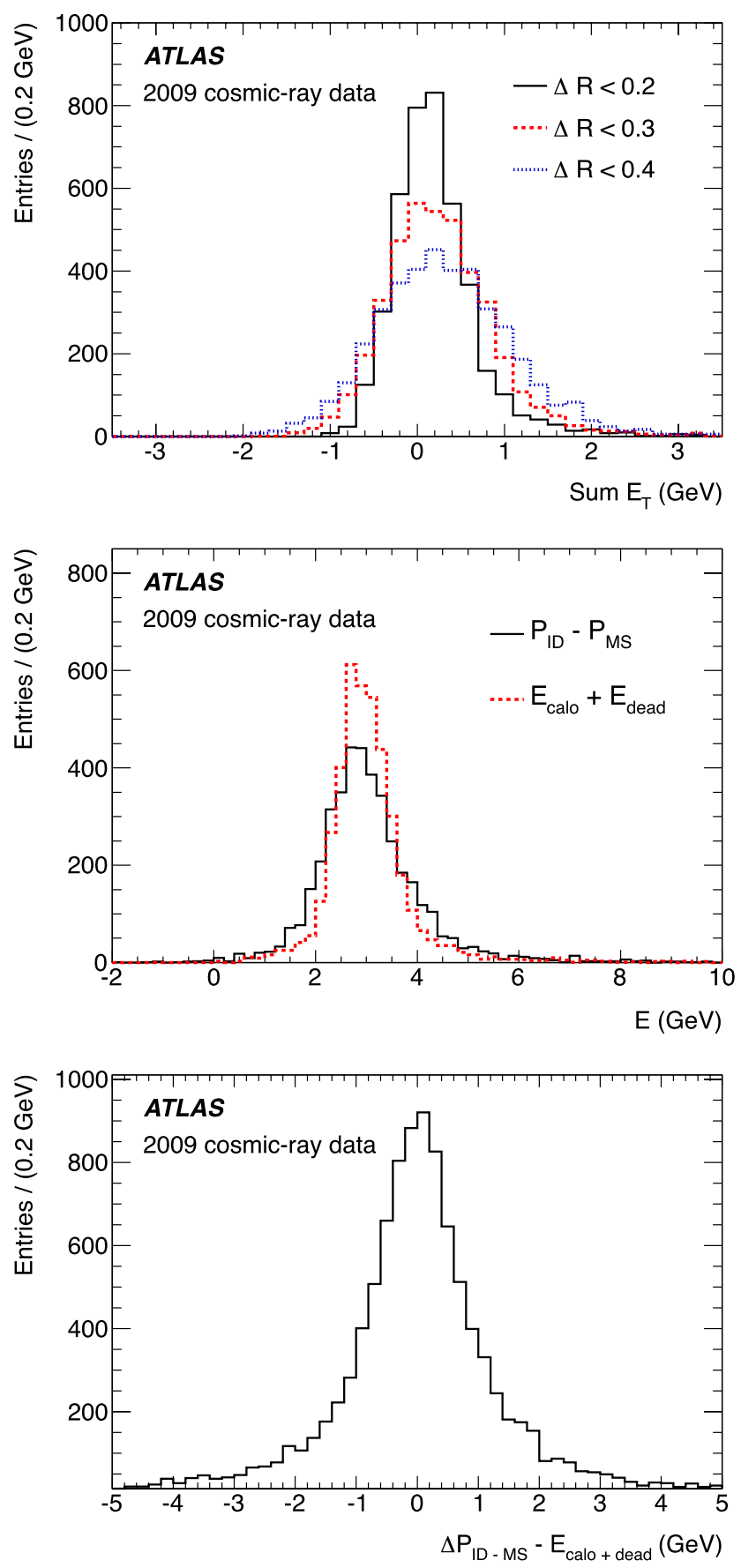

Fig. 8 The upper plot shows the sum of the transverse energy around muon tracks, outside the core region, for cones of $\Delta R=0.2,0.3$ and 0.4. The middle plot compares the momentum difference between Inner Detector and Muon Spectrometer tracks $\left(P_{\mathrm{ID}}-P_{\mathrm{MS}}\right)$ with the sum of the energy loss measured in the calorimeters, $E_{\text {calo, }}$, and the parametrized energy loss in the inert material, $E_{\text {dead }}$. The lower plot shows the distribution of $\left(P_{\mathrm{ID}}-P_{\mathrm{MS}}\right)-\left(E_{\text {calo }}+E_{\text {dead }}\right)$

calorimeter is smaller than that of the momentum-difference distribution from the tracking systems. Use of the calorimeter information may therefore allow future improvements to the combined tracking for muons.

\subsection{Identification of electrons}

The identification of electrons is performed by algorithms relying on information from both the EM calorimeter and the ID. Two methods are used, one seeded by tracks and the other by EM calorimeter clusters. The cluster-based algorithm is the standard identification tool, with clusters seeded using a sliding-window algorithm [24]. This algorithm, used only for the identification of electromagnetic $(e / \gamma)$ objects in the EM calorimeter, uses a fixed grid of calorimeter cells in $\eta \times \phi$, centered on a seed cell having a signal-to-noise ratio exceeding a set threshold. For a cluster to form an electron candidate, there must normally be an ID track nearby in $\eta$ and $\phi$. However, in cosmic-ray events many tracks have only barrel TRT $(r-\phi)$ hit information, in which case the association is done only in $\phi$. The threshold for the reconstruction of an $e / \gamma$ object with the standard selection is about $3 \mathrm{GeV}$. To improve the identification of electrons with lower $p_{\mathrm{T}}$, a track-seeded algorithm is employed. This first searches for tracks in the ID with $p_{\mathrm{T}}>2 \mathrm{GeV}$ and hits in both the SCT and Pixel Detectors. These tracks are extrapolated to the second layer of the EM calorimeter and a $3 \times 7$ $(\eta \times \phi)$ cell cluster is formed about this point; the cell size in this layer varies with $\eta$ but is $0.025 \times 0.025$ in $\eta \times \phi$ over the acceptance for this analysis. In both algorithms, the track momentum and the energy of the associated calorimeter cluster are required to satisfy $E / p<10$. This section describes the use of these standard techniques for the selection of a sample of $\delta$-electrons, which are used to investigate the calorimeter response to electrons with energies in the $5 \mathrm{GeV}$ range. Section 4.4 will describe an alternative low- $p_{\mathrm{T}}$ selection which can identify electrons down to $p_{\mathrm{T}}$ of about $500 \mathrm{MeV}$, using a more sophisticated clustering algorithm for determination of the energy of the associated electromagnetic calorimeter cluster.

Electron identification relies in part on the particle identification abilities of the TRT. Transition radiation (TR) is produced by a charged particle crossing the boundary between two materials having different dielectric constants. The probability of producing TR photons depends on the Lorentz factor $(\gamma=E / m)$ of the particle. The effect commences at $\gamma$ factors around 1000 which makes it particularly useful for electron identification, since this value is reached for electrons with energies above about $500 \mathrm{MeV}$. For muons, these large $\gamma$ factors occur only for energies above about $100 \mathrm{GeV}$. The TR photons are detected by absorption in the chamber gas which is a xenon mixture characterized by a short absorption length for photons in the relevant energy range. The absorption leads to high electronic pulses; pulses due to energy deposits from particles which do not produce transition radiation are normally much lower. A distinction between the two classes of particles can therefore be made by comparing the pulse heights against 
high and low thresholds, and looking at the fraction of highthreshold hits for a given track. This fraction is referred to below as the TR ratio.

The production of electrons in cosmic-ray events is expected to be dominated by knock-on or $\delta$-electrons produced by ionization caused by cosmic-ray muons. The energy distribution of such electrons is typically rather soft, but has a tail extending out into the $\mathrm{GeV}$ region, where the standard electron identification tools can be employed. The experimental signature of such an event consists of a muon track traversing the muon chambers at the top and bottom of the detector, having corresponding MIP-like energy deposits in the calorimeters, accompanied by a second lower-momentum track in the ID associated with a cluster in the EM calorimeter, as illustrated by the event displayed in Fig. 9. In the upper view, the incoming and outgoing muon tracks, are seen to leave hits in three muon layers on the top of ATLAS and in two layers below, as well as in the Inner Detector. In the lower, expanded view of the ID region the muon track and the electron candidate track are shown with the associated hits in the silicon detectors as well and those in the TRT, which are illustrated by either light or dark markers, depending on whether they are low- or high-threshold. The candidate electron track clearly displays a larger number of high-threshold TRT hits, as expected for an electron, as well as an association to a cluster of energy in calorimeter (at the bottom). Other low-energy deposits in the calorimeter have been suppressed.

The search was performed using data from the PCM sample obtained from cosmic-ray running in the fall of 2008 . Based on the expected topology, events were selected if they satisfied the following requirements:

- 2 or more ID tracks.

- 1 electron in the bottom of the detector (since the muons come from above).

- 1 or more muon tracks: if there is more than one there must be at least one track in the top and bottom halves of the detector, consistent with coming from a muon of the same charge.

The events so selected are referred to below as the signal sample, or the ionization sample.

There is one important background for which this selection can lead to the identification of fake electron candidates. A highly energetic muon can emit a bremsstrahlung photon that does not convert within the ID. This photon will produce a cluster in the EM Calorimeter that can be incorrectly associated with the muon track if the track and cluster are nearby, creating a fake electron candidate. The signature for this process is one incoming and one outgoing muon track in the MS, one track in the ID and a cluster in the lower part of the EM Calorimeter. This signature can be clearly distinguished from the true electron production processes by the
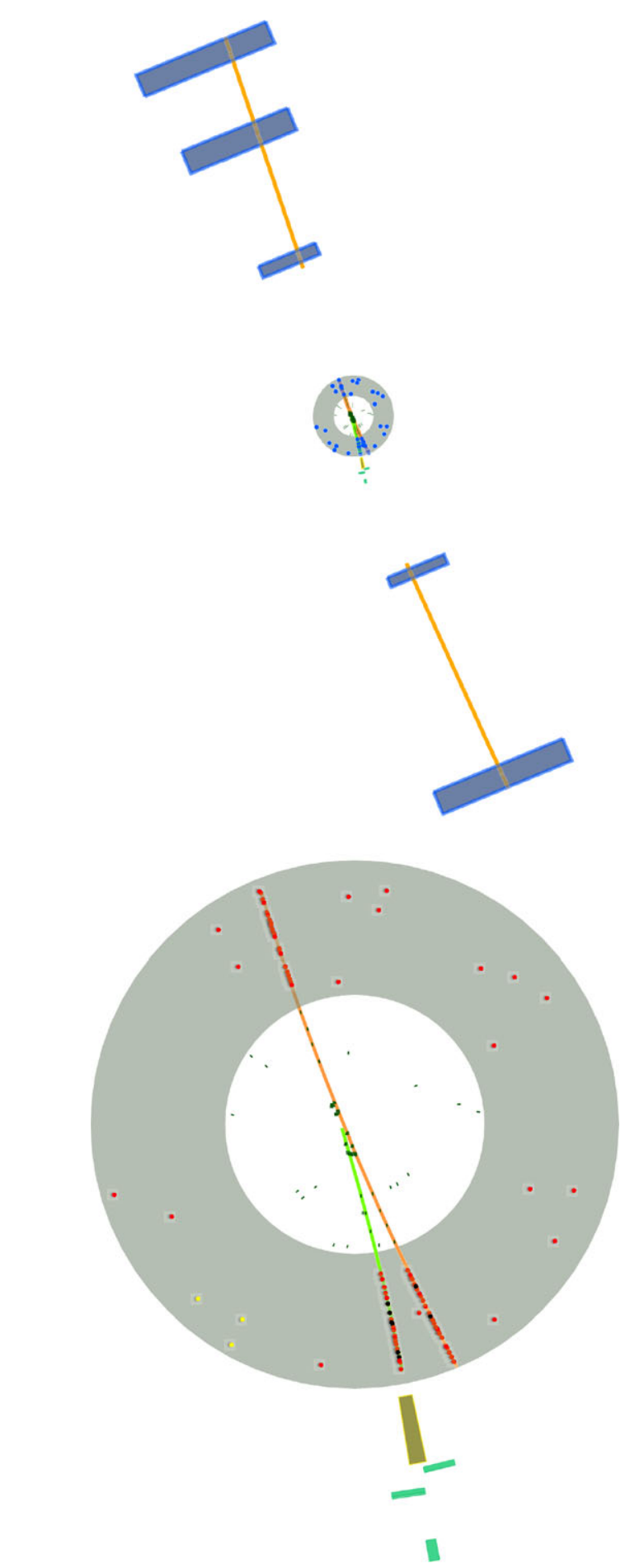

Fig. 9 Event display of a typical $\delta$-electron candidate event. The upper figure shows a view that includes the three layers of muon detectors on either side, while the lower plot shows a close-up view of the Inner Detector. The shaded region represents the volume of the TRT, while the inner region is occupied by the SCT and Pixel detectors. The two ID tracks, and associated hits, are clearly visible. High- and low-threshold TRT hits are displayed with the dark and light markers, respectively. The calorimeter cluster associated with the electron candidate is also shown. Other low-energy deposits in the calorimeter are suppressed 
number of tracks in the ID (except for muon decays in flight which are expected to contribute only a very small fraction of the electrons of interest in this analysis). Nevertheless, for muon bremsstrahlung events, an additional track may be present (due for example to an overlapping cosmic ray event or a correlated cosmic-ray muon due to an air shower event) leading to an event with the same signature as the signal process. This background source should produce equal numbers of electron and positron candidates in contrast to true $\delta$-electrons events, where only negatively charged electrons are produced. To study this background (in which electron candidates are actually muons), a sample of events depleted in $\delta$-electrons and enriched muon bremsstrahlung events, was selected using the requirements:

- exactly 1 ID track

- 1 electron in the bottom of the detector

- 1 or more muon tracks

In the analysis of the signal and background samples, slightly modified versions of standard algorithms were used to identify electrons. The standard selection [1] defines three classes of candidates: loose, medium and tight, according to increasingly stringent cuts on the typical properties of electron tracks and their associated EM showers, particularly quantities related to the longitudinal and transverse shower development. For the analysis discussed here, a "modified medium" selection is adopted, which is a combination of selection criteria applied in the standard medium and tight selections, with slight modifications to allow for the different topology of the cosmic-ray muon events. In particular, since most of the muons do not pass through the SCT or Pixel Detector, requirements on the number of hits in the silicon detectors are replaced with quality cuts based on the number of TRT barrel hits and the $\phi$ matching of the electron track to the EM cluster. A cut on $\left|z_{0}\right|$ is made to ensure that tracks are in the barrel part of the TRT.

In addition to this modified medium selection, a tight selection is defined by two additional requirements:

$-0.8<E / p<2.5$

- TR ratio $>0.08$

Note that both of these cuts are actually slightly $\eta$-dependent, following the standard tight selection. The values quoted above are those applied over most of the acceptance. After application of the modified medium selection, there are 81 events in the signal sample and 1147 in the background sample. Since the background candidates arise dominantly from the case where the EM cluster is associated to the cosmicray muon, this sample can be used to model the properties of the corresponding background events in the signal sample, in which the requirement of an additional ID track greatly reduces the number of events. Because $E / p$ and the TR ratio are correlated, these quantities are shown plotted against one another in the upper plots of Fig. 10, separately for the signal and background samples. The open and solid markers together show the distribution of candidates passing the modified medium selection. The solid markers show the candidates that also survive the tight selection. In each plot, the dotted lines show the cuts applied (as quoted above) on each quantity, for the majority of the candidates. These define the signal region which is enclosed by the overlaid solid lines. The open markers in the signal region and solid markers in the background region arise due to the slight $\eta$-dependence of the cuts. There are 34 events from the signal sample passing all cuts, compared to 13 from the background sample. Of the 34 events in the signal region, 4 are positively charged.

The sample of 34 candidates was investigated further in order to confirm the identification of these as electrons and to determine the number of $\delta$-electrons by estimating the background in the signal sample. This was done by performing a three-parameter, binned maximum-likelihood fit to the two-dimensional TR ratio vs. $E / p$ distribution for the background sample and then fitting the resulting background shape to the ionization sample in the regions outside the signal acceptance. The results of this procedure are displayed in the lower plots of Fig. 10. Note that the fit uses finer binning than is used for these projections. The plot on the left shows the distribution of the TR ratio for the 81 candidates passing the modified medium cuts (points with error bars) while the dashed histogram shows the 47 events in the background region and the solid curve shows the projection of the two-dimensional binned maximum likelihood fit, which provides a good description of the distribution from candidates in the background region (dashed histogram). The right-hand plot shows the distribution of $E / p$ for all candidates remaining after the additional application of the tightselection cut on the TR ratio. The solid curve again shows the projection of the two-dimensional background fit leading to an estimate for the background contribution in the signal region (indicated by the dotted vertical lines) of $(8.3 \pm 3.0)$ events. This is consistent with the hypothesis that the dominant background is muon bremsstrahlung, which should produce equal numbers of positive and negative candidates, and the observation of 4 positively charged candidates in the signal sample.

As a final check on the candidate events, several distributions related to shower profiles were compared to expectations based on a Monte Carlo simulation of projective electrons (produced at the nominal IP) with transverse energy of $5 \mathrm{GeV}$, in the region $|\eta|<0.8$ which is appropriate for comparison with the cosmic-ray electron sample obtained with this selection. These comparisons are shown in Fig. 11. The upper left plot shows the lateral containment, in the $\phi$ direction, of energy in the cells of the second layer of the EM calorimeter, as defined by the ratio $E_{3 \times 3} / E_{3 \times 7}$ where $E_{i \times j}$ represents the energy deposited in a collection of cells 

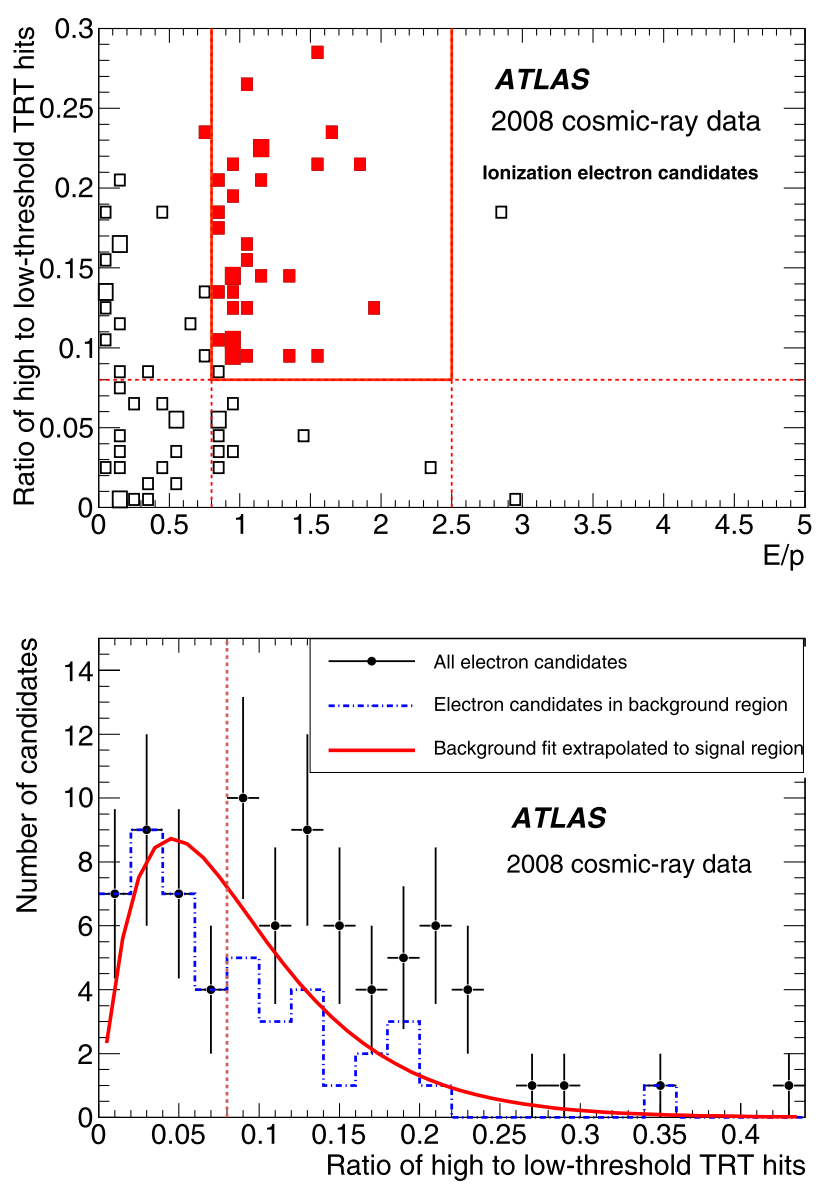

Fig. 10 The upper plots show the two-dimensional distributions of the TR ratio vs. $E / p$ for the ionization sample (left) and the background sample (right). The open and solid markers together show the distribution of electron candidates passing the modified medium cuts. The solid markers indicate the candidates which also survive the tight selection. The dotted lines show the cuts applied to most of the events having $\eta \approx 0$ and low transverse energy: $0.8<E / p<2.5$ and TR ratio $>0.08$. The solid lines indicate the corresponding signal region. Two outliers at high TR ratio (1 in signal, 1 in background region), and two outliers at high $E / p$ are not shown. The lower plots show projections of the fit result for the ionization sample. The left plot shows the distribution of the TR ratio for all 81 electron and positron candidates

of size $i \times j$ in $\eta \times \phi$. A large mean value is observed for both data and Monte Carlo, as expected since electrons tend to have a small lateral shower width. The upper right plot shows the lateral extent of the shower in $\eta$, in the first layer of the EM calorimeter, as measured by the sum of the cellcluster $\eta$ separations, weighted by the cell energy. This also shows good agreement between data and Monte Carlo. The other quantities plotted are related to the longitudinal shower shape: the lower left plot shows the fraction of the total cluster energy deposited in the first layer of the EM calorimeter while the lower right plot shows the fraction of energy in the second layer. In both cases the average value should be about $40 \%$ for electrons, as these tend to start showering early in the calorimeter. There is reasonable agreement between
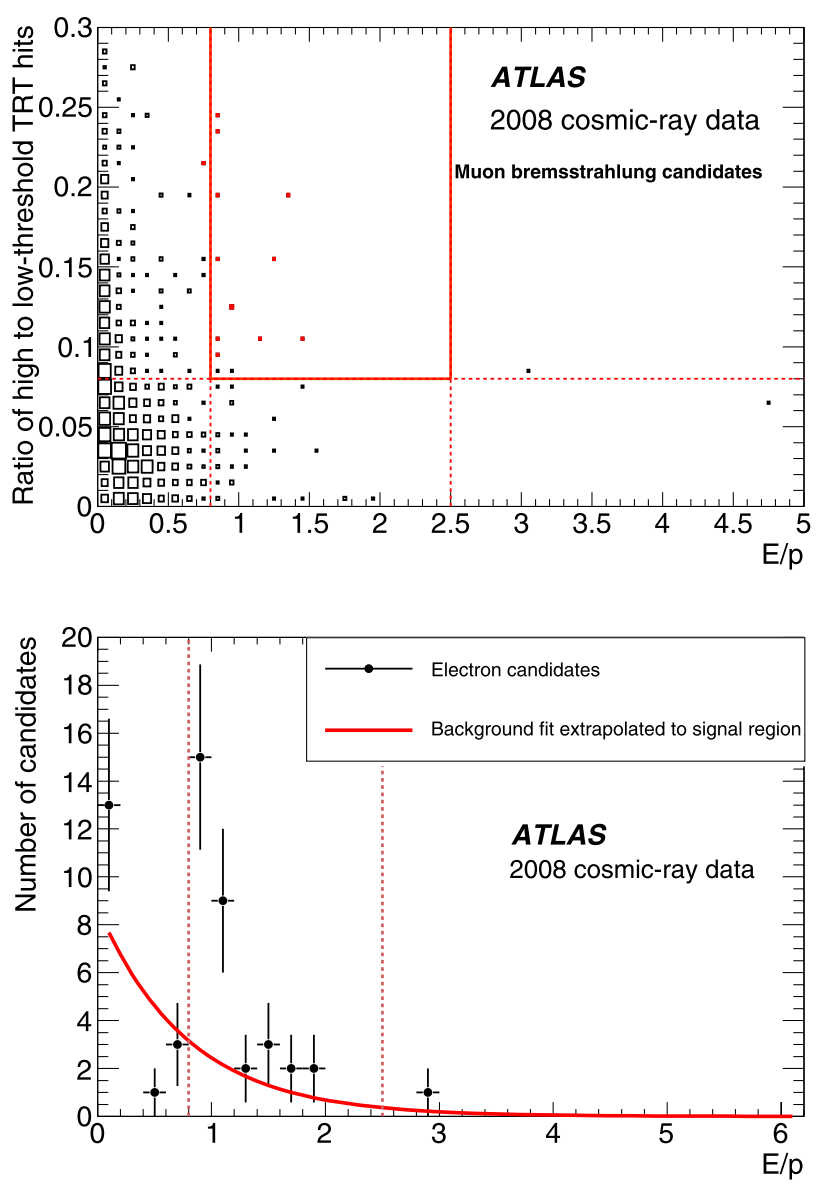

after the modified medium cuts (points with error bars). The dashed histogram shows the 47 events in the background region and the curve shows the projection of the two-dimensional binned maximum likelihood fit. The dotted vertical line indicates the lower selection cut applied to the bulk of the events. The right plot shows the distribution of $E / p$ for all modified medium electron candidates after the additional application of the tight-selection cut on the TR ratio. The curve shows the projection of the two-dimensional background fit from which the number of background events under the signal region is estimated. The dotted vertical lines represent the upper and lower selection cuts on $E / p$, applied to the bulk of the data

data and Monte Carlo, but both show some small discrepancies. These arise from the fact that several of the data events have much larger energies than were used for the Monte Carlo sample, which consists entirely of electrons with a transverse energy of $5 \mathrm{GeV}$. The deviations are consistent with what would be expected from the bremsstrahlung background in the sample. Those events can be of higher energy than the electron events, affecting the energy distributions of the showers, particularly the longitudinal energy profiles. Distributions of the fractions of energy deposited in the presampler and in layer 3 of the EM calorimeter show a similar level of agreement with the distributions from the projectiveelectron Monte Carlo sample. 

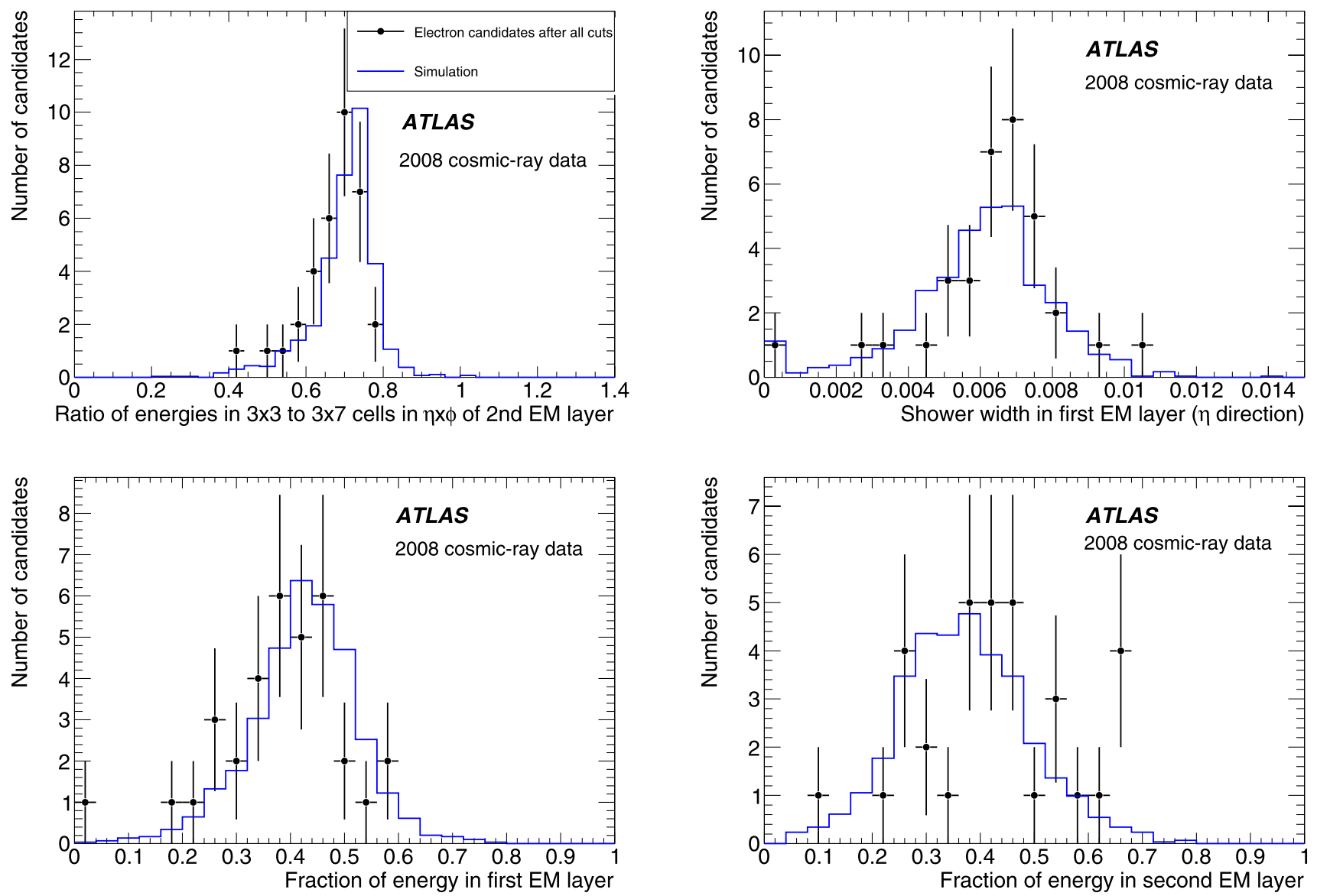

Fig. 11 Comparison of shower profiles for all $34 \mathrm{e}^{ \pm}$candidates to those from simulated projective electrons with a transverse energy of $5 \mathrm{GeV}$ and $|\eta|<0.8$. The data points indicate the electrons from the cosmic-ray data, while the histograms indicate distributions obtained from the simulated electrons. The upper left plot shows the ratio of energies in $3 \times 3$ over $3 \times 7$ cells in $\eta \times \phi$ in the second layer of the EM

\subsection{Identification of low momentum electrons}

The majority of the electrons in the cosmic-ray data are expected to be of low energy, of the order of a few hundred $\mathrm{MeV}$. The probability of producing an electron with sufficiently high momentum to produce a standard $\mathrm{e} / \gamma$ cluster in the EM calorimeter is rather small, as reflected in the relatively low statistics available using the selection described in the previous section.

In addition to the sliding-window cluster used for the standard electron identification, ATLAS employs a topological clustering algorithm [25] which groups adjacent cells with energies above certain thresholds into clusters which are thus composed of varying number of cells, providing for better noise suppression. Each topological cluster is seeded by a cell having a signal-to-noise ratio $\left(|E| / \sigma_{\text {noise }}\right)$ above a threshold $t_{\text {seed }}$, and is then expanded by iteratively adding neighboring cells having $|E| / \sigma_{\text {noise }}>t_{\text {neighbor. Following }}$ the iterative step, the cluster is completed by adding all

calorimeter. The upper right plot shows the energy-weighted shower width in $\eta$, in the first layer of the EM calorimeter. The lower left (right) plot shows the distribution of the fraction of energy in the first (second) layer of the EM calorimeter. The Monte Carlo distributions are normalized to the number of data events

direct neighbor cells along the perimeter having signal-tonoise above $|E| / \sigma_{\text {noise }}>t_{\text {cell }}$. Several types of topological clusters (differing in $t_{\text {seed }}, t_{\text {neighbor }}$ and $t_{\text {cell }}$ ) are used by ATLAS, for the reconstruction of calorimeter energy deposits from hadrons, electrons and photons, over the full range of $\eta$.

A selection based on the matching of an ID track to an EM topological cluster was applied to the cosmic-ray data. This analysis, run on data from both the 2008 and 2009 cosmic-ray data-taking periods, is similar to the one described in the previous section, also focusing on events in the barrel part of the detector. The topological signature of the electron events is the same as described in Sect. 4.3 and the data sample is separated into signal and background samples in a similar way, based on the number of tracks; electrons are again searched for in events with at least 2 ID tracks, while events with only one reconstructed track are used as a background sample. Candidate tracks must match an EM cluster from the topological clustering algo- 
rithm with $t_{\text {seed }}=4, t_{\text {neighbor }}=3$ and $t_{\text {cell }}=0$. This allows the reconstruction of electromagnetic clusters with energies down to about $500 \mathrm{MeV}$.

Electron candidate tracks are required to be in the barrel region of the TRT and to have at least 25 TRT hits to ensure good quality tracks. There is no requirement of silicon hits. The TR ratio is required to exceed 0.1. Further suppression of backgrounds is achieved using various moments of the calorimeter cluster designed to select the compact clusters typical of electromagnetic objects. For example, Fig. 12 shows data and Monte Carlo distributions for the topological cluster moment $\lambda_{\text {center }}$, defined as the distance from the calorimeter front face to the shower center, along the shower axis. The two plots show distributions for signal and background events accepted by the low- $p_{\mathrm{T}}$ electron selection, before (left) and after (right) application of the cluster-moment-based selection criteria. The left-hand plot shows the distribution obtained with the signal selection applied to the cosmic-ray data and Monte Carlo along with the expected distribution for true electrons from the Monte Carlo. The MC distribution has been normalized to the data. The cut of $\lambda_{\text {center }}<220 \mathrm{~mm}$ is indicated by the dotted vertical line. Muons which traverse the calorimeter as MIPs leave their energy uniformly distributed in depth, producing a peak in the distribution at the point which corresponds to half the depth of the EM calorimeter. The righthand plot shows the selected region after all cuts, for the signal events, the events from the background sample, and for those events from the Monte Carlo which are matched to real ("Monte Carlo truth") electrons. Good agreement is observed between data and Monte Carlo.

As in the electron analysis described in the previous section, signal and background regions are defined in the plane of the TR ratio vs. $E / p$. A fit is performed to the data in the background region of the background sample and then used to estimate the background in the signal sample. Selected events from both samples are shown in Fig. 13 for data and Monte Carlo. The Monte Carlo plots also include the distributions of electron candidates that are matched to Monte Carlo truth electrons, corresponding to $97 \%$ of the candidates selected from that sample. The upper plots show the $E / p$ distributions for the selected events. The final selection cut of $E / p>0.5$ is illustrated by the dashed line. This lower $E / p$ cut, relative to the analysis described in Sect. 4.3, is needed as the lower $p_{\mathrm{T}}$ electrons suffer relatively more energy loss in the detector material before reaching the calorimeter. The lower plots show the momentum distributions of the electron candidates passing the full selection, and show acceptance down to $\sim 500 \mathrm{MeV}$.

In general, ATLAS does not attempt to identify electrons down to such low energies. This commissioning analysis is intended to illustrate the flexibility that exists for the identification of electrons. While the topological clustering tech- nique discussed here is not part of the standard electron identification algorithm for most of the detector acceptance, it is the default technique in the forward region $(2.5<|\eta|<4.9)$. This region is beyond the tracking acceptance, so in that case no matching is done to tracks. Instead, electrons are identified by topological clusters having properties (e.g. cluster moments) that are typical of electromagnetic energy deposits.

\subsection{Commissioning of the $\tau$ reconstruction and identification algorithms}

As discussed earlier, the cosmic-ray data have also been used to examine the tools used for the identification of $\tau$ leptons. A leptonically decaying $\tau$, where the visible final state is either an electron or muon, is difficult to distinguish from a primary electron or muon. The $\tau$ identification algorithm therefore focuses on hadronically decaying $\tau$ leptons, for which the dominant final states consists of either one or three charged hadrons and some number of neutrals. Reconstruction of these final states typically involves several subdetectors: one expects ID tracks associated with the charged hadrons and energy deposits in the calorimeter, from both charged and neutral hadrons. The neutrals are dominantly pions which decay to two photons and leave their energy in the EM calorimeter. Hadronically-decaying $\tau$ leptons are often referred to as $\tau$-jets.

The identification of $\tau$ leptons is primarily concerned with distinguishing these from a large background due to QCD jets. The identification algorithm relies upon features such as the track multiplicity, which should be low for $\tau$ leptons, and the transverse profile of the energy deposits in the detector, which is typically narrower for $\tau$-jets than for those from QCD. A $\tau$ will almost always have a final state with either one or three tracks, though some allowance is made for imperfect track reconstruction in the ID. Finally, the $\tau$ final state will often result in a prominent deposit in the electromagnetic calorimeter, associated with photons produced by the decays of neutral pions.

The identification of $\tau$ leptons is performed by an algorithm that can be seeded either by a track from the ID or by an energetic jet in the calorimeter. Track-based $\tau$ candidates are seeded by one good quality track having $p_{\mathrm{T}}>6 \mathrm{GeV}$ and can incorporate up to seven additional tracks with $p_{\mathrm{T}}>$ $1 \mathrm{GeV}$ within $\Delta R<0.2$ of the seed track. Once the full set of tracks for a $\tau$ candidate is established, an associated calorimeter cluster is searched for within $\Delta R<0.2$ of the $p_{\mathrm{T}}$-weighted track barycenter. The existence of an associated cluster is not required. Calorimeter-based candidates are seeded by jets reconstructed from calibrated topological clusters [25] with $\Delta R<0.4$ and $E_{\mathrm{T}}>10 \mathrm{GeV}$. Once a seed jet is established the algorithm searches for associated ID tracks having $p_{\mathrm{T}}>1 \mathrm{GeV}$, within a cone of radius 


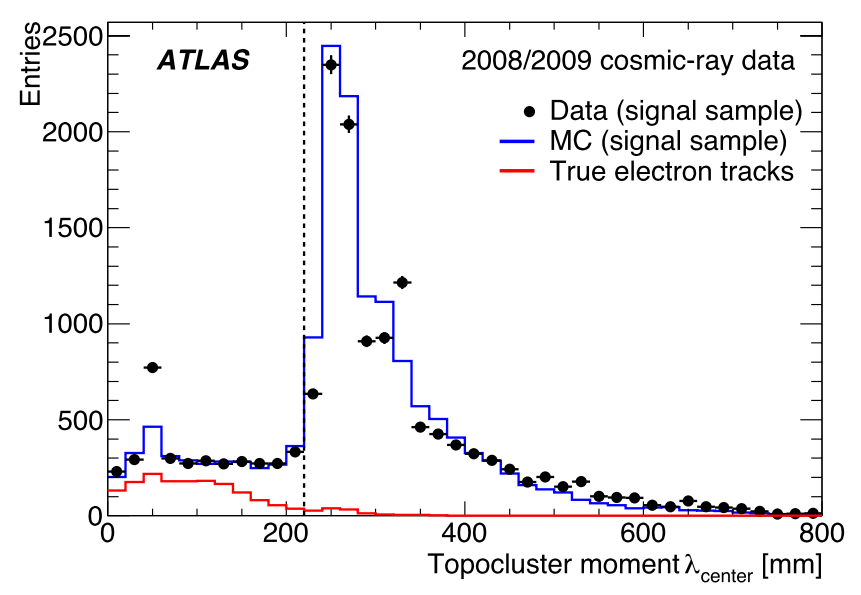

Fig. 12 Data and Monte Carlo distributions for the topological cluster moment $\lambda_{\text {center}}$, for signal and background events from the low- $p_{\mathrm{T}}$ electron selection. The left-hand plot shows the distribution obtained with the signal selection applied to the cosmic-ray data and Monte Carlo, along with the expected distribution for true electrons from the Monte Carlo. The cut at $220 \mathrm{~mm}$ is indicated by the dotted vertical
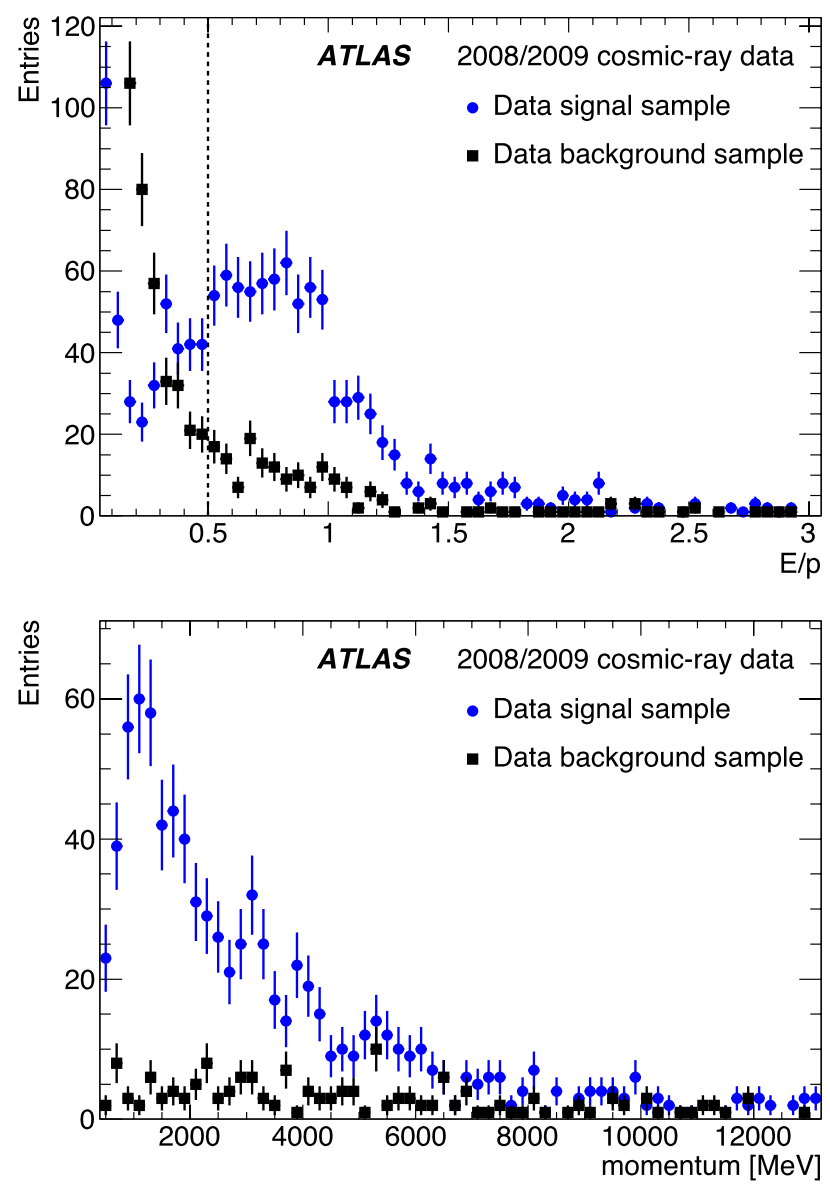

Fig. 13 Results of the selection of low $p_{\mathrm{T}}$ electrons from the cosmicray data samples. The upper plots show the $E / p$ distributions for selected events in data (left) and Monte Carlo (right), for both the signal (ionization) and background (muon-bremsstrahlung) samples, and for

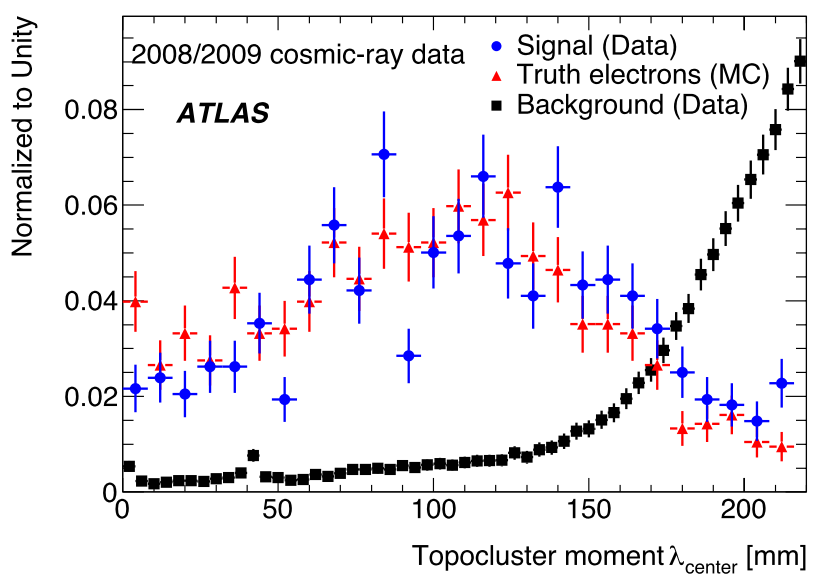

line. For this plot, none of the cluster shape cuts have been applied. The right hand plot shows the selected region after all cuts, for the signal events, the events from the background sample, and for the truth electron distribution from Monte Carlo. The distributions are normalized to unity
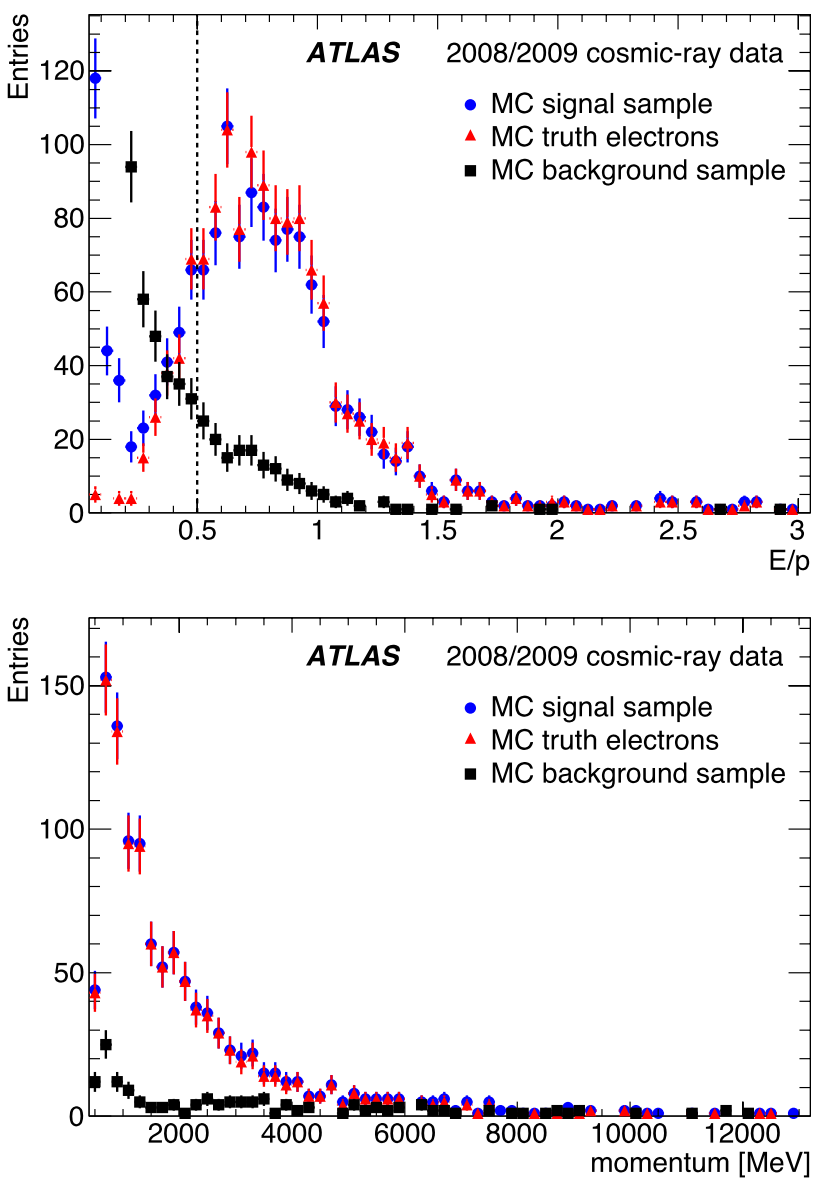

the signal candidates matched to true electrons in the case of the Monte Carlo. The lower plots show the corresponding momentum distributions, for events passing the $E / p$ cut, illustrated by the dashed lines in the upper plots 
$\Delta R<0.3$. The existence of such accompanying tracks is not required.

Since no $\tau$ leptons are expected in the cosmic-ray data sample, the focus of the study described here was simply to exercise the algorithms designed to identify them, and to investigate how well the quantities used for the selection are modeled in the simulation. Since $\tau$ leptons produced in proton-proton collisions originate from the interaction point, these algorithms normally impose tight requirements on the $d_{0}$ and $z_{0}$ parameters of the $\tau$ tracks. However, since application of too tight a selection on these quantities (here with respect to the nominal IP) severely limits the available statistics, in this study acceptance cuts of $\left|d_{0}\right| \leq 40 \mathrm{~mm}$ and $\left|z_{0}\right| \leq 200 \mathrm{~mm}$ were used. These define a region which is well within the sensitive volume of the barrel part of the Pixel Detector, which extends to $r=123 \mathrm{~mm}$ and $z= \pm 400 \mathrm{~mm}$.

The analysis described here uses the cosmic-ray data from the fall 2008 run. The PCM dataset was used as the starting point for each study. Additional requirements were placed on the presence of pixel hits, differently for the two seeding methods. The track-based selection required that the seed track have at least one pixel hit. For studies of the calorimeter-seeded algorithm, while there was no explicit requirement on the association of a track to the seed jet, there was a requirement that there be at least one ID track in the event with at least one pixel hit. This track would normally be from the muon responsible for the calorimeter cluster around which the seed jet is formed. However, in cosmic-ray events these tracks are often not associated with the cluster. The pixel hit requirement is thus intended to ensure that the shower shapes (which are used by the identification algorithm) are approximately as expected for particles originating from the IP.

The $\tau$-identification algorithm is designed to reconstruct $\tau$ leptons over a wide spectrum of energies. However, the relative performance of the two seeding methods varies as a function of energy with the track-seeding having better performance at lower energies while for higher energies, the calorimeter-seeding is superior. Because of this, the type of cosmic-ray event producing fake $\tau$ candidates differs for the two seed types. Most fake track-seeded candidates come from minimum-ionizing muons with low momentum, which produce an ID track that fakes a one-prong candidate. The dominant source of calorimeter-seeded fakes is cosmic-ray muons that undergo hard bremsstrahlung in the calorimeter. When considering real $\tau$ leptons reconstructed from collision data, ideally one would like to have candidates seeded simultaneously by the track and cluster-based algorithms. In cosmic-ray data, however, since the origin of fake $\tau$ leptons differs for each algorithm, very few candidates fulfil the criteria for both. For this reason, track-seeded and calorimeterseeded $\tau$ candidates have been examined separately.
Results are presented here to illustrate the agreement between data and cosmic-ray Monte Carlo for the properties of the two types of $\tau$ candidates, in particular for those quantities used in the identification algorithms. In what follows it should be understood that " $\tau$ candidate" refers to a fake candidate that passes the selection described above, in which nominal selection criteria have been loosened to ensure sufficient statistics to allow for a meaningful comparison of the data and the cosmic-ray Monte Carlo simulation. For track-seeded candidates, Fig. 14 shows the $E_{\mathrm{T}}$ distribution and the distribution of the invariant mass of the charged and neutral constituents. These quantities are both obtained via an energy-flow algorithm [24] in which the energies of calorimeter clusters associated with tracks have been replaced by the corresponding track momenta as measured in the ID. Good agreement is seen between the cosmic-ray data and the simulation.

Figure 15 shows data versus Monte Carlo comparisons for some of the quantities used for the identification of calorimeter-seeded $\tau$ candidates. The upper left plot shows the $E_{\mathrm{T}}$ distribution. The upper right plot shows the isolation fraction, which is a measure of the collimation of the $\tau$-jet, defined as a ratio in which the denominator is the energy deposited within a cone (around the $\tau$ direction) of $\Delta R<0.4$ and the numerator is the energy deposited in the region $0.1<\Delta R<0.2$. The lower left plot shows the centrality fraction, defined as the ratio of the energy within a cone of $\Delta R<0.1$ to that within a cone of $\Delta R<0.4$. The lower right plot shows the distribution of the hadronic radius, which is the energy-weighted width of the cluster, calculated from the energy and positions of the constituent calorimeter cells, relative to the cluster center. All distributions show good agreement between the data and the simulation. In the upper right plot of Fig. 15 there are entries at negative values that are attributable to the noise. This is also the cause of the entries at values greater than 1 in the plot of the centrality fraction. The agreement between data and simulation in these regions illustrates that the modeling of the electronic noise in the simulation is reasonable.

\section{Jet and missing transverse energy studies using cosmic-ray events}

Numerous theories of physics beyond the Standard Model predict the existence of massive weakly interacting particles that escape detection and thus leave a large energy imbalance in the detector. For this reason, detailed understanding of the detector performance for missing transverse energy $\left(E_{\mathrm{T}}^{\mathrm{miss}}\right)$ is extremely important. The most important input to the calculation of the $E_{\mathrm{T}}^{\text {miss }}$ comes from the calorimeter, which provides coverage in the region of $|\eta|<4$.9. Cosmicray energy deposits in the calorimeter typically lead to an 


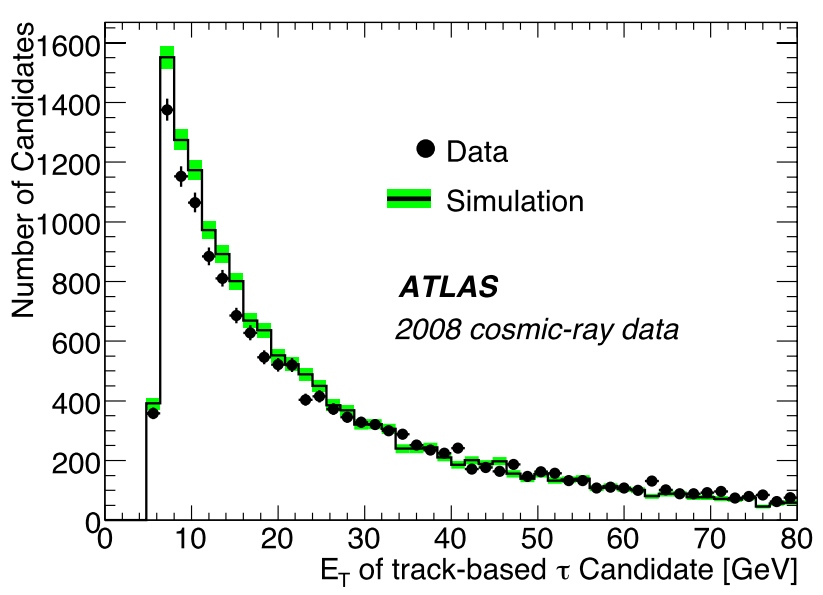

Fig. 14 Data and Monte Carlo distributions for track-seeded $\tau$ candidates reconstructed from cosmic-ray data. The left-hand plot shows the $E_{\mathrm{T}}$ distribution, while the right-hand plot shows the invariant mass
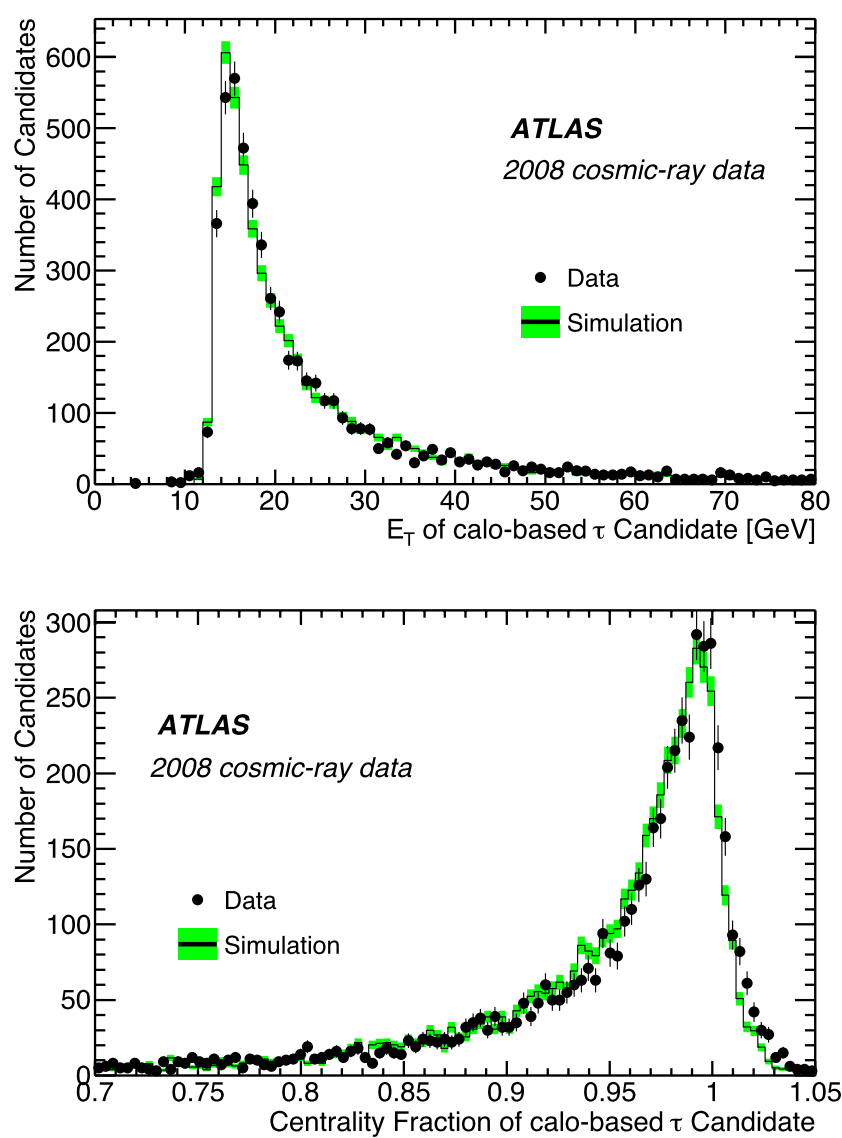

Fig. 15 Data and Monte Carlo distributions for calorimeter-seeded $\tau$ candidates reconstructed from cosmic-ray data. The upper left plot shows the $E_{\mathrm{T}}$ distribution of all candidates, while the other three plots show distributions of quantities used by the $\tau$-identification algorithm. The upper right plot shows the isolation fraction, defined as the ratio in which the denominator is the energy deposited within a cone (around the $\tau$ direction) of $\Delta R<0.4$ and the numerator is the energy deposited

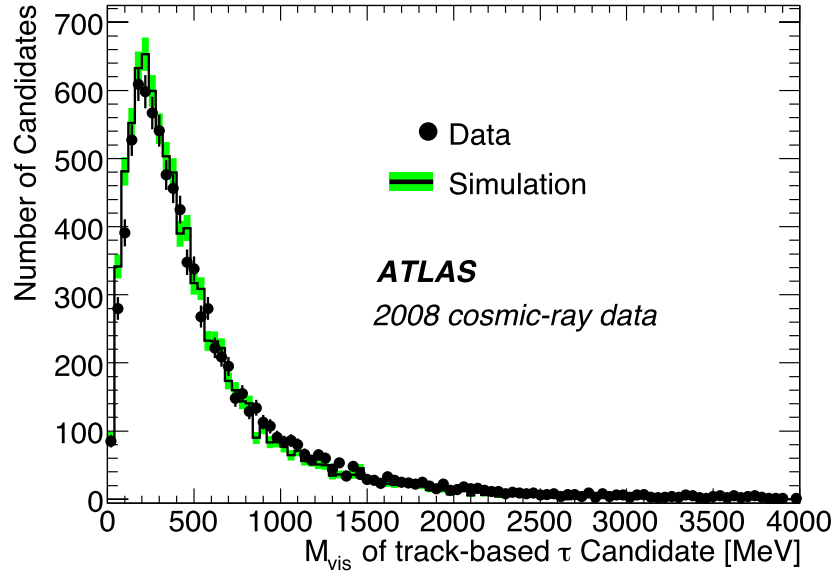

of the charged and neutral constituents. In each case, an energy-flow algorithm was employed, as described in the text
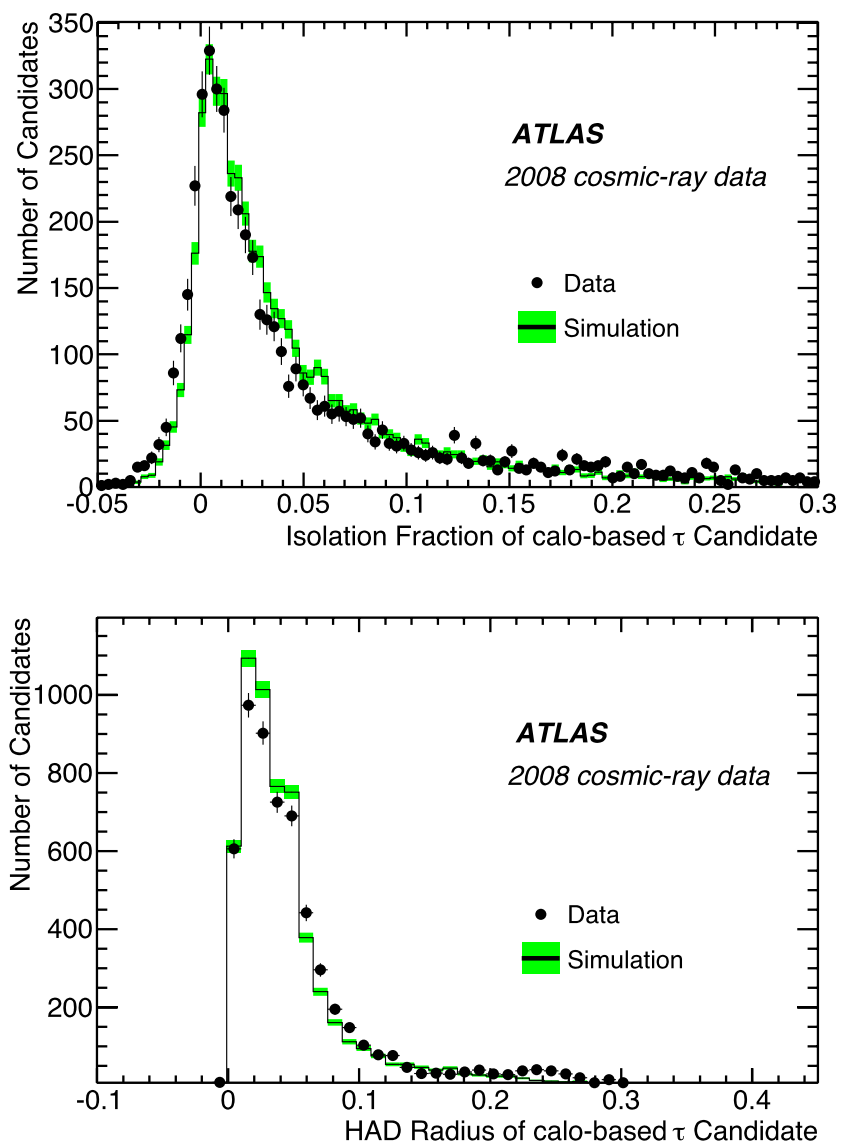

in the region $0.1<\Delta R<0.2$. The lower left plot shows the centrality fraction, defined as the ratio of the energy within a cone of $\Delta R<0.1$ to that within a cone of $\Delta R<0.4$. The lower right plot shows the distribution of the hadronic radius, the energy-weighted width of the cluster, calculated from the energy and positions of the constituent calorimeter cells, relative to the cluster center 
imbalance in the transverse energy in the event. This effect can be large in the case of high-energy cosmic rays that lose a large amount of energy via bremsstrahlung. The energy deposits from cosmic-ray muons (or cosmic-induced airshower events) can be reconstructed as jets, creating backgrounds to jet selections in many analyses. The properties of jets and $E_{\mathrm{T}}^{\text {miss }}$ reconstructed from cosmic-ray data are presented below, along with a discussion of techniques that have been developed to suppress such contributions in the analysis of collision data.

\subsection{Missing transverse energy in randomly-triggered events}

As is the case when running with proton-proton collisions, during cosmic-ray data-taking randomly triggered events are also recorded. The large sample of such events collected during the global cosmic-ray running allows investigations of the detector performance for the measurement of missing transverse energy. No energy imbalance is expected in these events. However, global quantities such as $E_{\mathrm{T}}^{\text {miss }}$ and $\sum E_{\mathrm{T}}$ (defined below) result from the sum of energy deposits in $\sim 200 \mathrm{k}$ calorimeter channels, each with its own electronic noise. A proper determination of these quantities relies on a good understanding of the cell-level noise in all calorimeter channels, and, in particular, a proper treatment of a few very noisy cells and cells having non-nominal high-voltage. There are currently two standard methods for reconstructing missing transverse energy in ATLAS. The first is a celllevel method that takes as input all calorimeter cells with $|E|>2 \sigma_{\text {noise }}$. The second method takes as input calibrated topological clusters built with $t_{\text {seed }}=4, t_{\text {neighbor }}=2$ and $t_{\text {cell }}=0$. The reconstructed quantities are:

$$
\begin{aligned}
& E_{\mathrm{x}}^{\mathrm{miss}}=-\sum E \sin \theta \cos \phi, \\
& E_{\mathrm{y}}^{\mathrm{miss}}=-\sum E \sin \theta \sin \phi, \\
& E_{\mathrm{T}}^{\mathrm{miss}}=\left(\left(E_{\mathrm{x}}^{\mathrm{miss}}\right)^{2}+\left(E_{\mathrm{y}}^{\mathrm{miss}}\right)^{2}\right)^{1 / 2}, \\
& \sum E_{\mathrm{T}}=\sum E \sin \theta,
\end{aligned}
$$

where in each case the sum is over all cells included in the cluster.

Figure 16 shows the results of both calculations applied to the random triggers recorded during a 2008 cosmic-ray run, illustrating the superior noise suppression of the method using the topological clustering. Tails in the distribution (beyond $8 \mathrm{GeV}$ for topological-cluster-based, and $16 \mathrm{GeV}$ for cell-based definition), contributing less than $0.1 \%$ of events, are due to coherent noise in a specific region of the LAr presampler. This problem was repaired prior to first collisions, as can be seen in [26]. The time stability of the $E_{\mathrm{T}}^{\text {miss }}$ calculation was also investigated and found to be very good.

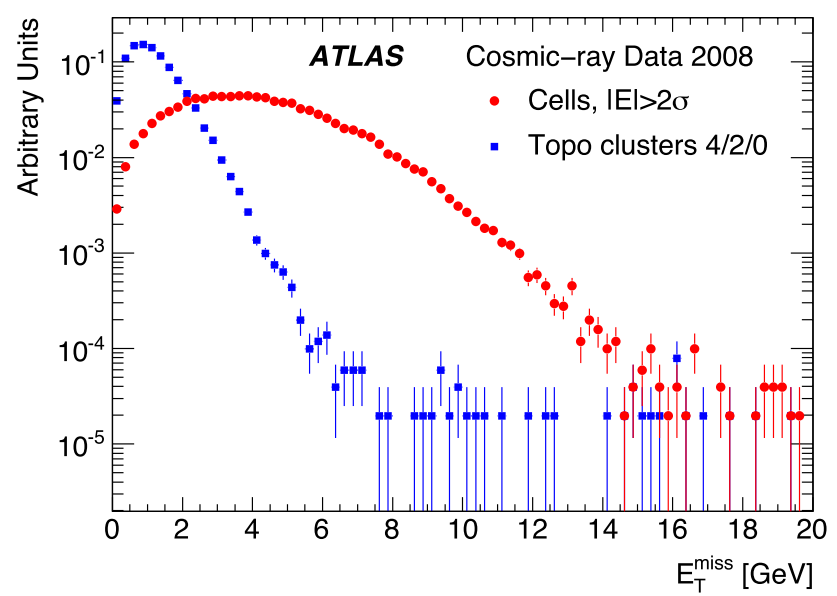

Fig. 16 Distribution of $E_{\mathrm{T}}^{\text {miss }}$ from analysis of random triggers recorded during the 2008 global cosmic-ray running, for the two methods described in the text

For the topological-cluster-based method, which provides the best resolution, the mean and width of the distributions of the $x$ and $y$ components of $E_{\mathrm{T}}^{\text {miss }}$ were stable to within about $100 \mathrm{MeV}$ over the 45 days of data-taking.

\subsection{Jets and missing transverse energy in cosmic-ray events}

The reconstruction of jets and $E_{\mathrm{T}}^{\text {miss }}$ in cosmic-ray events has been studied using the L1Calo-triggered data taken in the 2008 and 2009 cosmic-ray runs. For jet reconstruction an anti- $k_{\mathrm{t}}$ algorithm [27] is employed, with calibrated topological clusters as input. Figure 17 shows the distributions of missing transverse energy and summed transverse energy from cosmic-ray events having a reconstructed jet with $p_{\mathrm{T}}>20 \mathrm{GeV}$. The 2008 and 2009 data samples are shown separately to demonstrate the consistency of the two samples. The distributions from the 2008 data and the cosmicray Monte Carlo are normalized to that of the 2009 data in the region of $100<E_{\mathrm{T}}^{\text {miss }}<300 \mathrm{GeV}$. This is in order to avoid any threshold effects, since the trigger was not simulated in the cosmic-ray Monte Carlo sample. In each case there is agreement with the shape expected from the Monte Carlo, which requires an understanding of the electronic noise in each calorimeter channel. The upper left plot in Fig. 18 shows the corresponding $p_{\mathrm{T}}$ distribution of the jets reconstructed in this sample.

Suppression of these fake jet candidates can be performed using a selection based on three quantities:

$$
\begin{aligned}
& R_{\mathrm{J}}=\sum_{i=1}^{N} \sqrt{\left(\eta_{i}-\eta_{\mathrm{jet}}\right)^{2}+\left(\phi_{i}-\phi_{\mathrm{jet}}\right)^{2}} \cdot E_{i} / \sum_{i=1}^{N} E_{i}, \\
& R_{\mathrm{LC}}=\left(\sum_{i=1}^{2} E_{i}^{\mathrm{Had}}+\sum_{i=1}^{32} E_{i}^{\mathrm{EM}}\right) / \sum_{i=1}^{N} E_{i}, \\
& f_{\mathrm{EM}}=E_{\mathrm{EM}} /\left(E_{\mathrm{EM}}+E_{\mathrm{Had}}\right) .
\end{aligned}
$$




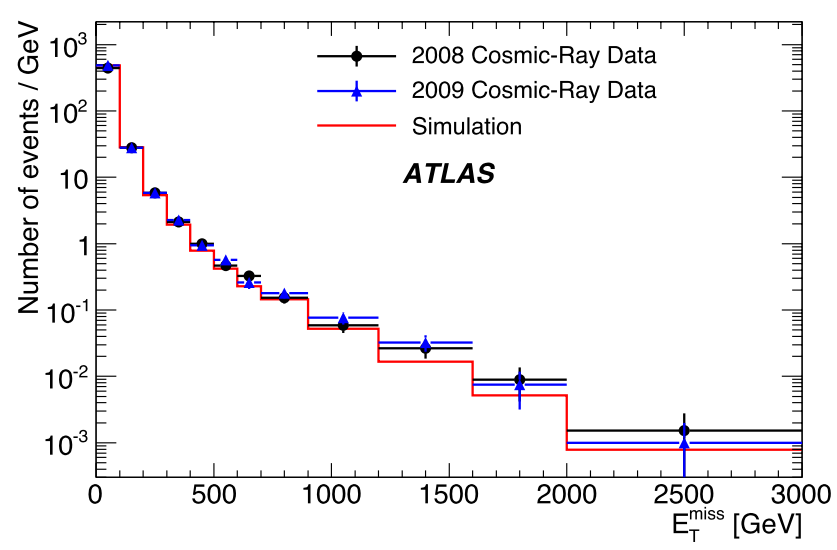

Fig. 17 Distributions of $E_{\mathrm{T}}^{\text {miss }}(l e f t)$ and $\sum E_{\mathrm{T}}$ (right) from analysis of the 2008 and 2009 L1Calo-triggered cosmic-ray data and from the cosmic-ray Monte Carlo sample. The 2008 and Monte Carlo dis-
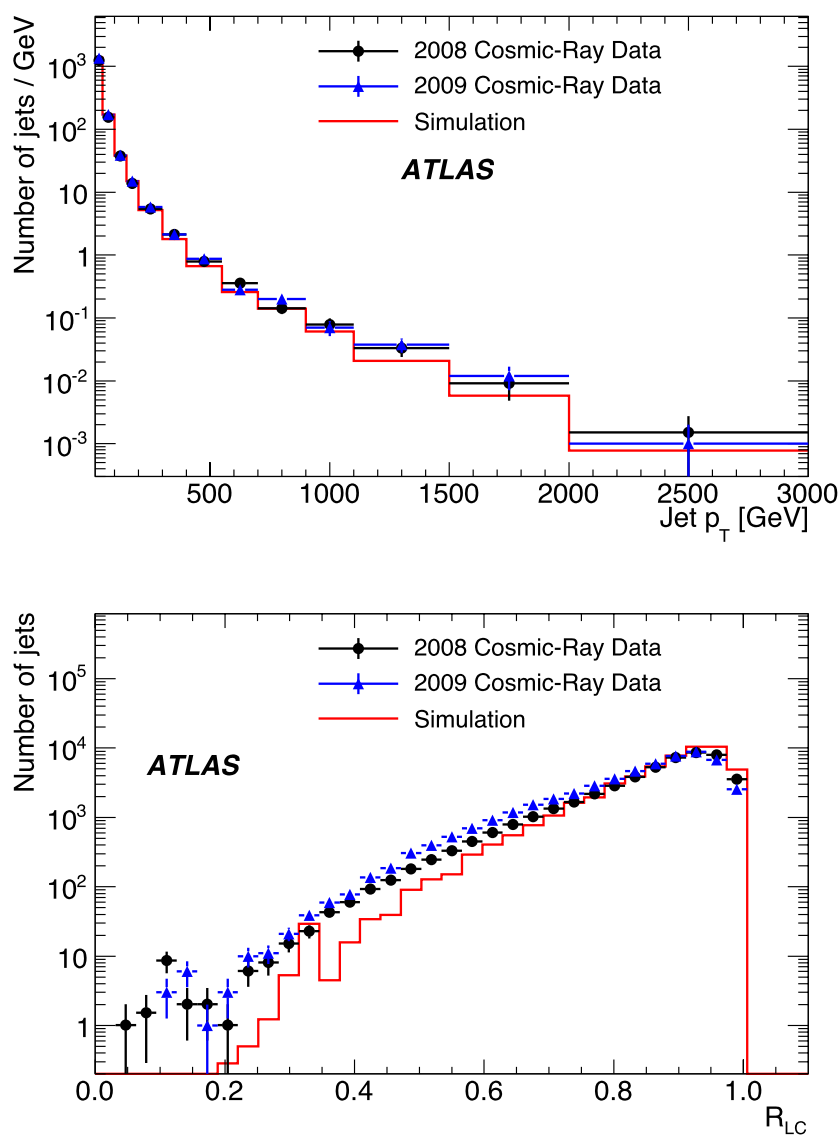

Fig. 18 Properties of fake jets reconstructed from the 2008 and 2009 L1Calo-triggered cosmic-ray data samples: The upper left plot shows the jet $p_{\mathrm{T}}$ in the acceptance region above $20 \mathrm{GeV}$, while the other three

Here $R_{\mathrm{J}}$ represents the energy-weighted lateral extent of the jet, in $\eta \times \phi$ space. $R_{\mathrm{LC}}$ represents the fraction of the jet energy contained in the "leading cells", defined as the two most energetic cells in the hadronic calorimeter and the 32

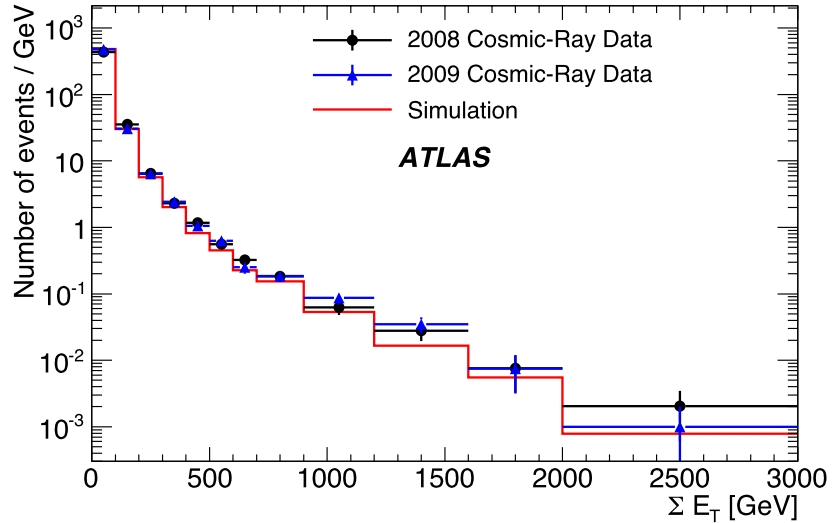

tributions are normalized to the 2009 data distribution in the region $100<E_{\mathrm{T}}^{\mathrm{miss}}<300 \mathrm{GeV}$
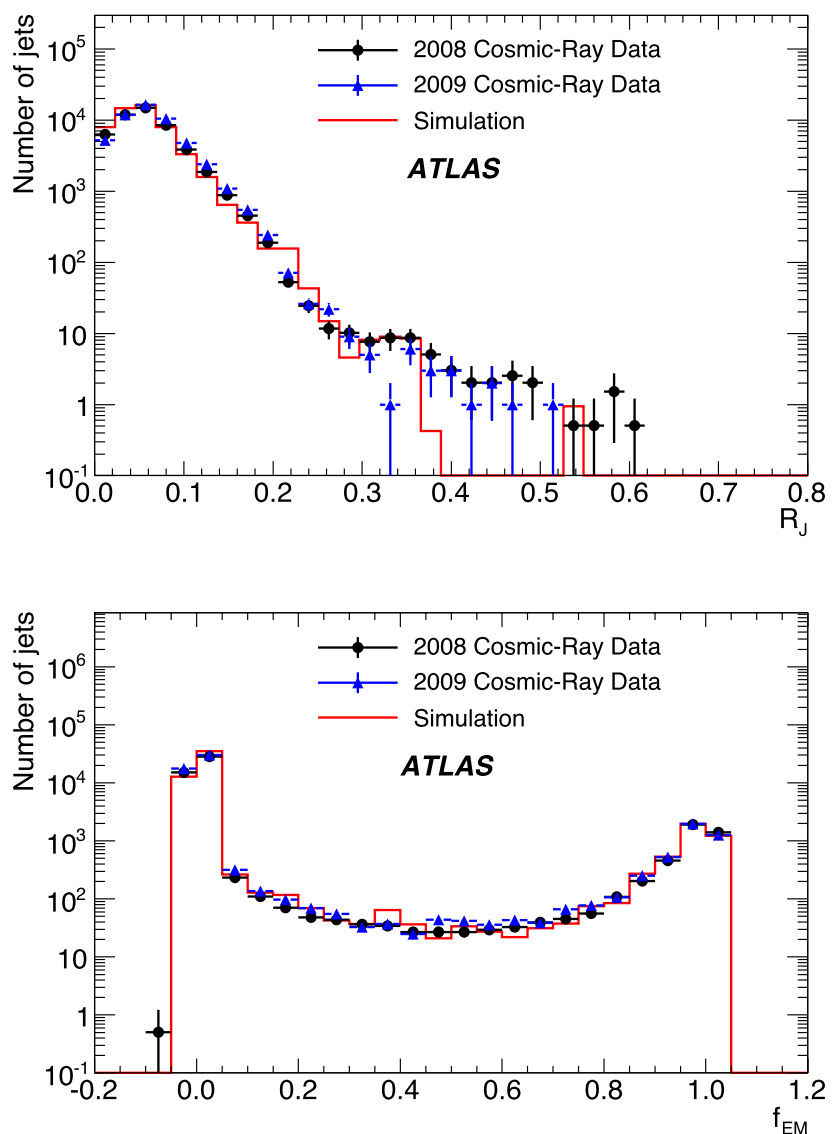

plots show distributions in quantities used to suppress these contributions in collision data, as described in the text. The normalizations are the same as used in Fig. 17

most energetic cells in the EM calorimeter, where the sum in the denominator is over all $N$ calorimeter cells associated with the jet candidate. Finally, $f_{\mathrm{EM}}$ represents the electromagnetic fraction of the jet, defined as the fraction of the jet 
energy that is deposited in the EM calorimeter. The distributions of these three quantities for the selected jets are also shown in Fig. 18. Again there is good agreement between the 2008 and 2009 cosmic-ray data as well as reasonable agreement with the cosmic-ray Monte Carlo. The normal-

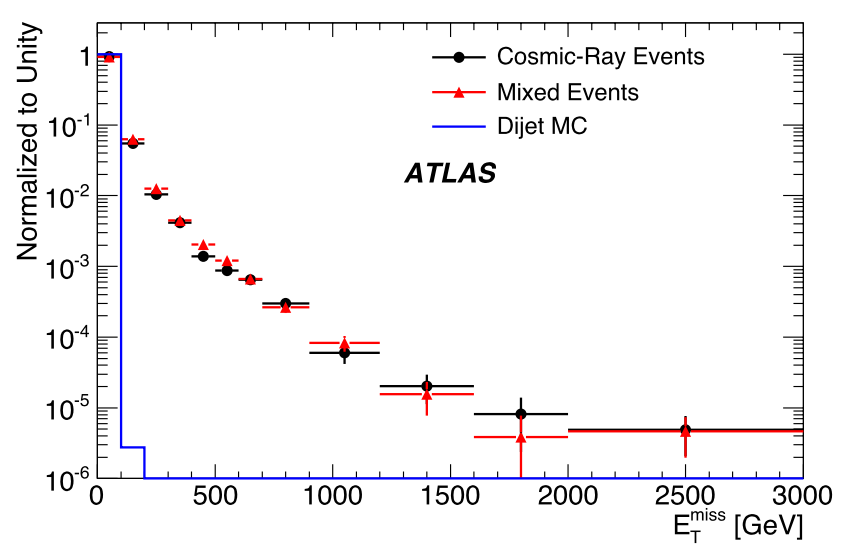

Fig. 19 The same distributions as presented in Fig. 17, obtained from the cosmic-ray data and from the mixed data sample described in the text. The plots are normalized to allow comparison of the shapes of the
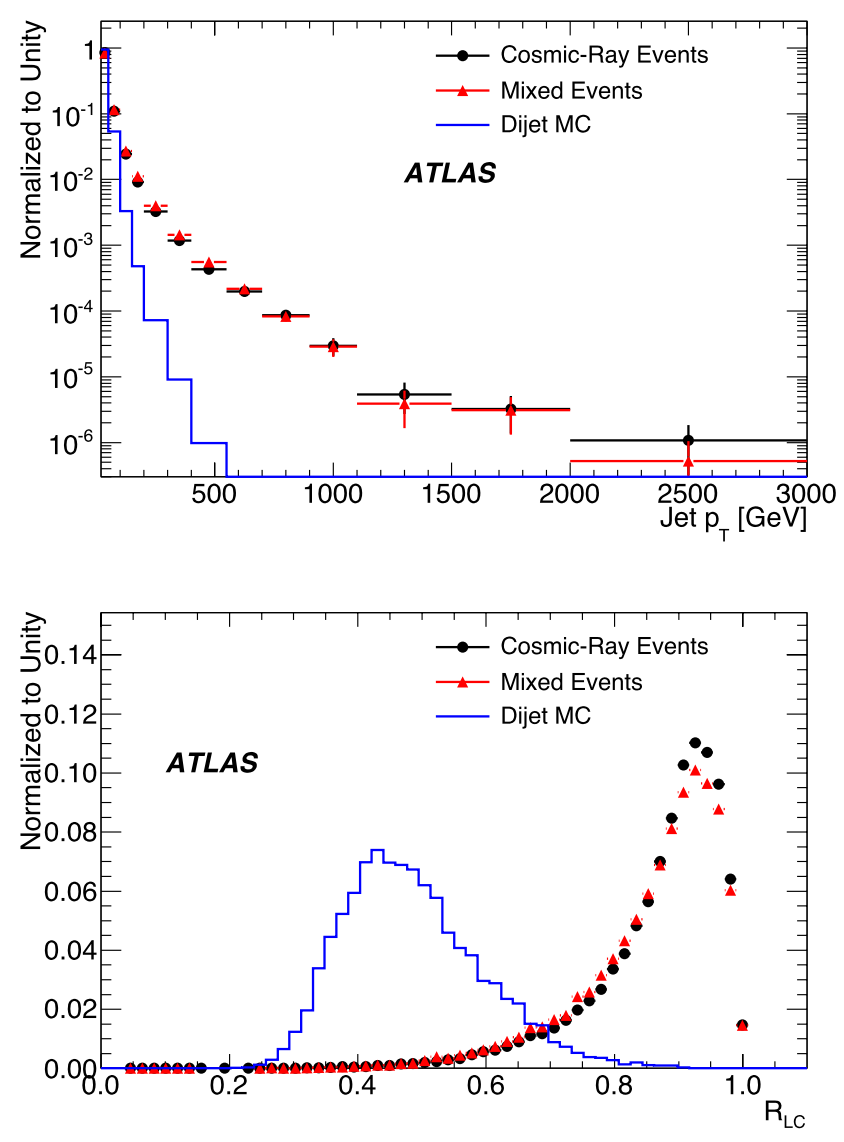

ization of the distributions in Fig. 18 is the same as used in Fig. 17.

When operating ATLAS for proton-proton collisions, contributions from cosmic-ray events can either trigger readout of the detector, or overlap with a triggered collision

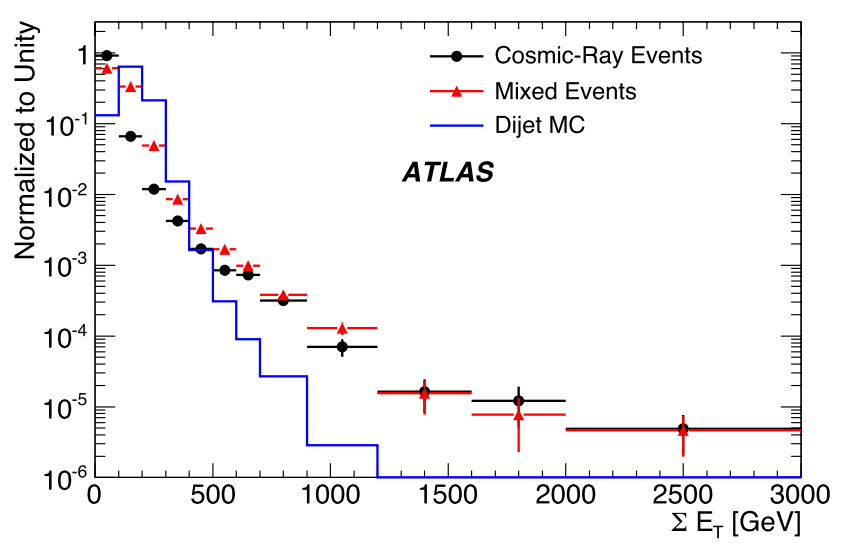

two distributions. Also shown are the corresponding distributions from dijet Monte Carlo events
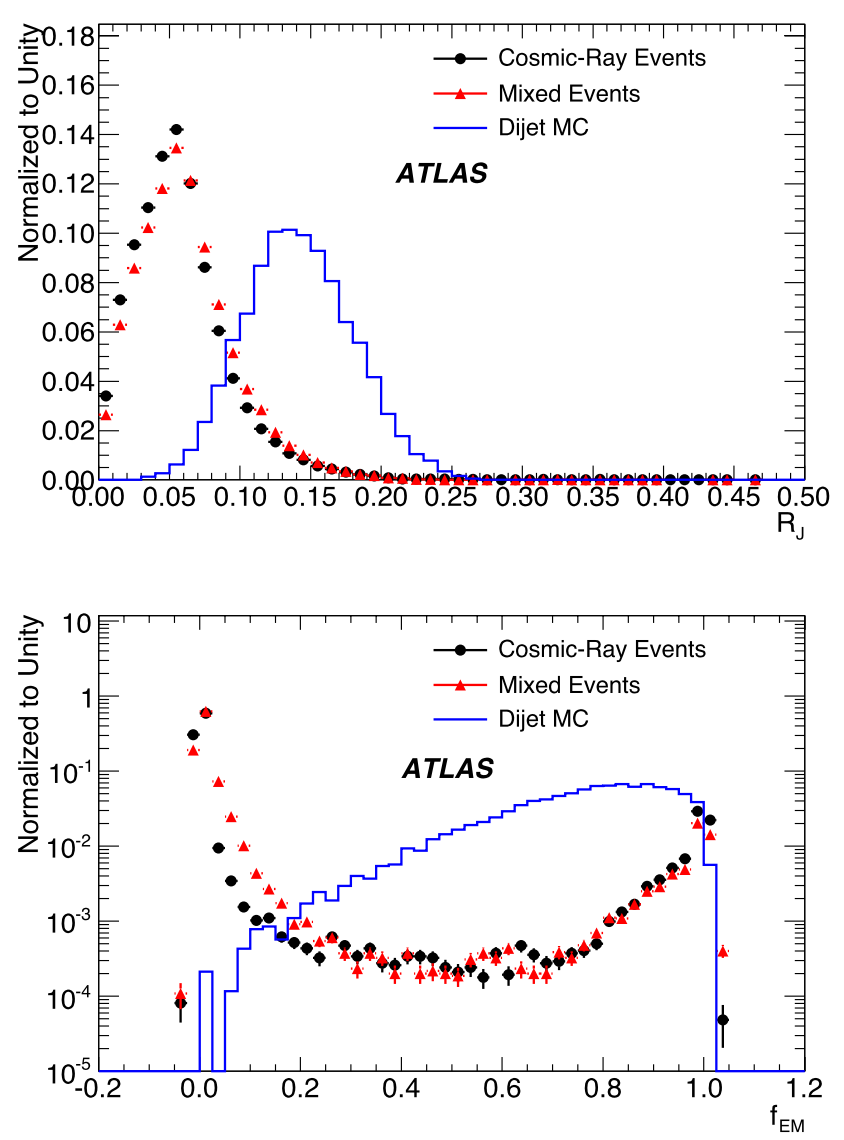

Fig. 20 The same distributions as presented in Fig. 18, obtained from the cosmic-ray data and from the mixed data sample. The plots are normalized to allow comparison of the shapes. Also shown are the corresponding distributions from dijet Monte Carlo events 
event. Since cosmic-ray energy deposits in the latter category may be more difficult to identify, this scenario has been studied using a special data sample in which cosmic-ray events from the 2008 data were overlaid with Monte Carlo minimum-bias events. The overlay is done only with single minimum-bias events, so cannot account for events with pileup. However, in terms of faking a missing $E_{\mathrm{T}}$ signal, one might expect that the relative contribution, of a single cosmic-ray event, to a collision event would be highest in the case of overlap with a single collision. The effect of this additional energy on the $E_{\mathrm{T}}^{\text {miss }}$ and $\sum E_{\mathrm{T}}$ distributions is illustrated in Fig. 19 which compares the distributions obtained from the mixed sample to those obtained from cosmic-ray data alone. The corresponding distributions obtained from a dijet Monte Carlo sample are also shown. In each case the distributions are obtained from all events having a jet with $p_{\mathrm{T}}>20 \mathrm{GeV}$ and $|\eta|<2.5$. They are shown normalized to unity to allow better comparison of the shapes. The effect of the additional energy from the minimum-bias event is apparent in the $\sum E_{\mathrm{T}}$ distribution, at low values.

The mixed data sample was used to investigate the robustness of the jet-discrimination variables in the case where a cosmic-ray event is overlaid with a minimum-bias event. The distributions shown in Fig. 20 are for the same quantities shown in Fig. 18, now normalized to unity. Each plot shows the distribution obtained from cosmic-ray data, from the mixed sample and from a sample of Monte Carlo dijet events. For the three variables introduced earlier, comparison of the distributions obtained from the two samples shows these variables to be robust against the presence of the additional energy due to the minimum-bias event. This was not the case for other discriminating variables (e.g., the number of clusters or tracks included in jets) that were also investigated. Rejection of fake jets from cosmic-ray events can be performed using a log-likelihood ratio (LLR) based on input probability distribution functions (pdfs) from the mixed sample and the Monte Carlo dijet sample. As investigations of the three discriminating variables showed a high degree of correlation between $R_{\mathrm{J}}$ and $R_{\mathrm{LC}}$, a 2-dimensional pdf for these two variables was employed along with a onedimensional pdf for $f_{\mathrm{EM}}$.

Figure 21 illustrates the effects of different applications of "cleaning cuts" based on these pdfs. The upper plot shows the cumulative effect of successive applications of the two LLR cuts on the $p_{\mathrm{T}}$ distribution from the dijet sample and on the fake jet $p_{\mathrm{T}}$ distribution from cosmic-ray events. For the chosen cuts, the effect of each cut on the dijet sample is at the $2 \%$ level in each of the $p_{\mathrm{T}}$ bins. The middle plot compares the effect of the same cuts on the mixed and cosmic-ray data samples. The lower plot shows the rejection factor for events with jets produced by cosmic-ray interactions plotted against the efficiency for the selection of Monte Carlo dijet events, in the acceptance region previously defined, for three different scenarios:
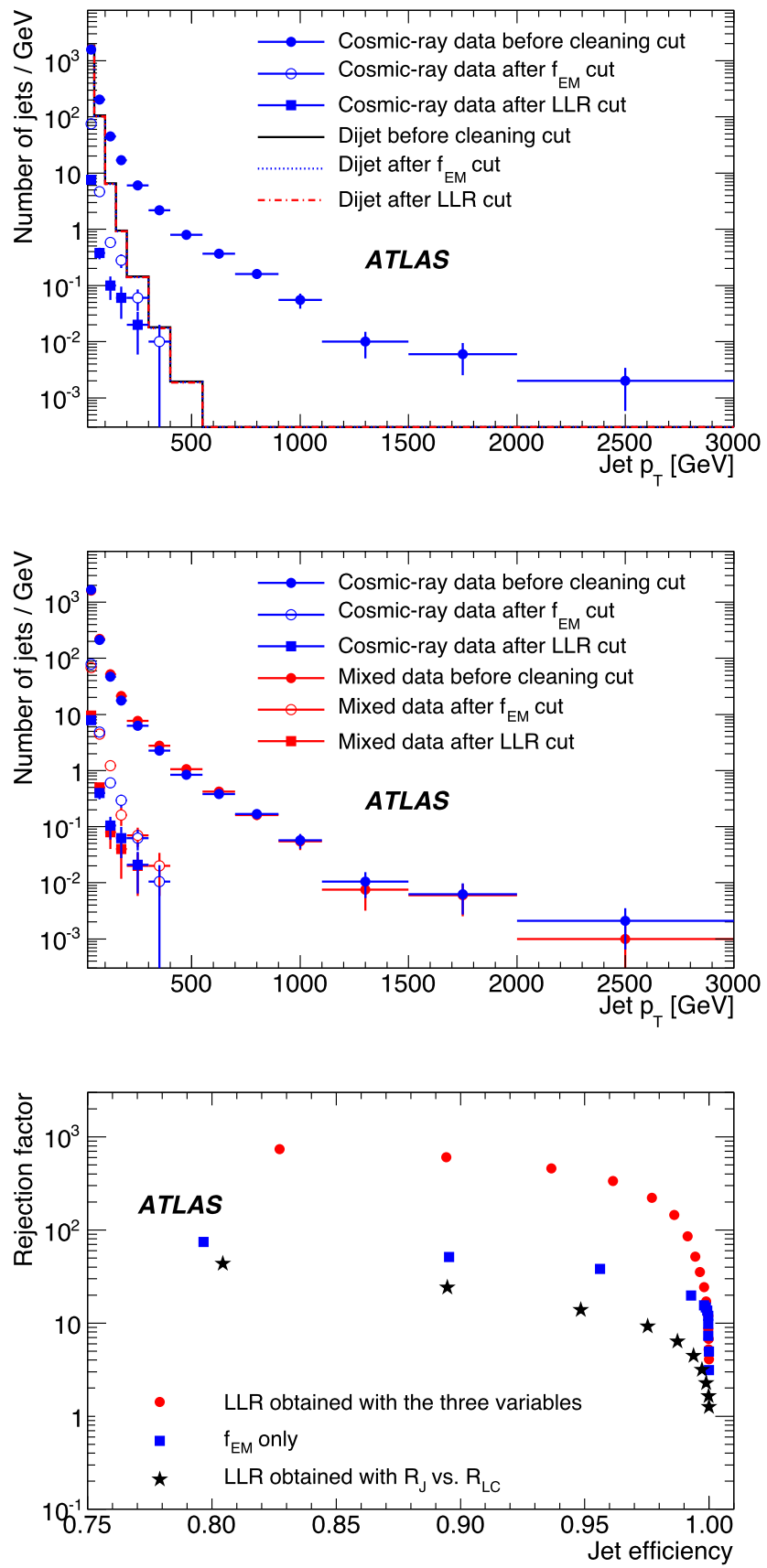

Fig. 21 Performance of the cleaning cuts for the suppression of fake jets from cosmic-ray events. The upper plot shows the effect of the cleaning cuts on the $p_{\mathrm{T}}$ distribution of accepted jets, for different cleaning cuts, for the cosmic-ray and dijet Monte Carlo samples. The middle plot shows similar distributions, this time comparing the mixed events to those obtained using only cosmic-ray data. The lower plot shows the achievable cosmic-ray rejection vs. the efficiency for dijet events

- application of an LLR cut based on $f_{\mathrm{EM}}$ only

- application of a LLR cut based only on $R_{\mathrm{J}}$ and $R_{\mathrm{LC}}$

- application of the full, three-variable LLR.

The rejection factor is obtained from an analysis of the mixed sample while the efficiency is derived from applica- 
tion of the selection to the dijet Monte Carlo sample. An overall rejection factor of about 400 can be obtained with 95\% efficiency for jets from the dijet Monte Carlo sample. For cosmic-ray events without overlaid minimum-bias energy, the situation is somewhat better, with a rejection factor (again for 95\% efficiency for jets in dijet events) of around 550 .

\section{Summary}

Cosmic-ray interactions provide a source of physics signals in the ATLAS detector that have allowed for investigations of the detector alignment, calibration and performance prior to the arrival of first LHC beams. Such events have been used to exercise the detector readout and associated data-handling infrastructure, and the accumulated datasets have been exploited for both standalone and combined performance studies of the detector subsystems. Cosmic-ray data will continue to be relevant to the commissioning of the muon spectrometer until a sufficient number of high$p_{\mathrm{T}}$ muons have been accumulated from proton-proton collisions. In this paper, results relevant to lepton identification and reconstruction as well as the measurement of missing transverse energy were presented, along with studies related to the rejection of background from cosmic-ray events in collision data. These results, along with those presented in the publications describing results from subsystem-specific cosmic-ray commissioning, demonstrate that ATLAS was prepared for the first collisions from the LHC. Measured distributions obtained from analysis of the cosmic-ray data agree well with the predictions of the detector simulation and a dedicated cosmic-muon event generator, demonstrating that the modeling of the detector response was also in good shape prior to first collisions.

Acknowledgements We are greatly indebted to all CERN's departments and to the LHC project for their immense efforts not only in building the LHC, but also for their direct contributions to the construction and installation of the ATLAS detector and its infrastructure. We acknowledge equally warmly all our technical colleagues in the collaborating Institutions without whom the ATLAS detector could not have been built. Furthermore we are grateful to all the funding agencies which supported generously the construction and the commissioning of the ATLAS detector and also provided the computing infrastructure.

The ATLAS detector design and construction has taken about fifteen years, and our thoughts are with all our colleagues who sadly could not see its final realisation.

We acknowledge the support of ANPCyT, Argentina; YerPhI, Armenia; ARC, Australia; BMWF, Austria; ANAS, Azerbaijan; SSTC, Belarus; CNPq and FAPESP, Brazil; NSERC, NRC and CFI, Canada; CERN; CONICYT, Chile; CAS, MOST and NSFC, China; COLCIENCIAS, Colombia; MEYS (MSMT), MPO and CCRC, Czech Republic; DNRF, DNSRC and Lundbeck Foundation, Denmark; ARTEMIS, European Union; IN2P3-CNRS, CEA-DSM/IRFU, France; GNAS, Georgia; BMBF, DFG, HGF, MPG and AvH Foundation, Germany; GSRT, Greece; ISF, MINERVA, GIF, DIP and Benoziyo Center, Israel; INFN, Italy; MEXT and JSPS, Japan; CNRST, Morocco; FOM and NWO,
Netherlands; RCN, Norway; MNiSW, Poland; GRICES and FCT, Portugal; MERYS (MECTS), Romania; MES of Russia and ROSATOM, Russian Federation; JINR; MSTD, Serbia; MSSR, Slovakia; ARRS and MVZT, Slovenia; DST/NRF, South Africa; MICINN, Spain; SRC and Wallenberg Foundation, Sweden; SER, SNSF and Cantons of Bern and Geneva, Switzerland; NSC, Taiwan; TAEK, Turkey; STFC, the Royal Society and Leverhulme Trust, United Kingdom; DOE and NSF, United States of America.

Open Access This article is distributed under the terms of the Creative Commons Attribution Noncommercial License which permits any noncommercial use, distribution, and reproduction in any medium, provided the original author(s) and source are credited.

\section{References}

1. G. Aad et al., The ATLAS experiment at the CERN large hadron collider. J. Instrum. 3, S08003 (2008)

2. L. Evans, P. Bryant, LHC machine. J. Instrum. 3, S08001 (2008)

3. S. Agostinelli et al., GEANT4: A simulation toolkit. Nucl. Instrum. Methods A 506, 250-303 (2003)

4. J. Allison et al., Geant 4 developments and applications. IEEE Trans. Nucl. Sci. 53, 270 (2006)

5. G. Aad et al., The ATLAS simulation infrastructure. Eur. Phys. J. C 70, 823 (2010)

6. G. Aad et al., The ATLAS inner detector commissioning and calibration. Eur. Phys. J. C 70, 787 (2010)

7. G. Aad et al., Readiness of the ATLAS liquid argon calorimeter for LHC collision. Eur. Phys. J. C 70, 723 (2010)

8. G. Aad et al., Readiness of the ATLAS tile calorimeter for LHC collisions. Eur. Phys. J. C 70, 1193 (2010)

9. G. Aad et al., Commissioning of the ATLAS muon spectrometer with cosmic ray muons. Eur. Phys. J. C 70, 875 (2010)

10. S. Fratina et al., The ATLAS transition radiation detector (TRT) fast-OR trigger, ATLAS-INDET-PUB-2009-002, CERN, Geneva, 2009

11. G. Aad et al., Drift time measurement in the ATLAS liquid argon electromagnetic calorimeter using cosmic muons. Eur. Phys. J. C 70, 755 (2010)

12. A. Dar, Atmospheric neutrinos, astrophysical neutrinos and proton decay experiments. Phys. Rev. Lett. 51, 227 (1983)

13. C. Amsler et al., Review of particle physics. Phys. Lett. B 667, 1 (2008)

14. M. Boonekamp et al., Cosmic ray, beam halo and beam gas rate studies for ATLAS commissioning, ATL-GEN-2004-001, CERN, Geneva, 2004

15. H. Okawa, Commissioning of the ATLAS calorimeters at the large hadron collider and prospects towards new physics search, Ph.D. thesis, University of Tokyo, Japan, 2010

16. D. Adams et al., Track reconstruction in the ATLAS muon spectrometer with MOORE, ATL-SOFT-2003-007, CERN, Geneva, Oct 2003

17. T. Cornelissen et al., Concepts, design and implementation of the ATLAS new tracking (NEWT), ATL-SOFT-PUB-2007-007, CERN, Geneva, 2007

18. T. Cornelissen et al., The new ATLAS track reconstruction (NEWT). J. Phys. Conf. Ser. 119, 032013 (2008)

19. K. Bachas, S. Hassani, Track based software package for measurement of the energy deposited by muons in the calorimeters of the ATLAS detector. J. Phys. Conf. Ser. 119, 042002 (2008)

20. B. Lenzi, R. Nicolaidou, S. Hassani, TrackInCaloTools: A package for measuring muon energy loss and calorimetric isolation in ATLAS. J. Phys. Conf. Ser. 119, 032049 (2010) 
21. B. Lenzi, Search for the Higgs boson decaying to four leptons in the ATLAS detector at LHC and studies of muon isolation and energy loss, Ph.D. thesis, Université Paris Sud Orsay, France, 2010

22. A. Salzburger, The ATLAS track extrapolation package, ATLSOFT-PUB-2007-005, CERN, Geneva, Jun 2007

23. A. Salzburger, S. Todorova, M. Wolter, The ATLAS tracking geometry description, ATL-SOFT-PUB-2007-004, CERN, Geneva, Jun 2007

24. G. Aad et al., Expected Performance of the ATLAS ExperimentDetector, Trigger and Physics, CERN-OPEN 2008-020, Dec.
2008, ISBN 978-92-9083-321-5, arXiv:0901.0512 [hep-ex] (2009)

25. T. Barillari et al., Local hadronic calibration, ATL-LARG-PUB2009-001-2 CERN, Geneva, 2010

26. G. Aad et al., Performance of the ATLAS detector using first collision data. J. High Energy Phys. 1009, 056 (2010)

27. M. Cacciari, G.P. Salam, G. Soyez, The anti- $k_{t}$ jet clustering algorithm. J. High Energy Phys. 04, 063 (2008)

\section{The ATLAS Collaboration}

G. Aad $^{48}$, B. Abbott ${ }^{111}$, J. Abdallah ${ }^{11}$, A.A. Abdelalim ${ }^{49}$, A. Abdesselam ${ }^{118}$, O. Abdinov ${ }^{10}$, B. Abi ${ }^{112}$, M. Abolins $^{88}$, H. Abramowicz ${ }^{153}$, H. Abreu ${ }^{115}$, B.S. Acharya ${ }^{164 a, 164 b}$, D.L. Adams ${ }^{24}$, T.N. Addy ${ }^{56}$, J. Adelman ${ }^{175}$, S. Adomeit ${ }^{98}$, P. Adragna ${ }^{75}$, T. Adye ${ }^{129}$, S. Aefsky ${ }^{22}$, J.A. Aguilar-Saavedra ${ }^{124 b, a}$, M. Aharrouche ${ }^{81}$, S.P. Ahlen ${ }^{21}$, F. Ahles ${ }^{48}$, A. Ah$\operatorname{mad}^{148}$, M. Ahsan $^{40}$, G. Aielli133a,133b , T. Akdogan ${ }^{18 a}$, T.P.A. Åkesson ${ }^{79}$, G. Akimoto ${ }^{155}$, A.V. Akimov ${ }^{94}$, A. Aktas ${ }^{48}$, M.S. Alam ${ }^{1}$, M.A. Alam ${ }^{76}$, S. Albrand ${ }^{55}$, M. Aleksa ${ }^{29}$, I.N. Aleksandrov ${ }^{65}$, C. Alexa ${ }^{25 a}$, G. Alexander ${ }^{153}$, G. Alexandre $^{49}$, T. Alexopoulos 9 , M. Alhroob ${ }^{20}$, M. Aliev ${ }^{15}$, G. Alimonti ${ }^{89 a}$, J. Alison ${ }^{120}$, M. Aliyev ${ }^{10}$, P.P. Allport ${ }^{73}$, S.E. AllwoodSpiers $^{53}$, J. Almond ${ }^{82}$, A. Aloisio ${ }^{102 a, 102 b}$, R. Alon ${ }^{171}$, A. Alonso ${ }^{79}$, M.G. Alviggi ${ }^{102 a, 102 b}$, K. Amako ${ }^{66}$, C. Amelung ${ }^{22}$, A. Amorim ${ }^{124 a, b}$, G. Amorós ${ }^{167}$, N. Amram ${ }^{153}$, C. Anastopoulos ${ }^{139}$, T. Andeen ${ }^{29}$, C.F. Anders ${ }^{48}$, K.J. Anderson ${ }^{30}$, A. Andreazza $^{89 a, 89 b}$, V. Andrei ${ }^{58 a}$, X.S. Anduaga ${ }^{70}$, A. Angerami ${ }^{34}$, F. Anghinolfi ${ }^{29}$, N. Anjos ${ }^{124 a}$, A. Annovi ${ }^{47}$, A. Antonaki ${ }^{8}$, M. Antonelli ${ }^{47}$, S. Antonelli ${ }^{19 a}$,19b, J. Antos ${ }^{144 b}$, B. Antunovic ${ }^{41}$, F. Anulli ${ }^{132 a}$, S. Aoun ${ }^{83}$, G. Arabidze ${ }^{8}$, I. Aracena ${ }^{143}$, Y. Arai ${ }^{66}$, A.T.H. Arce ${ }^{44}$, J.P. Archambault ${ }^{28}$, S. Arfaoui ${ }^{29, c}$, J.-F. Arguin ${ }^{14}$, T. Argyropoulos ${ }^{9}$, M. Arik ${ }^{18 a}$, A.J. Armbruster $^{87}$, O. Arnaez ${ }^{4}$, C. Arnault ${ }^{115}$, A. Artamonov ${ }^{95}$, D. Arutinov ${ }^{20}$, M. Asai ${ }^{143}$, S. Asai ${ }^{155}$, J. Silva ${ }^{124 a, d}$, R. Asfandiyarov $^{172}$, S. Ask ${ }^{82}$, B. Åsman 146a,146b, D. Asner ${ }^{28}$, L. Asquith ${ }^{77}$, K. Assamagan ${ }^{24}$, A. Astvatsatourov ${ }^{52}$, G. Atoian ${ }^{175}$, B. Auerbach ${ }^{175}$, K. Augsten ${ }^{127}$, M. Aurousseau ${ }^{4}$, N. Austin ${ }^{73}$, G. Avolio ${ }^{163}$, R. Avramidou ${ }^{9}$, C. Ay ${ }^{54}$, G. Azuelos ${ }^{93, e}$, Y. Azuma ${ }^{155}$, M.A. Baak ${ }^{29}$, A.M. Bach ${ }^{14}$, H. Bachacou ${ }^{136}$, K. Bachas ${ }^{29}$, M. Backes ${ }^{49}$, E. Badescu ${ }^{25 a}$, P. Bagnaia ${ }^{132 a, 132 b}$, Y. Bai ${ }^{32 a}$, T. Bain ${ }^{158}$, J.T. Baines ${ }^{129}$, O.K. Baker ${ }^{175}$, M.D. Baker ${ }^{24}$, S. Baker ${ }^{77}$, F. Baltasar Dos Santos Pedrosa ${ }^{29}$, E. Banas ${ }^{38}$, P. Banerjee ${ }^{93}$, Sw. Banerjee ${ }^{169}$, D. Banfi ${ }^{89 a, 89 b}$, A. Bangert ${ }^{137}$, V. Bansal ${ }^{169}$, S.P. Baranov ${ }^{94}$, A. Barashkou ${ }^{65}$, T. Barber ${ }^{27}$, E.L. Barberio ${ }^{86}$, D. Barberis ${ }^{50 a, 50 b}$, M. Barbero ${ }^{20}$, D.Y. Bardin ${ }^{65}$, T. Barillari ${ }^{99}$, M. Barisonzi ${ }^{174}$, T. Barklow ${ }^{143}$, N. Barlow $^{27}$, B.M. Barnett ${ }^{129}$, R.M. Barnett ${ }^{14}$, A. Baroncelli ${ }^{134 a}$, A.J. Barr ${ }^{118}$, F. Barreiro ${ }^{80}$, J. Barreiro Guimarães da Costa ${ }^{57}$, P. Barrillon ${ }^{115}$, R. Bartoldus ${ }^{143}$, D. Bartsch ${ }^{20}$, R.L. Bates ${ }^{53}$, L. Batkova ${ }^{144 a}$, J.R. Batley ${ }^{27}$, A. Battaglia ${ }^{16}$, M. Battistin ${ }^{29}$, F. Bauer ${ }^{136}$, H.S. Bawa ${ }^{143}$, B. Beare ${ }^{158}$, T. Beau ${ }^{78}$, P.H. Beauchemin ${ }^{118}$, R. Beccherle ${ }^{50 a}$, P. Bechtle ${ }^{41}$, G.A. Beck ${ }^{75}$, H.P. Beck ${ }^{16}$, M. Beckingham ${ }^{48}$, K.H. Becks ${ }^{174}$, A.J. Beddall ${ }^{18 c}$, A. Beddall ${ }^{18 c}$, V.A. Bednyakov ${ }^{65}$, C. Bee $^{83}$, M. Begel ${ }^{24}$, S. Behar Harpaz $^{152}$, P.K. Behera ${ }^{63}$, M. Beimforde ${ }^{99}$, C. Belanger-Champagne ${ }^{166}$, P.J. Bell ${ }^{49}$, W.H. Bell ${ }^{49}$, G. Bella ${ }^{153}$, L. Bellagamba $^{19 a}$, F. Bellina ${ }^{29}$, M. Bellomo ${ }^{119 a}$, A. Belloni ${ }^{57}$, K. Belotskiy ${ }^{96}$, O. Beltramello ${ }^{29}$, S. Ben Ami ${ }^{152}$, O. Benary ${ }^{153}$, D. Benchekroun ${ }^{135 a}$, M. Bendel ${ }^{81}$, B.H. Benedict ${ }^{163}$, N. Benekos ${ }^{165}$, Y. Benhammou ${ }^{153}$, D.P. Benjamin ${ }^{44}$, M. Benoit ${ }^{115}$, J.R. Bensinger ${ }^{22}$, K. Benslama ${ }^{130}$, S. Bentvelsen ${ }^{105}$, M. Beretta ${ }^{47}$, D. Berge ${ }^{29}$, E. Bergeaas Kuutmann ${ }^{41}$, N. Berger ${ }^{4}$, F. Berghaus ${ }^{169}$, E. Berglund ${ }^{49}$, J. Beringer ${ }^{14}$, P. Bernat ${ }^{115}$, R. Bernhard ${ }^{48}$, C. Bernius ${ }^{77}$, T. Berry ${ }^{76}$, A. Bertin ${ }^{19 a, 19 b}$, M.I. Besana ${ }^{89 a, 89 b}$, N. Besson ${ }^{136}$, S. Bethke ${ }^{99}$, R.M. Bianchi ${ }^{48}$, M. Bianco ${ }^{72 a, 72 b}$, O. Biebel ${ }^{98}$, J. Biesiada ${ }^{14}$, M. Biglietti $^{132 a, 132 b}$, H. Bilokon ${ }^{47}$, M. Bindi ${ }^{19 a, 19 b}$, A. Bingul ${ }^{18 c}$, C. Bini ${ }^{132 a, 132 b}$, C. Biscarat ${ }^{180}$, U. Bitenc ${ }^{48}$, K.M. Black ${ }^{57}$, R.E. Blair ${ }^{5}$, J.-B. Blanchard ${ }^{115}$, G. Blanchot ${ }^{29}$, C. Blocker ${ }^{22}$, A. Blondel ${ }^{49}$, W. Blum ${ }^{81}$, U. Blumenschein ${ }^{54}$, G.J. Bobbink $^{105}$, A. Bocci ${ }^{44}$, M. Boehler ${ }^{41}$, J. Boek ${ }^{174}$, N. Boelaert ${ }^{79}$, S. Böser ${ }^{77}$, J.A. Bogaerts ${ }^{29}$, A. Bogouch ${ }^{90}{ }^{*}$, C. Bohm $^{146 a}$, V. Boisvert ${ }^{76}$, T. Bold ${ }^{163, f}$, V. Boldea ${ }^{25 a}$, M. Bondioli ${ }^{163}$, M. Boonekamp ${ }^{136}$, S. Bordoni ${ }^{78}$, C. Borer ${ }^{16}$, A. Borisov ${ }^{128}$, G. Borissov ${ }^{71}$, I. Borjanovic ${ }^{12 a}$, S. Borroni ${ }^{132 a, 132 b}$, K. Bos ${ }^{105}$, D. Boscherini ${ }^{19 a}$, M. Bosman ${ }^{11}$, H. Boterenbrood ${ }^{105}$, J. Bouchami ${ }^{93}$, J. Boudreau ${ }^{123}$, E.V. Bouhova-Thacker ${ }^{71}$, C. Boulahouache ${ }^{123}$, C. Bourdarios ${ }^{115}$, A. Boveia ${ }^{30}$, J. Boyd ${ }^{29}$, 
I.R. Boyko ${ }^{65}$, I. Bozovic-Jelisavcic ${ }^{12 b}$, J. Bracinik ${ }^{17}$, A. Braem ${ }^{29}$, P. Branchini ${ }^{134 a}$, A. Brandt ${ }^{7}$, G. Brandt ${ }^{41}$, O. Brandt ${ }^{54}$, U. Bratzler ${ }^{156}$, B. Brau ${ }^{84}$, J.E. Brau ${ }^{14}$, H.M. Braun ${ }^{174}$, B. Brelier ${ }^{158}$, J. Bremer ${ }^{29}$, R. Brenner ${ }^{166}$, S. Bressler ${ }^{152}$, D. Britton $^{53}$, F.M. Brochu ${ }^{27}$, I. Brock ${ }^{20}$, R. Brock ${ }^{88}$, E. Brodet ${ }^{153}$, G. Brooijmans ${ }^{34}$, W.K. Brooks ${ }^{31 b}$, G. Brown ${ }^{82}$, P.A. Bruckman de Renstrom ${ }^{38}$, D. Bruncko ${ }^{144 b}$, R. Bruneliere ${ }^{48}$, S. Brunet ${ }^{41}$, A. Bruni ${ }^{19 a}$, G. Bruni ${ }^{19 a}$, M. Bruschi ${ }^{19 a}$, F. Bucci ${ }^{49}$, J. Buchanan ${ }^{118}$, P. Buchholz ${ }^{141}$, A.G. Buckley ${ }^{45}$, I.A. Budagov ${ }^{65}$, B. Budick ${ }^{108}$, V. Büscher ${ }^{81}$, L. Bugge ${ }^{117}$, O. Bulekov ${ }^{96}$, M. Bunse ${ }^{42}$, T. Buran ${ }^{117}$, H. Burckhart ${ }^{29}$, S. Burdin ${ }^{73}$, T. Burgess ${ }^{13}$, S. Burke ${ }^{129}$, E. Busato ${ }^{33}$, P. Bussey ${ }^{53}$, C.P. Buszello ${ }^{166}$, F. Butin ${ }^{29}$, B. Butler ${ }^{143}$, J.M. Butler ${ }^{21}$, C.M. Buttar ${ }^{33}$, J.M. Butterworth ${ }^{77}$, T. Byatt ${ }^{77}$, J. Caballero ${ }^{24}$, S. Cabrera Urbán ${ }^{167}$, D. Caforio ${ }^{19 a}$,19b, O. Cakir ${ }^{3 a}$, P. Calafiura ${ }^{14}$, G. Calderini ${ }^{78}$, P. Calfayan ${ }^{98}$, R. Calkins ${ }^{106}$, L.P. Caloba ${ }^{23 a}$, D. Calvet $^{33}$, P. Camarri ${ }^{133 a, 133 b}$, D. Cameron ${ }^{117}$, S. Campana ${ }^{29}$, M. Campanelli77, V. Canale ${ }^{102 a, 102 b}$, F. Canelli ${ }^{30}$, A. Canepa ${ }^{159 a}$, J. Cantero ${ }^{80}$, L. Capasso ${ }^{102 a, 102 b}$, M.D.M. Capeans Garrido ${ }^{29}$, I. Caprini ${ }^{25 a}$, M. Caprini ${ }^{25 a}$, M. Capua ${ }^{36 a, 36 b}$, R. Caputo ${ }^{148}$, C. Caramarcu ${ }^{25 a}$, R. Cardarellii ${ }^{133 a}$, T. Carli ${ }^{29}$, G. Carlino ${ }^{102 a}$, L. Carminati ${ }^{89 a, 89 b}$, B. Caron ${ }^{2, g}$, S. Caron ${ }^{48}$, G.D. Carrillo Montoya ${ }^{172}$, S. Carron Montero ${ }^{158}$, A.A. Carter $^{75}$, J.R. Carter ${ }^{27}$, J. Carvalho ${ }^{124 a, h}$, D. Casadei ${ }^{108}$, M.P. Casado ${ }^{11}$, M. Cascella ${ }^{122 a, 122 b}$, A.M. Castaneda Hernandez ${ }^{172}$, E. Castaneda-Miranda ${ }^{172}$, V. Castillo Gimenez ${ }^{167}$, N.F. Castro ${ }^{124 b, a}$, G. Cataldi ${ }^{72 a}$, A. Catinaccio ${ }^{29}$, J.R. Catmore ${ }^{71}$, A. Cattai ${ }^{29}$, G. Cattani ${ }^{133 a, 133 b}$, S. Caughron ${ }^{34}$, P. Cavalleri ${ }^{78}$, D. Cavalli ${ }^{89 a}$, M. Cavalli-Sforza ${ }^{11}$, V. Cavasinni ${ }^{122 a, 122 b}$, F. Ceradini ${ }^{134 a, 134 b}$, A.S. Cerqueira ${ }^{23 a}$, A. Cerri ${ }^{29}$, L. Cerrito ${ }^{75}$, F. Cerutti ${ }^{47}$, S.A. Cetin ${ }^{18 b}$, A. Chafaq ${ }^{135 a}$, D. Chakraborty ${ }^{106}$, K. Chan $^{2}$, J.D. Chapman ${ }^{27}$, J.W. Chapman ${ }^{87}$, E. Chareyre ${ }^{78}$, D.G. Charlton $^{17}$, V. Chavda ${ }^{82}$, S. Cheatham ${ }^{71}$, S. Chekanov ${ }^{5}$, S.V. Chekulaev ${ }^{159 a}$, G.A. Chelkov ${ }^{65}$, H. Chen ${ }^{24}$, S. Chen ${ }^{32 c}$, X. Chen ${ }^{172}$, A. Cheplakov ${ }^{65}$, V.F. Chepurnov ${ }^{65}$, R. Cherkaoui El Moursli ${ }^{135 d}$, V. Tcherniatine ${ }^{24}$, D. Chesneanu ${ }^{25 a}$, E. Cheu ${ }^{6}$, S.L. Cheung $^{158}$, L. Chevalier ${ }^{136}$, F. Chevallier ${ }^{136}$, G. Chiefari ${ }^{102 a, 102 b}$, L. Chikovani ${ }^{51}$, J.T. Childers ${ }^{58 a}$, A. Chilingarov ${ }^{71}$, G. Chiodini $^{72 a}$, M.V. Chizhov ${ }^{65}$, G. Choudalakis ${ }^{30}$, S. Chouridou ${ }^{137}$, I.A. Christidi ${ }^{77}$, A. Christov ${ }^{48}$, D. Chromek-Burckhart ${ }^{29}$, M.L. Chu ${ }^{151}$, J. Chudoba ${ }^{125}$, G. Ciapetti ${ }^{132 a, 132 b}$, A.K. Ciftci ${ }^{3 a}$, R. Ciftci ${ }^{3 a}$, D. Cinca ${ }^{33}$, V. Cindro ${ }^{74}$, M.D. Ciobotaru ${ }^{163}$, C. Ciocca ${ }^{19 a, 19 b}$, A. Ciocio ${ }^{14}$, M. Cirilli ${ }^{87, i}$, A. Clark $^{49}$, P.J. Clark ${ }^{45}$, W. Cleland ${ }^{123}$, J.C. Clemens ${ }^{83}$, B. Clement ${ }^{55}$, C. Clement ${ }^{146 a, 146 b}$, Y. Coadou ${ }^{83}$, M. Cobal ${ }^{164 a, 164 c}$, A. Coccaro ${ }^{50 a, 50 b}$, J. Cochran ${ }^{64}$, J. Coggeshall ${ }^{165}$, E. Cogneras ${ }^{180}$, A.P. Colijn ${ }^{105}$, C. Collard ${ }^{115}$, N.J. Collins ${ }^{17}$, C. Collins-Tooth ${ }^{53}$, J. Collot ${ }^{55}$, G. Colon ${ }^{84}$, P. Conde Muiño ${ }^{124 a}$, E. Coniavitis $^{166}$, M.C. Conidi ${ }^{11}$, M. Consonni ${ }^{104}$, S. Constantinescu ${ }^{25 a}$, C. Conta ${ }^{119 a, 119 b}$, F. Conventi102a,j, M. Cooke ${ }^{34}$, B.D. Cooper ${ }^{75}$, A.M. Cooper-Sarkar ${ }^{118}$, N.J. Cooper-Smith ${ }^{76}$, K. Copic ${ }^{34}$, T. Cornelissen ${ }^{50 a, 50 b}$, M. Corradi ${ }^{19 a}$, F. Corriveau $^{85, \mathrm{k}}$, A. Corso-Radu ${ }^{163}$, A. Cortes-Gonzalez ${ }^{165}$, G. Cortiana ${ }^{99}$, G. Costa ${ }^{89 a}$, M.J. Costa ${ }^{167}$, D. Costanzo ${ }^{139}$, T. Costin $^{30}$, D. Côté ${ }^{29}$, R. Coura Torres ${ }^{23 a}$, L. Courneyea ${ }^{169}$, G. Cowan $^{76}$, C. Cowden ${ }^{27}$, B.E. Cox ${ }^{82}$, K. Cranmer ${ }^{108}$, J. Cranshaw ${ }^{5}$, M. Cristinziani ${ }^{20}$, G. Crosetti ${ }^{36 a, 36 b}$, R. Crupi ${ }^{72 a, 72 b}$, S. Crépé-Renaudin ${ }^{55}$, C. Cuenca Almenar ${ }^{175}$, T. Cuhadar Donszelmann $^{139}$, M. Curatolo ${ }^{47}$, C.J. Curtis ${ }^{17}$, P. Cwetanski ${ }^{61}$, Z. Czyczula ${ }^{175}$, S. D'Auria ${ }^{53}$, M. D'Onofrio ${ }^{73}$, A. D'Orazio ${ }^{99}$, C. Da Via ${ }^{82}$, W. Dabrowski ${ }^{37}$, T. Dai ${ }^{77}$, C. Dallapiccola ${ }^{84}$, S.J. Dallison ${ }^{129, *}$, C.H. Daly ${ }^{138}$, M. Dam ${ }^{35}$, H.O. Danielsson $^{29}$, D. Dannheim ${ }^{99}$, V. Dao ${ }^{49}$, G. Darbo ${ }^{50 a}$, G.L. Darlea ${ }^{25 b}$, W. Davey ${ }^{86}$, T. Davidek ${ }^{126}$, N. Davidson ${ }^{86}$, R. Davidson ${ }^{71}$, M. Davies ${ }^{93}$, A.R. Davison ${ }^{77}$, I. Dawson ${ }^{139}$, R.K. Daya ${ }^{39}$, K. De $^{7}$, R. de Asmundis ${ }^{102 a}$, S. De Castro ${ }^{19 a}, 19 b$, P.E. De Castro Faria Salgado ${ }^{24}$, S. De $\mathrm{Cecco}^{78}$, J. de Graat ${ }^{98}$, N. De Groot ${ }^{104}$, P. de Jong ${ }^{105}$, L. De Mora ${ }^{71}$, M. De Oliveira Branco ${ }^{29}$, D. De Pedis ${ }^{132 a}$, A. De Salvo ${ }^{132 a}$, U. De Sanctis ${ }^{164 a, 164 c}$, A. De Santo ${ }^{149}$, J.B. De Vivie De Regie ${ }^{115}$, S. Dean ${ }^{77}$, D.V. Dedovich $^{65}$, J. Degenhardt ${ }^{120}$, M. Dehchar ${ }^{118}$, C. Del Papa ${ }^{164 a, 164 c}$, J. Del Peso ${ }^{80}$, T. Del Prete ${ }^{122 a, 122 b}$, A. Dell'Acqua ${ }^{29}$, L. Dell'Asta ${ }^{89 a, 89 b}$, M. Della Pietra ${ }^{102 a, 1}$, D. della Volpe ${ }^{102 a, 102 b}$, M. Delmastro ${ }^{29}$, P.A. Delsart ${ }^{55}$, C. Deluca ${ }^{148}$, S. Demers $^{175}$, M. Demichev ${ }^{65}$, B. Demirkoz ${ }^{11}$, J. Deng ${ }^{163}$, W. Deng ${ }^{24}$, S.P. Denisov ${ }^{128}$, J.E. Derkaoui ${ }^{135 c}$, F. Derue ${ }^{78}$, P. Dervan ${ }^{73}$, K. Desch ${ }^{20}$, P.O. Deviveiros ${ }^{158}$, A. Dewhurst ${ }^{129}$, B. DeWilde ${ }^{148}$, S. Dhaliwal ${ }^{158}$, R. Dhullipudi ${ }^{24, m}$, A. Di Ciaccio ${ }^{133 a, 133 b}$, L. Di $\mathrm{Ciaccio}^{4}$, A. Di Girolamo ${ }^{29}$, B. Di Girolamo ${ }^{29}$, S. Di Luise ${ }^{134 a, 134 b}$, A. Di Mattia ${ }^{88}$, R. Di Nardo ${ }^{133 a, 133 b}$, A. Di Simone ${ }^{133 a, 133 b}$, R. Di Sipio ${ }^{19 a, 19 b}$, M.A. Diaz ${ }^{31 a}$, F. Diblen ${ }^{18 c}$, E.B. Diehl ${ }^{87}$, J. Dietrich ${ }^{48}$, T.A. Dietzsch ${ }^{58 a}$, S. Diglio ${ }^{115}$, K. Dindar Yagci ${ }^{39}$, J. Dingfelder ${ }^{48}$, C. Dionisi ${ }^{132 a, 132 b}$, P. Dita ${ }^{25 a}$, S. Dita ${ }^{25 a}$, F. Dittus ${ }^{29}$, F. Djama ${ }^{83}$, R. Djilkibaev ${ }^{108}$, T. Djobava ${ }^{51}$, M.A.B. do Vale ${ }^{23 a}$, T.K.O. Doan ${ }^{4}$, D. Dobos $^{29}$, E. Dobson ${ }^{29}$, M. Dobson ${ }^{163}$, C. Doglioni ${ }^{118}$, T. Doherty ${ }^{53}$, J. Dolejsi ${ }^{126}$, I. Dolenc ${ }^{74}$, Z. Dolezal ${ }^{126}$, B.A. Dolgoshein ${ }^{96}$, T. Dohmae ${ }^{155}$, M. Donega ${ }^{120}$, J. Donini ${ }^{55}$, J. Dopke ${ }^{174}$, A. Doria ${ }^{102 a}$, A. Dotti ${ }^{122 a, 122 b}$, M.T. Dova ${ }^{70}$, A.D. Doxiadis ${ }^{105}$, A.T. Doyle ${ }^{53}$, Z. Drasal ${ }^{126}$, M. Dris ${ }^{9}$, J. Dubbert ${ }^{99}$, S. Dube ${ }^{14}$, E. Duchovni ${ }^{171}$, G. Duckeck ${ }^{98}$, A. Dudarev ${ }^{29}$, F. Dudziak ${ }^{115}$, M. Dührssen ${ }^{29}$, L. Duflot ${ }^{115}$, M.-A. Dufour ${ }^{85}$,

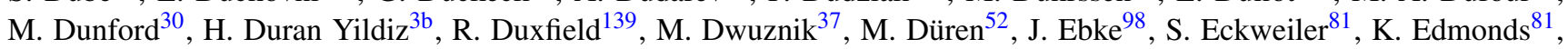
C.A. Edwards ${ }^{76}$, K. Egorov $^{61}$, W. Ehrenfeld ${ }^{41}$, T. Ehrich ${ }^{99}$, T. Eifert ${ }^{29}$, G. Eigen ${ }^{13}$, K. Einsweiler ${ }^{14}$, E. Eisenhandler ${ }^{75}$, T. Ekelof ${ }^{166}$, M. El Kacimi ${ }^{4}$, M. Ellert ${ }^{166}$, S. Elles ${ }^{4}$, F. Ellinghaus ${ }^{81}$, K. Ellis ${ }^{75}$, N. Ellis ${ }^{29}$, J. Elmsheuser ${ }^{98}$, M. Elsing ${ }^{29}$, D. Emeliyanov ${ }^{129}$, R. Engelmann 148 , A. Engl ${ }^{98}$, B. Epp $^{62}$, A. Eppig ${ }^{87}$, J. Erdmann ${ }^{54}$, A. Ereditato ${ }^{16}$, D. Eriksson ${ }^{146 a}$, J. Ernst ${ }^{1}$, M. Ernst ${ }^{24}$, J. Ernwein ${ }^{136}$, D. Errede ${ }^{165}$, S. Errede ${ }^{165}$, E. Ertel ${ }^{81}$, M. Escalier ${ }^{115}$, C. Escobar ${ }^{167}$, X. Espinal Curull $^{11}$, B. Esposito ${ }^{47}$, A.I. Etienvre ${ }^{136}$, E. Etzion ${ }^{153}$, H. Evans ${ }^{61}$, L. Fabbri ${ }^{19 a}$,19b , C. Fabre ${ }^{29}$, K. Facius ${ }^{35}$, R.M. Fakhrutdinov $^{128}$, S. Falciano ${ }^{132 a}$, Y. Fang ${ }^{172}$, M. Fanti ${ }^{89 a}$,89b, A. Farbin ${ }^{7}$, A. Farilla ${ }^{134 a}$, J. Farley ${ }^{148}$, T. Farooque ${ }^{158}$, S.M. Farrington $^{118}$, P. Farthouat ${ }^{29}$, P. Fassnacht ${ }^{29}$, D. Fassouliotis ${ }^{8}$, B. Fatholahzadeh ${ }^{158}$, L. Fayard ${ }^{115}$, R. Febbraro ${ }^{33}$, P. Federic ${ }^{144 a}$, 
O.L. Fedin ${ }^{121}$, W. Fedorko ${ }^{29}$, L. Feligioni ${ }^{83}$, C.U. Felzmann ${ }^{86}$, C. Feng ${ }^{32 d}$, E.J. Feng ${ }^{30}$, A.B. Fenyuk ${ }^{128}$, J. Ferencei ${ }^{144 b}$, J. Ferland ${ }^{93}$, B. Fernandes ${ }^{124 a, n}$, W. Fernando ${ }^{109}$, S. Ferrag ${ }^{53}$, J. Ferrando ${ }^{118}$, V. Ferrara ${ }^{41}$, A. Ferrari ${ }^{166}$, P. Ferrari ${ }^{105}$, R. Ferrari $^{119 a}$, A. Ferrer ${ }^{167}$, M.L. Ferrer ${ }^{47}$, D. Ferrere ${ }^{49}$, C. Ferretti ${ }^{87}$, M. Fiascaris ${ }^{118}$, F. Fiedler ${ }^{81}$, A. Filipčič ${ }^{74}$, A. Filippas ${ }^{9}$, F. Filthaut ${ }^{104}$, M. Fincke-Keeler ${ }^{169}$, M.C.N. Fiolhais ${ }^{124 a, h}$, L. Fiorini ${ }^{11}$, A. Firan ${ }^{39}$, G. Fischer ${ }^{41}$, M.J. Fisher ${ }^{109}$, M. Flechl ${ }^{48}$, I. Fleck ${ }^{141}$, J. Fleckner ${ }^{81}$, P. Fleischmann ${ }^{173}$, S. Fleischmann ${ }^{20}$, T. Flick ${ }^{174}$, L.R. Flores Castillo ${ }^{172}$, M.J. Flowerdew ${ }^{99}$, T. Fonseca Martin ${ }^{76}$, J. Fopma ${ }^{118}$, A. Formica ${ }^{136}$, A. Forti ${ }^{82}$, D. Fortin ${ }^{159 a}$, D. Fournier ${ }^{115}$, A.J. Fowler ${ }^{44}$, K. Fowler ${ }^{137}$, H. Fox ${ }^{71}$, P. Francavilla ${ }^{122 a, 122 b}$, S. Franchino ${ }^{119 a, 119 b}$, D. Francis ${ }^{29}$, M. Franklin ${ }^{57}$, S. Franz ${ }^{29}$, M. Fraternali ${ }^{119 a, 119 b}$, S. Fratina ${ }^{120}$, J. Freestone ${ }^{82}$, S.T. French ${ }^{27}$, R. Froeschl ${ }^{29}$, D. Froidevaux ${ }^{29}$, J.A. Frost ${ }^{27}$, C. Fukunaga ${ }^{156}$, E. Fullana Torregrosa $^{5}$, J. Fuster ${ }^{167}$, C. Gabaldon ${ }^{80}$, O. Gabizon ${ }^{171}$, T. Gadfort ${ }^{24}$, S. Gadomski ${ }^{49}$, G. Gagliardi ${ }^{50 a, 50 b}$, P. Gagnon ${ }^{61}$, C. Galea ${ }^{98}$, E.J. Gallas ${ }^{118}$, V. Gallo ${ }^{16}$, B.J. Gallop ${ }^{129}$, P. Gallus ${ }^{125, o}$, E. Galyaev ${ }^{40}$, K.K. Gan ${ }^{109}$, Y.S. Gao ${ }^{143, \text { p }, ~ A . ~ G a p o-~}$ nenko ${ }^{14}$, M. Garcia-Sciveres ${ }^{14}$, C. García ${ }^{167}$, J.E. García Navarro ${ }^{49}$, R.W. Gardner ${ }^{30}$, N. Garelli ${ }^{29}$, H. Garitaonandia ${ }^{105}$, V. Garonne ${ }^{29}$, C. Gatti ${ }^{47}$, G. Gaudio ${ }^{119 a}$, P. Gauzzi ${ }^{132 a, 132 b}$, I.L. Gavrilenko ${ }^{94}$, C. Gay ${ }^{168}$, G. Gaycken ${ }^{20}$, E.N. Gazis ${ }^{9}$, P. Ge ${ }^{32 d}$, C.N.P. Gee ${ }^{129}$, Ch. Geich-Gimbel ${ }^{20}$, K. Gellerstedt ${ }^{146 a, 146 b}$, C. Gemme ${ }^{50 a}$, M.H. Genest ${ }^{98}$, S. Gentile ${ }^{132 a, 132 b}$, F. Georgatos ${ }^{9}$, S. George ${ }^{76}$, A. Gershon ${ }^{153}$, H. Ghazlane ${ }^{135 d}$, N. Ghodbane ${ }^{33}$, B. Giacobbe ${ }^{19 a}$, S. Giagu ${ }^{132 a, 132 b}$, V. Giakoumopoulou $^{8}$, V. Giangiobbe ${ }^{122 a, 122 b}$, F. Gianotti ${ }^{29}$, B. Gibbard ${ }^{24}$, A. Gibson ${ }^{158}$, S.M. Gibson ${ }^{118}$, L.M. Gilbert ${ }^{118}$, M. Gilchriese ${ }^{14}$, V. Gilewsky ${ }^{91}$, D.M. Gingrich ${ }^{2, \mathrm{q}}$, J. Ginzburg ${ }^{153}$, N. Giokaris ${ }^{8}$, M.P. Giordani ${ }^{164 \mathrm{c}}$, R. Giordano ${ }^{102 a, 102 \mathrm{~b}}$, F.M. Giorgi ${ }^{15}$, P. Giovannini ${ }^{99}$, P.F. Giraud ${ }^{136}$, D. Giugni ${ }^{89 a}$, P. Giusti ${ }^{19 a}$, B.K. Gjelsten ${ }^{117}$, L.K. Gladilin ${ }^{97}$, C. Glasman ${ }^{80}$, A. Glazov ${ }^{41}$, K.W. Glitza ${ }^{174}$, G.L. Glonti ${ }^{65}$, J. Godfrey ${ }^{142}$, J. Godlewski ${ }^{29}$, M. Goebel ${ }^{41}$, T. Göpfert ${ }^{43}$, C. Goeringer ${ }^{81}$, C. Gössling ${ }^{42}$, T. Göttfert ${ }^{99}$, S. Goldfarb ${ }^{87}$, D. Goldin ${ }^{39}$, T. Golling ${ }^{175}$, A. Gomes ${ }^{124 a, r}$, L.S. Gomez Fajardo ${ }^{41}$, R. Gonçalo ${ }^{76}$, L. Gonella ${ }^{20}$, C. Gong ${ }^{32 b}$, S. González de la $\mathrm{Hoz}^{167}$, M.L. Gonzalez Silva ${ }^{26}$, S. Gonzalez-Sevilla ${ }^{49}$, J.J. Goodson ${ }^{148}$, L. Goossens ${ }^{29}$, H.A. Gordon ${ }^{24}$, I. Gorelov ${ }^{103}$, G. Gorfine ${ }^{174}$, B. Gorini ${ }^{29}$, E. Gorini ${ }^{72 a, 72 b}$, A. Gorišek ${ }^{74}$, E. Gornicki ${ }^{38}$, B. Gosdzik ${ }^{41}$, M. Gosselink ${ }^{105}$, M.I. Gostkin ${ }^{65}$, I. Gough Eschrich ${ }^{163}$, M. Gouighri ${ }^{135 a}$, D. Goujdami ${ }^{135 a}$, M.P. Goulette ${ }^{49}$, A.G. Goussiou ${ }^{138}$, C. Goy ${ }^{4}$, I. Grabowska-Bold ${ }^{163, s}$, P. Grafström ${ }^{29}$, K.-J. Grahn ${ }^{147}$, S. Grancagnolo ${ }^{15}$, V. Grassi ${ }^{148}$, V. Gratchev ${ }^{121}$, N. Grau ${ }^{34}$, H.M. Gray ${ }^{34, t}$, J.A. Gray ${ }^{148}$, E. Graziani ${ }^{134 a}$, B. Green ${ }^{76}$, T. Greenshaw ${ }^{73}$, Z.D. Greenwood ${ }^{24, u}$, I.M. Gregor ${ }^{41}$, P. Grenier ${ }^{143}$, E. Griesmayer ${ }^{46}$, J. Griffiths ${ }^{138}$, N. Grigalashvili65, A.A. Grillo ${ }^{137}$, K. Grimm ${ }^{148}$, S. Grinstein $^{11}$, Y.V. Grishkevich ${ }^{97}$, M. Groh ${ }^{99}$, M. Groll ${ }^{81}$, E. Gross ${ }^{171}$, J. Grosse-Knetter ${ }^{54}$, J. Groth-Jensen ${ }^{79}$, K. Grybel ${ }^{141}$, C. Guicheney ${ }^{33}$, A. Guida ${ }^{72 a, 72 b}$, T. Guillemin ${ }^{4}$, H. Guler ${ }^{85, v}$, J. Gunther ${ }^{125}$, B. Guo ${ }^{158}$, Y. Gusakov ${ }^{65}$, A. Gutierrez ${ }^{93}$, P. Gutierrez ${ }^{111}$, N. Guttman ${ }^{153}$, O. Gutzwiller ${ }^{172}$, C. Guyot ${ }^{136}$, C. Gwenlan ${ }^{118}$, C.B. Gwilliam ${ }^{73}$, A. Haas ${ }^{143}$, S. Haas ${ }^{29}$, C. Haber ${ }^{14}$, H.K. Hadavand ${ }^{39}$, D.R. Hadley ${ }^{17}$, P. Haefner ${ }^{99}$, S. Haider ${ }^{29}$, Z. Hajduk ${ }^{38}$, H. Hakobyan ${ }^{176}$, J. Haller ${ }^{41, w}$, K. Hamacher ${ }^{174}$, A. Hamilton ${ }^{49}$, S. Hamilton ${ }^{161}$, L. Han ${ }^{32 b}$, K. Hanagaki ${ }^{116}$, M. Hance ${ }^{120}$, C. Handel ${ }^{81}$, P. Hanke ${ }^{58 a}$, J.R. Hansen ${ }^{35}$, J.B. Hansen ${ }^{35}$, J.D. Hansen ${ }^{35}$, P.H. Hansen ${ }^{35}$, P. Hansson ${ }^{143}$, K. Hara ${ }^{160}$, G.A. Hare ${ }^{137}$, T. Harenberg $^{174}$, R.D. Harrington ${ }^{21}$, O.M. Harris ${ }^{138}$, K. Harrison ${ }^{17}$, J. Hartert ${ }^{48}$, F. Hartjes ${ }^{105}$, A. Harvey ${ }^{56}$, S. Hasegawa ${ }^{101}$, Y. Hasegawa ${ }^{140}$, S. Hassani ${ }^{136}$, S. Haug ${ }^{16}$, M. Hauschild ${ }^{29}$, R. Hauser ${ }^{88}$, M. Havranek ${ }^{125, o}$, C.M. Hawkes ${ }^{17}$, R.J. Hawkings $^{29}$, T. Hayakawa ${ }^{67}$, H.S. Hayward ${ }^{73}$, S.J. Haywood ${ }^{129}$, S.J. Head ${ }^{17}$, V. Hedberg ${ }^{79}$, L. Heelan ${ }^{28}$, S. Heim ${ }^{88}$, B. Heinemann $^{14}$, S. Heisterkamp ${ }^{35}$, L. Helary ${ }^{4}$, M. Heller ${ }^{15}$, S. Hellman ${ }^{146 a, 146 b}$, C. Helsens ${ }^{11}$, T. Hemperek ${ }^{20}$, R.C.W. Hender$\operatorname{son}^{71}$, M. Henke ${ }^{58 a}$, A. Henrichs ${ }^{54}$, A.M. Henriques Correia ${ }^{29}$, S. Henrot-Versille ${ }^{115}$, C. Hensel ${ }^{54}$, T. Henß ${ }^{174}$, Y. Hernández Jiménez $^{167}$, A.D. Hershenhorn ${ }^{152}$, G. Herten ${ }^{48}$, R. Hertenberger ${ }^{98}$, L. Hervas ${ }^{29}$, N.P. Hessey ${ }^{105}$, E. Higón-Rodriguez ${ }^{167}$, J.C. Hill ${ }^{27}$, K.H. Hiller ${ }^{41}$, S. Hillert ${ }^{146 a, 146 b}$, S.J. Hillier ${ }^{17}$, I. Hinchliffe ${ }^{14}$, E. Hines ${ }^{120}$, M. Hirose ${ }^{116}$, F. Hirsch ${ }^{42}$, D. Hirschbuehl ${ }^{174}$, J. Hobbs ${ }^{148}$, N. Hod ${ }^{153}$, M.C. Hodgkinson ${ }^{139}$, P. Hodgson ${ }^{139}$, A. Hoecker ${ }^{29}$, M.R. Hoeferkamp ${ }^{103}$, J. Hoffman ${ }^{39}$, D. Hoffmann ${ }^{83}$, M. Hohlfeld ${ }^{81}$, T. Holy ${ }^{127}$, J.L. Holzbauer ${ }^{88}$, Y. Homma ${ }^{67}$, T. Horazdovsky ${ }^{127}$, C. Horn ${ }^{143}$, S. Horner ${ }^{48}$, J.-Y. Hostachy ${ }^{55}$, S. Hou ${ }^{151}$, A. Hoummada ${ }^{135 a}$, T. Howe ${ }^{39}$, J. Hrivnac ${ }^{115}$, T. Hryn'ova ${ }^{4}$, P.J. Hsu ${ }^{175}$,

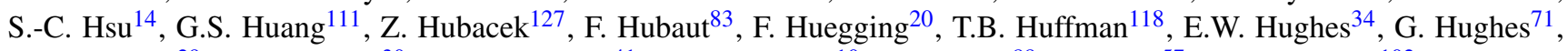
M. Huhtinen ${ }^{29}$, M. Hurwitz ${ }^{30}$, U. Husemann ${ }^{41}$, N. Huseynov ${ }^{10}$, J. Huston ${ }^{88}$, J. Huth ${ }^{57}$, G. Iacobucci ${ }^{102 a}$, G. Iakovidis $^{9}$, I. Ibragimov ${ }^{141}$, L. Iconomidou-Fayard ${ }^{115}$, J. Idarraga ${ }^{159 b}$, P. Iengo ${ }^{4}$, O. Igonkina ${ }^{105}$, Y. Ikegami ${ }^{66}$, M. Ikeno ${ }^{66}$, Y. Ilchenko $^{39}$, D. Iliadis ${ }^{154}$, T. Ince ${ }^{20}$, P. Ioannou ${ }^{8}$, M. Iodice $^{134 a}$, A. Irles Quiles ${ }^{167}$, A. Ishikawa ${ }^{67}$, M. Ishino $^{66}$, R. Ishmukhametov $^{39}$, T. Isobe ${ }^{155}$, C. Issever ${ }^{118}$, S. Istin ${ }^{18 a}$, Y. Itoh ${ }^{101}$, A.V. Ivashin ${ }^{128}$, W. Iwanski ${ }^{38}$, H. Iwasaki ${ }^{66}$, J.M. Izen $^{40}$, V. Izzo ${ }^{102 a}$, B. Jackson ${ }^{120}$, J.N. Jackson ${ }^{73}$, P. Jackson ${ }^{143}$, M.R. Jaekel ${ }^{29}$, V. Jain ${ }^{61}$, K. Jakobs ${ }^{48}$, S. Jakobsen ${ }^{35}$, J. Jakubek ${ }^{127}$, D.K. Jana ${ }^{111}$, E. Jankowski ${ }^{158}$, E. Jansen ${ }^{77}$, A. Jantsch ${ }^{99}$, M. Janus ${ }^{48}$, G. Jarlskog ${ }^{79}$, L. Jeanty ${ }^{57}$, I. Jen-La Plante ${ }^{30}$, P. Jenni ${ }^{29}$, P. Jež ${ }^{35}$, S. Jézéquel ${ }^{4}$, W. Ji ${ }^{79}$, J. Jia ${ }^{148}$, Y. Jiang ${ }^{32 b}$, M.Jimenez Belenguer ${ }^{29}$, S. Jin ${ }^{32 a}$, O. Jinnouchi ${ }^{157}$, D. Joffe ${ }^{39}$, M. Johansen ${ }^{146 a, 146 b}$, K.E. Johansson ${ }^{146 a}$, P. Johansson ${ }^{139}$, S. Johnert ${ }^{41}$, K.A. Johns ${ }^{6}$, K. Jon-And ${ }^{146 a, 146 b}$, G. Jones ${ }^{82}$, R.W.L. Jones ${ }^{71}$, T.J. Jones ${ }^{73}$, P.M. Jorge ${ }^{124 a, b}$, J. Joseph ${ }^{14}$, V. Juranek ${ }^{125}$, P. Jussel ${ }^{62}$, V.V. Kabachenko ${ }^{128}$, M. Kaci ${ }^{167}$, A. Kaczmarska ${ }^{38}$, M. Kado ${ }^{115}$, H. Kagan ${ }^{109}$, M. Kagan ${ }^{57}$, S. Kaiser ${ }^{99}$, E. Kajomovitz ${ }^{152}$, S. Kalinin ${ }^{174}$, L.V. Kalinovskaya ${ }^{65}$, S. Kama ${ }^{41}$, N. Kanaya ${ }^{155}$, M. Kaneda ${ }^{155}$, V.A. Kantserov ${ }^{96}$, J. Kanzaki ${ }^{66}$, B. Kaplan ${ }^{175}$, A. Kapliy ${ }^{30}$, J. Kaplon ${ }^{29}$, D. Kar ${ }^{43}$, 
M. Karagounis ${ }^{20}$, M. Karagoz ${ }^{118}$, M. Karnevskiy ${ }^{41}$, V. Kartvelishvili ${ }^{71}$, A.N. Karyukhin ${ }^{128}$, L. Kashif ${ }^{57}$, A. Kasmi ${ }^{39}$, R.D. Kass ${ }^{109}$, A. Kastanas ${ }^{13}$, M. Kataoka ${ }^{4}$, Y. Kataoka ${ }^{155}$, E. Katsoufis ${ }^{9}$, J. Katzy ${ }^{41}$, V. Kaushik ${ }^{6}$, K. Kawagoe ${ }^{67}$, T. Kawamoto ${ }^{155}$, G. Kawamura ${ }^{81}$, M.S. Kayl ${ }^{105}$, V.A. Kazanin ${ }^{107}$, M.Y. Kazarinov ${ }^{65}$, J.R. Keates ${ }^{82}$, R. Keeler ${ }^{169}$, R. Kehoe $^{39}$, M. Keil ${ }^{54}$, G.D. Kekelidze ${ }^{65}$, M. Kelly ${ }^{82}$, M. Kenyon ${ }^{53}$, O. Kepka ${ }^{125}$, N. Kerschen ${ }^{29}$, B.P. Kerševan ${ }^{74}$, S. Kersten ${ }^{174}$, K. Kessoku ${ }^{155}$, M. Khakzad ${ }^{28}$, F. Khalil-zada ${ }^{10}$, H. Khandanyan ${ }^{165}$, A. Khanov ${ }^{112}$, D. Kharchenko ${ }^{65}$, A. Khodinov ${ }^{148}$, A. Khomich ${ }^{58 a}$, G. Khoriauli ${ }^{20}$, N. Khovanskiy ${ }^{65}$, V. Khovanskiy ${ }^{95}$, E. Khramov ${ }^{65}$, J. Khubua ${ }^{51}$, H. Kim ${ }^{7}$, M.S. Kim ${ }^{2}$, P.C. $\mathrm{Kim}^{143}$, S.H. Kim ${ }^{160}$, O. Kind ${ }^{15}$, B.T. King ${ }^{73}$, M. King ${ }^{67}$, J. Kirk ${ }^{129}$, G.P. Kirsch ${ }^{118}$, L.E. Kirsch ${ }^{22}$, A.E. Kiryunin ${ }^{99}$, D. Kisielewska ${ }^{37}$, T. Kittelmann ${ }^{123}$, E. Kladiva ${ }^{144 b}$, M. Klein ${ }^{73}$, U. Klein ${ }^{73}$, K. Kleinknecht ${ }^{81}$, M. Klemetti ${ }^{85}$, A. Klier ${ }^{171}$, A. Klimentov ${ }^{24}$, R. Klingenberg ${ }^{42}$, E.B. Klinkby ${ }^{44}$, T. Klioutchnikova ${ }^{29}$, P.F. Klok ${ }^{104}$, S. Klous ${ }^{105}$, E.-E. Kluge ${ }^{58 a}$, T. Kluge ${ }^{73}$, P. Kluit ${ }^{105}$, S. Kluth ${ }^{99}$, N.S. Knecht ${ }^{158}$, E. Kneringer ${ }^{62}$, B.R. Ko ${ }^{44}$, T. Kobayashi ${ }^{155}$, M. Kobel ${ }^{43}$, B. Koblitz ${ }^{29}$, M. Kocian ${ }^{143}$, A. Kocnar ${ }^{113}$, P. Kodys ${ }^{126}$, K. Köneke ${ }^{41}$, A.C. König ${ }^{104}$, S. Koenig ${ }^{81}$, L. Köpke ${ }^{81}$, F. Koetsveld ${ }^{104}$, P. Ko-

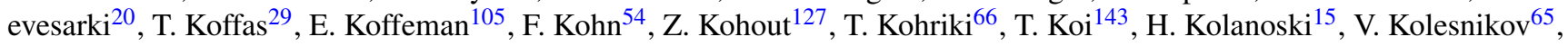
I. Koletsou ${ }^{4}$, J. Koll ${ }^{88}$, D. Kollar ${ }^{29}$, S.D. Kolya ${ }^{82}$, A.A. Komar ${ }^{94}$, J.R. Komaragiri ${ }^{142}$, T. Kondo ${ }^{66}$, T. Kono ${ }^{41, x}$, R. Konoplich $^{108}$, N. Konstantinidis ${ }^{77}$, S. Koperny ${ }^{37}$, K. Korcyl ${ }^{38}$, K. Kordas ${ }^{154}$, A. Korn ${ }^{14}$, I. Korolkov ${ }^{11}$, E.V. Korolkova ${ }^{139}$, V.A. Korotkov ${ }^{128}$, O. Kortner ${ }^{99}$, S. Kortner ${ }^{99}$, P. Kostka ${ }^{41}$, V.V. Kostyukhin ${ }^{20}$, S. Kotov ${ }^{99}$, V.M. Kotov ${ }^{65}$, C. Kourkoumelis ${ }^{8}$, A. Koutsman ${ }^{105}$, R. Kowalewski ${ }^{169}$, T.Z. Kowalski ${ }^{37}$, W. Kozanecki ${ }^{136}$, A.S. Kozhin ${ }^{128}$, V. Kral ${ }^{127}$, V.A. Kramarenko ${ }^{97}$, G. Kramberger ${ }^{74}$, M.W. Krasny ${ }^{78}$, A. Krasznahorkay ${ }^{108}$, J. Kraus ${ }^{88}$, J.K. Kraus ${ }^{20}$, A. Kreisel ${ }^{153}$, F. Krejci ${ }^{127}$, J. Kretzschmar $^{73}$, N. Krieger ${ }^{54}$, P. Krieger ${ }^{158}$, K. Kroeninger ${ }^{54}$, H. Kroha ${ }^{99}$, J. Kroll ${ }^{120}$, J. Kroseberg ${ }^{20}$, J. Krstic ${ }^{12 a}$, U. Kruchonak $^{65}$, H. Krüger ${ }^{20}$, Z.V. Krumshteyn ${ }^{65}$, A. Kruth ${ }^{20}$, T. Kubota ${ }^{155}$, S. Kuehn ${ }^{48}$, A. Kugel ${ }^{58 c}$, T. Kuhl ${ }^{174}$, D. Kuhn ${ }^{62}$, V. Kukhtin ${ }^{65}$, Y. Kulchitsky ${ }^{90}$, S. Kuleshov ${ }^{31 b}$, C. Kummer ${ }^{98}$, M. Kuna ${ }^{83}$, J. Kunkle ${ }^{120}$, A. Kupco ${ }^{125}$, H. Kurashige ${ }^{67}$, M. Kurata $^{160}$, Y.A. Kurochkin ${ }^{90}$, V. Kus ${ }^{125}$, M. Kuze ${ }^{157}$, R. Kwee ${ }^{15}$, A. La Rosa ${ }^{29}$, L. La Rotonda ${ }^{36 a, 36 b}$, J. Labbe ${ }^{4}$, C. Lacasta ${ }^{167}$, F. Lacava ${ }^{132 a, 132 b}$, H. Lacker ${ }^{15}$, D. Lacour ${ }^{78}$, V.R. Lacuesta ${ }^{167}$, E. Ladygin ${ }^{65}$, R. Lafaye ${ }^{4}$, B. Laforge ${ }^{78}$, T. Lagouri ${ }^{80}$,

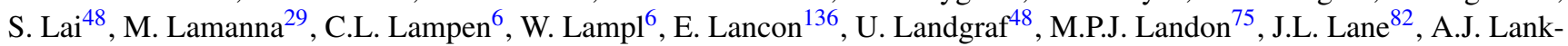
ford ${ }^{163}$, F. Lanni ${ }^{24}$, K. Lantzsch $^{29}$, A. Lanza ${ }^{119 a}$, S. Laplace ${ }^{4}$, C. Lapoire ${ }^{83}$, J.F. Laporte ${ }^{136}$, T. Lari ${ }^{89 a}$, A. Larner ${ }^{118}$, M. Lassnig ${ }^{29}$, P. Laurelli ${ }^{47}$, W. Lavrijsen ${ }^{14}$, P. Laycock ${ }^{73}$, A.B. Lazarev ${ }^{65}$, A. Lazzaro ${ }^{89 a, 89 b}$, O. Le Dortz ${ }^{78}$, E. Le Guirriec $^{83}$, E. Le Menedeu ${ }^{136}$, A. Lebedev ${ }^{64}$, C. Lebel ${ }^{93}$, T. LeCompte ${ }^{5}$, F. Ledroit-Guillon ${ }^{55}$, H. Lee ${ }^{105}$, J.S.H. Lee ${ }^{150}$, S.C. Lee $^{151}$, M. Lefebvre ${ }^{169}$, M. Legendre ${ }^{136}$, B.C. LeGeyt ${ }^{120}$, F. Legger ${ }^{98}$, C. Leggett ${ }^{14}$, M. Lehmacher ${ }^{20}$, G. Lehmann Miotto $^{29}$, X. Lei ${ }^{6}$, R. Leitner ${ }^{126}$, D. Lellouch ${ }^{171}$, J. Lellouch ${ }^{78}$, V. Lendermann ${ }^{58 a}$, K.J.C. Leney ${ }^{73}$, T. Lenz ${ }^{174}$, G. Lenzen ${ }^{174}$, B. Lenzi ${ }^{136}$, K. Leonhardt ${ }^{43}$, C. Leroy ${ }^{93}$, J.-R. Lessard ${ }^{169}$, C.G. Lester ${ }^{27}$, A. Leung Fook Cheong ${ }^{172}$, J. Levêque ${ }^{83}$, D. Levin ${ }^{87}$, L.J. Levinson ${ }^{171}$, M. Leyton ${ }^{15}, \mathrm{H}_{\text {. } \mathrm{Li}^{172}, \mathrm{X}^{1} \mathrm{Li}^{87} \text {, Z. Liang }}{ }^{39}$, Z. Liang ${ }^{151, \mathrm{y}}$, B. Liberti ${ }^{133 a}$, P. Lichard ${ }^{29}$, M. Lichtnecker ${ }^{98}$, K. Lie ${ }^{165}$, W. Liebig ${ }^{105}$, J.N. Lilley ${ }^{17}$, A. Limosani86, M. Limper ${ }^{63}$, S.C. Lin ${ }^{151}$, J.T. Linnemann ${ }^{88}$, E. Lipeles ${ }^{120}$, L. Lipinsky ${ }^{125}$, A. Lipniacka ${ }^{13}$, T.M. Liss ${ }^{165}$, D. Lissauer ${ }^{24}$, A. Lister ${ }^{49}$, A.M. Litke ${ }^{137}$, C. Liu ${ }^{28}$, D. Liu ${ }^{151, z}$, H. Liu ${ }^{87}$, J.B. Liu ${ }^{87}$, M. Liu ${ }^{32 b}$, Y. Liu ${ }^{32 b}$, M. Livan ${ }^{119 a, 119 b}$, A. Lleres ${ }^{55}$, S.L. Lloyd ${ }^{75}$, E. Lobodzinska ${ }^{41}$, P. Loch ${ }^{6}$, W.S. Lockman ${ }^{137}$, S. Lockwitz ${ }^{175}$, T. Loddenkoetter ${ }^{20}$, F.K. Loebinger ${ }^{82}$, A. Loginov ${ }^{175}$, C.W. Loh ${ }^{168}$, T. Lohse ${ }^{15}$, K. Lohwasser ${ }^{48}$, M. Lokajicek ${ }^{125}$, R.E. Long ${ }^{71}$, L. Lopes ${ }^{124 a, b}$, D. Lopez Mateos ${ }^{34, a a}$, M. Losada ${ }^{162}$, P. Loscutoff ${ }^{14}$,

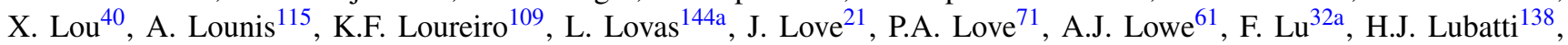
C. Luci ${ }^{132 a, 132 b}$, A. Lucotte ${ }^{55}$, A. Ludwig ${ }^{43}$, D. Ludwig ${ }^{41}$, I. Ludwig ${ }^{48}$, F. Luehring ${ }^{61}$, D. Lumb ${ }^{48}$, L. Luminari ${ }^{132 a}$, E. Lund ${ }^{117}$, B. Lund-Jensen ${ }^{147}$, B. Lundberg ${ }^{79}$, J. Lundberg ${ }^{29}$, J. Lundquist ${ }^{35}$, D. Lynn ${ }^{24}$, J. Lys ${ }^{14}$, E. Lytken ${ }^{79}$, H. Ma ${ }^{24}$, L.L. Ma ${ }^{172}$, J.A. Macana Goia ${ }^{93}$, G. Maccarrone ${ }^{47}$, A. Macchiolo ${ }^{99}$, B. Maček ${ }^{74}$, J. Machado Miguens ${ }^{124 a, b}$, R. Mackeprang $^{35}$, R.J. Madaras ${ }^{14}$, W.F. Mader ${ }^{43}$, R. Maenner ${ }^{58 c}$, T. Maeno ${ }^{24}$, P. Mättig ${ }^{174}$, S. Mättig ${ }^{41}$, P.J. Magalhaes Martins ${ }^{124 a, h}$, E. Magradze ${ }^{51}$, Y. Mahalalel ${ }^{153}$, K. Mahboubi ${ }^{48}$, A. Mahmood ${ }^{1}$, C. Maiani ${ }^{132 a, 132 b}$, C. Maidantchik ${ }^{23 a}$, A. Maio ${ }^{124 a, r}$, S. Majewski ${ }^{24}$, Y. Makida ${ }^{66}$, M. Makouski ${ }^{128}$, N. Makovec ${ }^{115}$, P. Mal ${ }^{6}$, Pa. Malecki ${ }^{38}$, P. Malecki ${ }^{38}$, V.P. Maleev ${ }^{121}$, F. Malek ${ }^{55}$, U. Mallik ${ }^{63}$, D. Malon ${ }^{5}$, S. Maltezos ${ }^{9}$, V. Malyshev ${ }^{107}$, S. Malyukov ${ }^{65}$, R. Mameghani ${ }^{98}$, J. Mamuzic ${ }^{41}$, L. Mandelli ${ }^{89 a}$, I. Mandić ${ }^{74}$, R. Mandrysch ${ }^{15}$, J. Maneira ${ }^{124 a}$, P.S. Mangeard ${ }^{88}$, I.D. Manjavidze ${ }^{65}$, A. Mann ${ }^{54}$, P.M. Manning ${ }^{137}$, A. Manousakis-Katsikakis ${ }^{8}$, B. Mansoulie ${ }^{136}$, A. Mapellii ${ }^{29}$, L. Mapelli ${ }^{29}$, L. March ${ }^{80}$, J.F. Marchand ${ }^{29}$, F. Marchese $^{133 a, 133 b}$, G. Marchiori ${ }^{78}$, M. Marcisovsky ${ }^{125,0}$, C.P. Marino ${ }^{61}$, F. Marroquim ${ }^{23 a}$, Z. Marshall ${ }^{34, a a}$, S. Marti-Garcia ${ }^{167}$, A.J. Martin ${ }^{75}$, B. $\operatorname{Martin}^{29}$, B. Martin ${ }^{88}$, F.F. Martin ${ }^{120}$, J.P. Martin ${ }^{93}$, T.A. Martin ${ }^{17}$, B. Martin dit Latour ${ }^{49}$, M. Martinez ${ }^{11}$, V. Martinez Outschoorn ${ }^{57}$, A.C. Martyniuk ${ }^{82}$, F. Marzano ${ }^{132 a}$, A. Marzin $^{136}$, L. Masetti ${ }^{81}$, T. Mashimo ${ }^{155}$, R. Mashinistov $^{96}$, J. Masik ${ }^{82}$, A.L. Maslennikov ${ }^{107}$, I. Massa ${ }^{19 a, 19 b}$, N. Massol ${ }^{4}$, A. Mastroberardino ${ }^{36 a, 36 b}$, T. Masubuchi ${ }^{155}$, P. Matri$\operatorname{con}^{115}$, H. Matsunaga ${ }^{155}$, T. Matsushita ${ }^{67}$, C. Mattravers ${ }^{118, a b}$, S.J. Maxfield ${ }^{73}$, A. Mayne ${ }^{139}$, R. Mazini ${ }^{151}$, M. Mazur ${ }^{48}$, S.P. Mc Kee ${ }^{87}$, A. McCarn ${ }^{165}$, R.L. McCarthy ${ }^{148}$, N.A. McCubbin $^{129}$, K.W. McFarlane ${ }^{56}$, H. McGlone ${ }^{53}$, G. Mchedlidze ${ }^{51}$, S.J. McMahon ${ }^{129}$, R.A. McPherson ${ }^{169, \mathrm{k}}$, A. Meade ${ }^{84}$, J. Mechnich ${ }^{105}$, M. Mechtel ${ }^{174}$, M. Medinnis ${ }^{41}$, R. Meera-Lebbai ${ }^{111}$, T. Meguro ${ }^{116}$, S. Mehlhase ${ }^{41}$, A. Mehta ${ }^{73}$, K. Meier ${ }^{58 a}$, B. Meirose ${ }^{48}$, C. Melachrinos ${ }^{30}$, B.R. Mellado Garcia ${ }^{172}$, L. Mendoza Navas ${ }^{162}$, Z. Meng ${ }^{151, a c}$, S. Menke ${ }^{99}$, E. Meoni ${ }^{11}$, P. Mermod ${ }^{118}$, L. Merola ${ }^{102 a, 102 b}$, C. Meroni ${ }^{89 a}$, F.S. Merritt ${ }^{30}$, 
A.M. Messina ${ }^{29}$, J. Metcalfe ${ }^{103}$, A.S. $M^{6 t e^{64}}$, J.-P. Meyer ${ }^{136}$, J. Meyer ${ }^{173}$, J. Meyer ${ }^{54}$, T.C. Meyer ${ }^{29}$, W.T. Meyer ${ }^{64}$, J. Miao ${ }^{32 \mathrm{~d}}$, S. Michal $^{29}$, L. Micu ${ }^{25 a}$, R.P. Middleton ${ }^{129}$, S. Migas ${ }^{73}$, L. Mijović ${ }^{74}$, G. Mikenberg ${ }^{171}$, M. Mikestikova ${ }^{125}$, M. Mikuž ${ }^{74}$, D.W. Miller ${ }^{143}$, W.J. Mills ${ }^{168}$, C. Mills $^{57}$, A. Milov ${ }^{171}$, D.A. Milstead ${ }^{146 a, 146 b}$, D. Milstein ${ }^{171}$, A.A. Minaenko ${ }^{128}$, M. Miñano ${ }^{167}$, I.A. Minashvili ${ }^{65}$, A.I. Mincer ${ }^{108}$, B. Mindur ${ }^{37}$, M. Mineev ${ }^{65}$, Y. Ming ${ }^{130}$, L.M. Mir ${ }^{11}$, G. Mirabelli ${ }^{132 a}$, S. Misawa ${ }^{24}$, A. Misiejuk ${ }^{76}$, J. Mitrevski ${ }^{137}$, V.A. Mitsou ${ }^{167}$, S. Mitsui ${ }^{160}$, P.S. Miyagawa ${ }^{82}$, K. Miyazaki ${ }^{67}$, J.U. Mjörnmark ${ }^{79}$, T. Moa ${ }^{146 a, 146 b}$, V. Moeller ${ }^{27}$, K. Mönig ${ }^{41}$, N. Möser ${ }^{20}$, W. Mohr ${ }^{48}$, S. Mohrdieck-Möck ${ }^{99}$, R. MolesValls $^{167}$, J. Molina-Perez ${ }^{29}$, J. Monk ${ }^{77}$, E. Monnier ${ }^{83}$, S. Montesano ${ }^{89 a, 89 b}$, F. Monticelli ${ }^{70}$, R.W. Moore ${ }^{2}$, C.Mora Herrera $^{49}$, A. Moraes ${ }^{53}$, A. Morais ${ }^{124 a, b}$, J. Morel ${ }^{54}$, G. Morello ${ }^{36 a, 36 b}$, D. Moreno ${ }^{81}$, M.Moreno Llácer ${ }^{167}$, P. Morettini ${ }^{50 a}$, M. Morii ${ }^{57}$, A.K. Morley ${ }^{86}$, G. Mornacchi ${ }^{29}$, J.D. Morris ${ }^{75}$, H.G. Moser ${ }^{99}$, M. Mosidze ${ }^{51}$, J. Moss ${ }^{109}$, R. Mount ${ }^{143}$, E. Mountricha $^{136}$, S.V. Mouraviev ${ }^{94}$, E.J.W. Moyse ${ }^{84}$, M. Mudrinic ${ }^{12 b}$, F. Mueller ${ }^{58 a}$, J. Mueller ${ }^{123}$, K. Mueller ${ }^{20}$, T.A. Müller ${ }^{98}$, D. Muenstermann ${ }^{42}$, A. Muir ${ }^{168}$, Y. Munwes ${ }^{153}$, W.J. Murray ${ }^{129}$, I. Mussche ${ }^{105}$, E. Musto ${ }^{102 a, 102 b}$, A.G. Myagkov ${ }^{128}$, M. Myska ${ }^{125, \mathrm{o}}$, J. Nadal ${ }^{11}$, K. Nagai ${ }^{160}$, K. Nagano ${ }^{66}$, Y. Nagasaka ${ }^{60}$, A.M. Nairz ${ }^{29}$, K. Nakamura ${ }^{155}$, I. Nakano ${ }^{110}$, G. Nanava ${ }^{20}$, A. Napier ${ }^{161}$, M. Nash ${ }^{77}$,ad , N.R. Nation ${ }^{21}$, T. Nattermann ${ }^{20}$, T. Naumann ${ }^{41}$, G. Navarro ${ }^{162}$, S.K. Nderitu ${ }^{20}$, H.A. Neal ${ }^{87}$, E. Nebot ${ }^{80}$, P. Nechaeva ${ }^{94}$, A. Negri ${ }^{119 a, 119 b}$, G. Negri ${ }^{29}$, A. Nelson ${ }^{64}$, S. Nelson ${ }^{143}$, T.K. Nelson ${ }^{143}$, S. Nemecek $^{125}$, P. Nemethy ${ }^{108}$, A.A. Nepomuceno ${ }^{23 a}$, M. Nessi ${ }^{29}$, M.S. Neubauer ${ }^{165}$, A. Neusiedl ${ }^{81}$, R.M. Neves ${ }^{108}$, P. Nevski ${ }^{24}$, R.B. Nickerson ${ }^{118}$, R. Nicolaidou ${ }^{136}$, L. Nicolas ${ }^{139}$, G. Nicoletti ${ }^{47}$, B. Nicquevert ${ }^{29}$, F. Niedercorn ${ }^{115}$, J. Nielsen ${ }^{137}$, A. Nikiforov $^{15}$, K. Nikolaev ${ }^{65}$, I. Nikolic-Audit ${ }^{78}$, K. Nikolopoulos ${ }^{8}$, H. Nilsen ${ }^{48}$, P. Nilsson ${ }^{7}$, A. Nisati ${ }^{132 a}$, T. Nishiyama ${ }^{67}$, R. Nisius ${ }^{99}$, L. Nodulman ${ }^{5}$, M. Nomachi ${ }^{116}$, I. Nomidis ${ }^{154}$, M. Nordberg ${ }^{29}$, B. Nordkvist ${ }^{146 a, 146 b}$, D. Notz ${ }^{41}$, J. Novakova $^{126}$, M. Nozaki ${ }^{66}$, M. Nožička ${ }^{41}$, I.M. Nugent ${ }^{159 a}$, A.-E. Nuncio-Quiroz ${ }^{20}$, G. Nunes Hanninger ${ }^{20}$, T. Nunnemann $^{98}$, E. Nurse ${ }^{77}$, D.C. O’Neil ${ }^{142}$, V. O’Shea ${ }^{53}$, F.G. Oakham ${ }^{28, g}$, H. Oberlack ${ }^{99}$, A. Ochi ${ }^{67}$, S. Oda ${ }^{155}$, S. Odaka ${ }^{66}$, J. Odier ${ }^{83}$, H. Ogren ${ }^{61}$, A. Oh $^{82}$, S.H. Oh $^{44}$, C.C. Ohm ${ }^{146 a, 146 b}$, T. Ohshima ${ }^{101}$, T. Ohsugi ${ }^{59}$, S. Okada ${ }^{67}$, H. Okawa ${ }^{163}$, Y. Okumura ${ }^{101}$, T. Okuyama ${ }^{155}$, A.G. Olchevski ${ }^{65}$, M. Oliveira ${ }^{124 a, h}$, D. Oliveira Damazio ${ }^{24}$, E. Oliver Garcia ${ }^{167}$, D. Olivito $^{120}$, A. Olszewski ${ }^{38}$, J. Olszowska ${ }^{38}$, C. Omachi ${ }^{67, a e}$, A. Onofre ${ }^{124 a, a f}$, P.U.E. Onyisi ${ }^{30}$, C.J. Oram ${ }^{159 a}$, M.J. Oreglia ${ }^{30}$, Y. Oren ${ }^{153}$, D. Orestano ${ }^{134 a, 134 b}$, I. Orlov ${ }^{107}$, C. Oropeza Barrera ${ }^{53}$, R.S. Orr ${ }^{158}$, E.O. Ortega ${ }^{130}$, B. Osculati ${ }^{50 a, 50 b}$, R. Ospanov ${ }^{120}$, C. Osuna ${ }^{11}$, G. Otero y Garzon ${ }^{26}$, J.P Ottersbach ${ }^{105}$, F. Ould-Saada ${ }^{117}$, A. Ouraou ${ }^{136}$, Q. Ouyang ${ }^{32 a}$, M. Owen ${ }^{82}$, S. Owen ${ }^{139}$, A. Oyarzun ${ }^{31 b}$, V.E. Ozcan ${ }^{77}$, N. Ozturk ${ }^{7}$, A. Pacheco Pages ${ }^{11}$, C. Padilla Aranda ${ }^{11}$, E. Paganis $^{139}$, F. Paige ${ }^{24}$, K. Pajchel ${ }^{117}$, S. Palestini2 ${ }^{29}$, D. Pallin ${ }^{33}$, A. Palma ${ }^{124 a, b}$, J.D. Palmer ${ }^{17}$, Y.B. Pan ${ }^{172}$, E. Panagiotopoulou $^{9}$, B. Panes ${ }^{31 a}$, N. Panikashvili ${ }^{87}$, S. Panitkin ${ }^{24}$, D. Pantea ${ }^{25 a}$, M. Panuskova ${ }^{125}$, V. Paolone ${ }^{123}$, Th.D. Papadopoulou $^{9}$, S.J. Park ${ }^{54}$, W. Park ${ }^{24, a g}$, M.A. Parker ${ }^{27}$, F. Parodi ${ }^{50,50 b}$, J.A. Parsons ${ }^{34}$, U. Parzefall ${ }^{48}$, E. Pasqualucci ${ }^{132 a}$, A. Passeri ${ }^{134 a}$, F. Pastore ${ }^{134 a, 134 b}$, Fr. Pastore ${ }^{29}$, G. Pásztor ${ }^{49, a h}$, S. Pataraia ${ }^{99}$, N. Patel $^{150}$, J.R. Pater ${ }^{82}$, S. Patricelli $^{102 a, 102 b}$, T. Pauly ${ }^{29}$, M. Pecsy ${ }^{144 a}$, M.I. Pedraza Morales ${ }^{172}$, S.V. Peleganchuk ${ }^{107}$, H. Peng ${ }^{172}$, A. Penson ${ }^{34}$, J. Penwell ${ }^{61}$, M. Perantoni $^{23 a}$, K. Perez ${ }^{34, a a}$, E. Perez Codina ${ }^{11}$, M.T. Pérez García-Estañ ${ }^{167}$, V. Perez Reale ${ }^{34}$, L. Perini ${ }^{89 a, 89 b}$, H. Pernegger ${ }^{29}$, R. Perrino $^{72 a}$, S. Persembe ${ }^{3 a}$, P. Perus ${ }^{115}$, V.D. Peshekhonov ${ }^{65}$, B.A. Petersen ${ }^{29}$, T.C. Petersen ${ }^{35}$, E. Petit ${ }^{83}$, C. Petridou ${ }^{154}$, E. Petrolo ${ }^{132 a}$, F. Petrucci ${ }^{134 a, 134 b}$, D. Petschull ${ }^{41}$, M. Petteni ${ }^{142}$, R. Pezoa ${ }^{31 b}$, B. Pfeifer ${ }^{48}$, A. Phan ${ }^{86}$, A.W. Phillips ${ }^{27}$, G. Piacquadio ${ }^{29}$, E. Piccaro ${ }^{75}$, M. Piccinini ${ }^{19 a, 19 b}$, R. Piegaia ${ }^{26}$, J.E. Pilcher ${ }^{30}$, A.D. Pilkington ${ }^{82}$, J. Pina ${ }^{124 a, r}$, M. Pinamonti ${ }^{164 a, 164 c}$, J.L. Pinfold ${ }^{2}$, B. Pinto ${ }^{124 a, b}$, C. Pizio ${ }^{89 a, 89 b}$, R. Placakyte ${ }^{41}$, M. Plamondon ${ }^{169}$, M.-A. Pleier ${ }^{24}$, A. Poblaguev ${ }^{175}$, S. Poddar ${ }^{58 a}$, F. Podlyski ${ }^{33}$, L. Poggioli ${ }^{115}$, M. Pohl ${ }^{49}$, F. Polci ${ }^{55}$, G. Polesello ${ }^{119 a}$, A. Policicchio ${ }^{138}$, A. Polini ${ }^{19 a}$, J. Poll ${ }^{75}$, V. Polychronakos ${ }^{24}$, D. Pomeroy ${ }^{22}$, K. Pommès $^{29}$, L. Pontecorvo ${ }^{132 a}$, B.G. Pope ${ }^{88}$, G.A. Popeneciu ${ }^{25 a}$, D.S. Popovic ${ }^{12 a}$, A. Poppleton ${ }^{29}$, X. Portell Bueso ${ }^{48}$, R. Porter ${ }^{163}$, G.E. Pospelov ${ }^{99}$, S. Pospisil ${ }^{127}$, M. Potekhin ${ }^{24}$, I.N. Potrap $^{99}$, C.J. Potter ${ }^{149}$, C.T. Potter ${ }^{85}$, K.P. Potter ${ }^{82}$, G. Poulard ${ }^{29}$, J. Poveda ${ }^{172}$, R. Prabhu ${ }^{20}$, P. Pralavorio ${ }^{83}$, S. Prasad ${ }^{57}$, R. Pravahan $^{7}$, L. Pribyl $^{29}$, D. Price ${ }^{61}$, L.E. Price ${ }^{5}$, P.M. Prichard ${ }^{73}$, D. Prieur ${ }^{123}$, M. Primavera ${ }^{72 a}$, K. Prokofiev ${ }^{29}$, F. Prokoshin ${ }^{31 b}$, S. Protopopescu ${ }^{24}$, J. Proudfoot ${ }^{5}$, X. Prudent ${ }^{43}$, H. Przysiezniak ${ }^{4}$, S. Psoroulas ${ }^{20}$, E. Ptacek ${ }^{114}$, J. Purdham $^{87}$, M. Purohit ${ }^{24, a i}$, P. Puzo ${ }^{115}$, Y. Pylypchenko ${ }^{117}$, J. Qian ${ }^{87}$, W. Qian ${ }^{129}$, Z. Qin ${ }^{41}$, A. Quadt ${ }^{54}$, D.R. Quarrie ${ }^{14}$, W.B. Quayle ${ }^{172}$, F. Quinonez ${ }^{31 a}$, M. Raas ${ }^{104}$, V. Radeka ${ }^{24}$, V. Radescu ${ }^{58 b}$, B. Radics ${ }^{20}$, T. Rador ${ }^{18 a}$, F. Ragusa ${ }^{89 a, 89 b}$, G. Rahal $^{180}$, A.M. Rahimi ${ }^{109}$, S. Rajagopalan ${ }^{24}$, M. Rammensee ${ }^{48}$, M. Rammes ${ }^{141}$, F. Rauscher ${ }^{98}$, E. Rauter ${ }^{99}$, M. Raymond $^{29}$, A.L. Read ${ }^{117}$, D.M. Rebuzzi ${ }^{119 a, 119 b}$, A. Redelbach ${ }^{173}$, G. Redlinger ${ }^{24}$, R. Reece ${ }^{120}$, K. Reeves ${ }^{40}$, E. ReinherzAronis $^{153}$, A. Reinsch ${ }^{114}$, I. Reisinger ${ }^{42}$, D. Reljic ${ }^{12 a}$, C. Rembser ${ }^{29}$, Z.L. Ren ${ }^{151}$, P. Renkel ${ }^{39}$, S. Rescia ${ }^{24}$, M. Rescigno $^{132 a}$, S. Resconi ${ }^{89 a}$, B. Resende ${ }^{136}$, P. Reznicek ${ }^{126}$, R. Rezvani ${ }^{158}$, A. Richards ${ }^{77}$, R. Richter ${ }^{99}$, E. Richter-Was ${ }^{38, \text { aj }}$, M. Ridel ${ }^{78}$, M. Rijpstra ${ }^{105}$, M. Rijssenbeek ${ }^{148}$, A. Rimoldi ${ }^{119 a, 119 b}$, L. Rinaldi ${ }^{19 a}$, R.R. Rios ${ }^{39}$, I. Riu ${ }^{11}$, F. Rizatdinova ${ }^{112}$, E. Rizvi ${ }^{75}$, D.A. Roa Romero ${ }^{162}$, S.H. Robertson ${ }^{85, \mathrm{k}}$, A. Robichaud-Veronneau ${ }^{49}$, D. Robinson ${ }^{27}$, J.E.M. Robinson ${ }^{77}$, M. Robinson ${ }^{114}$, A. Robson ${ }^{53}$, J.G. Rocha de Lima ${ }^{106}$, C. Roda ${ }^{122 a, 122 b}$, D. Roda Dos Santos ${ }^{29}$, D. Rodriguez ${ }^{162}$, Y. Rodriguez Garcia ${ }^{15}$, S. Roe ${ }^{29}$, O. Røhne ${ }^{117}$, V. Rojo ${ }^{1}$, S. Rolli ${ }^{161}$, A. Romaniouk ${ }^{96}$, V.M. Romanov ${ }^{65}$, G. Romeo ${ }^{26}$, D. Romero Maltrana ${ }^{31 a}$, L. Roos ${ }^{78}$, E. $\operatorname{Ros}^{167}$, S. Rosati ${ }^{138}$, G.A. Rosenbaum ${ }^{158}$, L. Rosselet ${ }^{49}$, V. Rossetti ${ }^{11}$, L.P. Rossi ${ }^{50 a}$, M. Rotaru ${ }^{25 a}$, J. Rothberg $^{138}$, D. Rousseau ${ }^{115}$, C.R. Royon ${ }^{136}$, A. Rozanov ${ }^{83}$, Y. Rozen ${ }^{152}$, X. Ruan ${ }^{115}$, B. Ruckert ${ }^{98}$, N. Ruckstuh1 ${ }^{105}$, V.I. Rud ${ }^{97}$, 
G. Rudolph ${ }^{62}$, F. Rühr ${ }^{58 a}$, F. Ruggieri ${ }^{134 a}$, A. Ruiz-Martinez ${ }^{64}$, L. Rumyantsev ${ }^{65}$, Z. Rurikova ${ }^{48}$, N.A. Rusakovich ${ }^{65}$, J.P. Rutherfoord ${ }^{6}$, C. Ruwiedel ${ }^{20}$, P. Ruzicka ${ }^{125,0}$, Y.F. Ryabov ${ }^{121}$, P. Ryan ${ }^{88}$, G. Rybkin ${ }^{115}$, S. Rzaeva ${ }^{10}$, A.F. Saavedra $^{150}$, H.F-W. Sadrozinski ${ }^{137}$, R. Sadykov ${ }^{65}$, F. Safai Tehrani ${ }^{132 a, 132 b}$, H. Sakamoto ${ }^{155}$, G. Salamanna ${ }^{105}$, A. Salamon ${ }^{133 a}$, M. Saleem ${ }^{111}$, D. Salihagic ${ }^{99}$, A. Salnikov ${ }^{143}$, J. Salt ${ }^{167}$, B.M. Salvachua Ferrando ${ }^{5}$, D. Salvatore ${ }^{36 a, 36 b}$, F. Salvatore ${ }^{149}$, A. Salvucci ${ }^{47}$, A. Salzburger ${ }^{29}$, D. Sampsonidis ${ }^{154}$, B.H. Samset ${ }^{117}$, H. Sandaker ${ }^{13}$, H.G. Sander ${ }^{81}$, M.P. Sanders ${ }^{98}$, M. Sandhoff $^{174}$, P. Sandhu ${ }^{158}$, R. Sandstroem ${ }^{105}$, S. Sandvoss ${ }^{174}$, D.P.C. Sankey ${ }^{129}$, A. Sansoni ${ }^{47}$, C. Santamarina Rios ${ }^{85}$, C. Santoni $^{33}$, R. Santonico ${ }^{133 a, 133 b}$, J.G. Saraiva ${ }^{124 a, r}$, T. Sarangi ${ }^{172}$, E. Sarkisyan-Grinbaum ${ }^{7}$, F. Sarri ${ }^{122 a, 122 b}$, O. Sasaki ${ }^{66}$, N. Sasao ${ }^{68}$, I. Satsounkevitch ${ }^{90}$, G. Sauvage ${ }^{4}$, P. Savard ${ }^{158, g}$, A.Y. Savine ${ }^{6}$, V. Savinov ${ }^{123}$, L. Sawyer ${ }^{24, a k}$, D.H. Saxon ${ }^{53}$, L.P. Says ${ }^{33}$, C. Sbarra $^{19 a, 19 b}$, A. Sbrizzi19a,19b ${ }^{19}$ D.A. Scannicchio ${ }^{29}$, J. Schaarschmidt ${ }^{43}$, P. Schacht ${ }^{99}$, U. Schäfer ${ }^{81}$, S. Schaetzel ${ }^{58 b}$, A.C. Schaffer ${ }^{115}$, D. Schaile ${ }^{98}$, R.D. Schamberger ${ }^{148}$, A.G. Schamov ${ }^{107}$, V. Scharf ${ }^{58 a}$, V.A. Schegelsky $^{121}$, D. Scheirich ${ }^{87}$, M. Schernau ${ }^{163}$, M.I. Scherzer ${ }^{14}$, C. Schiavi ${ }^{50,50 b}$, J. Schieck ${ }^{99}$, M. Schioppa ${ }^{36 a, 36 b}$, S. Schlenker ${ }^{29}$, E. Schmidt ${ }^{48}$, K. Schmieden ${ }^{20}$, C. Schmitt ${ }^{81}$, M. Schmitz ${ }^{20}$, A. Schöning ${ }^{58 b}$, M. Schott ${ }^{29}$, D. Schouten ${ }^{142}$, J. Schovan$\operatorname{cova}^{125}$, M. Schram ${ }^{85}$, A. Schreiner ${ }^{63}$, C. Schroeder ${ }^{81}$, N. Schroer ${ }^{58 c}$, M. Schroers ${ }^{174}$, J. Schultes ${ }^{174}$, H.-C. SchultzCoulon $^{58 a}$, J.W. Schumacher ${ }^{43}$, M. Schumacher ${ }^{48}$, B.A. Schumm ${ }^{137}$, Ph. Schune ${ }^{136}$, C. Schwanenberger ${ }^{82}$, A. Schwartz$\operatorname{man}^{143}$, Ph. Schwemling ${ }^{78}$, R. Schwienhorst ${ }^{88}$, R. Schwierz ${ }^{43}$, J. Schwindling ${ }^{136}$, W.G. Scott ${ }^{129}$, J. Searcy ${ }^{114}$, E. Sedykh ${ }^{121}$, E. Segura $^{11}$, S.C. Seidel ${ }^{103}$, A. Seiden ${ }^{137}$, F. Seifert ${ }^{43}$, J.M. Seixas ${ }^{23 a}$, G. Sekhniaidze ${ }^{102 a}$, D.M. Seliverstov ${ }^{121}$, B. Sell$\operatorname{den}^{146 a}$, N. Semprini-Cesari ${ }^{19 a, 19 b}$, C. Serfon ${ }^{98}$, L. Serin ${ }^{115}$, R. Seuster ${ }^{99}$, H. Severini ${ }^{111}$, M.E. Sevior ${ }^{86}$, A. Sfyrla ${ }^{29}$, E. Shabalina $^{54}$, M. Shamim 114 , L.Y. Shan ${ }^{32 a}$, J.T. Shank ${ }^{21}$, Q.T. Shao ${ }^{86}$, M. Shapiro ${ }^{14}$, P.B. Shatalov ${ }^{95}$, K. Shaw ${ }^{139}$, D. Sher$\operatorname{man}^{29}$, P. Sherwood ${ }^{77}$, A. Shibata ${ }^{108}$, M. Shimojima ${ }^{100}$, T. Shin ${ }^{56}$, A. Shmeleva ${ }^{94}$, M.J. Shochet ${ }^{30}$, M.A. Shupe ${ }^{6}$, P. Sicho $^{125}$, A. Sidoti ${ }^{15}$, F. Siegert ${ }^{77}$, J. Siegrist ${ }^{14}$, Dj. Sijacki ${ }^{12 a}$, O. Silbert ${ }^{171}$, Y. Silver ${ }^{153}$, D. Silverstein ${ }^{143}$, S.B. Silverstein $^{146 a}$, V. Simak ${ }^{127}$, Lj. Simic ${ }^{12 a}$, S. Simion ${ }^{115}$, B. Simmons ${ }^{77}$, M. Simonyan ${ }^{35}$, P. Sinervo ${ }^{158}$, N.B. Sinev ${ }^{114}$, V. Sipica ${ }^{141}$, G. Siragusa ${ }^{81}$, A.N. Sisakyan ${ }^{65}$, S.Yu. Sivoklokov ${ }^{97}$, J. Sjölin ${ }^{146 a, 146 b}$, T.B. Sjursen ${ }^{13}$, K. Skovpen ${ }^{107}$, P. Skubic ${ }^{111}$, M. Slater ${ }^{17}$, T. Slavicek ${ }^{127}$, K. Sliwa ${ }^{161}$, J. Sloper ${ }^{29}$, V. Smakhtin ${ }^{171}$, S.Yu. Smirnov ${ }^{96}$, Y. Smirnov ${ }^{24}$, L.N. Smirnova ${ }^{97}$, O. Smirnova ${ }^{79}$, B.C. Smith ${ }^{57}$, D. Smith ${ }^{143}$, K.M. Smith ${ }^{53}$, M. Smizanska ${ }^{71}$, K. Smolek ${ }^{127}$, A.A. Snesarev ${ }^{94}$, S.W. Snow ${ }^{82}$, J. Snow ${ }^{111}$, J. Snuverink ${ }^{105}$, S. Snyder ${ }^{24}$, M. Soares ${ }^{124 a}$, R. Sobie ${ }^{169, k}$, J. Sodomka ${ }^{127}$, A. Soffer ${ }^{153}$, C.A. Solans ${ }^{167}$, M. So$\operatorname{lar}^{127}$, J. Solc ${ }^{127}$, E. Solfaroli Camillocci ${ }^{132 a, 132 b}$, A.A. Solodkov ${ }^{128}$, O.V. Solovyanov ${ }^{128}$, J. Sondericker ${ }^{24}$, V. Sopko ${ }^{127}$, B. Sopko ${ }^{127}$, M. Sosebee ${ }^{7}$, A. Soukharev ${ }^{107}$, S. Spagnolo ${ }^{72 a, 72 b}$, F. Spanò ${ }^{34}$, R. Spighi ${ }^{19 a}$, G. Spigo ${ }^{29}$, F. Spila ${ }^{132 a, 132 b}$, R. Spiwoks ${ }^{29}$, M. Spousta ${ }^{126}$, B. Spurlock ${ }^{7}$, R.D. St. Denis ${ }^{53}$, T. Stahl ${ }^{141}$, J. Stahlman ${ }^{120}$, R. Stamen ${ }^{58 a}$, E. Stanecka ${ }^{29}$, R.W. Stanek ${ }^{5}$, C. Stanescu ${ }^{134 a}$, S. Stapnes ${ }^{117}$, E.A. Starchenko ${ }^{128}$, J. Stark ${ }^{55}$, P. Staroba ${ }^{125}$, P. Starovoitov ${ }^{91}$, P. Stavina ${ }^{144 a}$, G. Steele ${ }^{53}$, P. Steinbach ${ }^{43}$, P. Steinberg ${ }^{24}$, I. Stekl ${ }^{127}$, B. Stelzer ${ }^{142}$, H.J. Stelzer ${ }^{41}$, O. Stelzer-Chilton ${ }^{159 a}$, H. Stenzel ${ }^{52}$, K. Stevenson ${ }^{75}$, G.A. Stewart ${ }^{53}$, M.C. Stockton ${ }^{29}$, K. Stoerig ${ }^{48}$, G. Stoicea ${ }^{25 a}$, S. Stonjek ${ }^{99}$, P. Strachota ${ }^{126}$, A.R. Stradling ${ }^{7}$, A. Straessner ${ }^{43}$, J. Strandberg ${ }^{87}$, S. Strandberg ${ }^{14}$, A. Strandlie ${ }^{117}$, M. Strang ${ }^{109}$, M. Strauss ${ }^{111}$, P. Strizenec ${ }^{144 b}$, R. Ströhmer $^{173}$, D.M. Strom ${ }^{114}$, R. Stroynowski ${ }^{39}$, J. Strube ${ }^{129}$, B. Stugu ${ }^{13}$, P. Sturm ${ }^{174}$, D.A. Soh ${ }^{151, a l}$, D. Su ${ }^{143}$, Y. Sugaya ${ }^{116}$, T. Sugimoto ${ }^{101}$, C. Suhr ${ }^{106}$, K. Suita ${ }^{67}$, M. Suk ${ }^{126}$, V.V. Sulin ${ }^{94}$, S. Sultansoy ${ }^{3 d}$, T. Sumida ${ }^{29}$, X. Sun ${ }^{32 d}$, J.E. Sundermann ${ }^{48}$, K. Suruliz ${ }^{164 a, 164 b}$, S. Sushkov ${ }^{11}$, G. Susinno ${ }^{36 a, 36 b}$, M.R. Sutton ${ }^{139}$, Y. Suzuki ${ }^{66}$, I. Sykora ${ }^{144 a}$, T. Sykora ${ }^{126}$, T. Szymocha $^{38}$, J. Sánchez ${ }^{167}$, D. Ta ${ }^{20}$, K. Tackmann ${ }^{29}$, A. Taffard ${ }^{163}$, R. Tafirout ${ }^{159 a}$, A. Taga ${ }^{117}$, Y. Takahashi ${ }^{101}$, H. Takai $^{24}$, R. Takashima ${ }^{69}$, H. Takeda ${ }^{67}$, T. Takeshita ${ }^{140}$, M. Talby ${ }^{83}$, A. Talyshev ${ }^{107}$, M.C. Tamsett ${ }^{76}$, J. Tanaka ${ }^{155}$, R. Tanaka ${ }^{115}$, S. Tanaka ${ }^{131}$, S. Tanaka ${ }^{66}$, K. Tani ${ }^{67}$, S. Tapprogge ${ }^{81}$, D. Tardif ${ }^{158}$, S. Tarem ${ }^{152}$, F. Tarrade $^{24}$, G.F. Tartarelli ${ }^{89 a}$, P. Tas ${ }^{126}$, M. Tasevsky ${ }^{125}$, E. Tassi ${ }^{36 a, 36 b}$, M. Tatarkhanov ${ }^{14}$, C. Taylor $^{77}$, F.E. Taylor ${ }^{92}$, G.N. Taylor ${ }^{86}$, W. Taylor ${ }^{159 b}$, M.Teixeira Dias Castanheira $^{75}$, P. Teixeira-Dias ${ }^{76}$, H. Ten Kate ${ }^{29}$, P.K. Teng ${ }^{151}$, Y.D. Tennenbaum-Katan ${ }^{152}$, S. Terada ${ }^{66}$, K. Terashi $^{155}$, J. Terron ${ }^{80}$, M. Terwort ${ }^{41, w}$, M. Testa ${ }^{47}$, R.J. Teuscher ${ }^{158, k}$, J. Therhaag ${ }^{20}$, M. Thioye ${ }^{175}$, S. Thoma ${ }^{48}$, J.P. Thomas ${ }^{17}$, E.N. Thompson ${ }^{84}$, P.D. Thompson ${ }^{17}$, P.D. Thompson ${ }^{158}$, R.J. Thompson ${ }^{82}$, A.S. Thompson ${ }^{53}$, E. Thomson ${ }^{120}$, R.P. Thun ${ }^{87}$, T. Tic ${ }^{125}$, V.O. Tikhomirov ${ }^{94}$, Y.A. Tikhonov ${ }^{107}$, P. Tipton ${ }^{175}$, F.J. Tique Aires Viegas ${ }^{29}$, S. Tisserant ${ }^{83}$, B. Toczek ${ }^{37}$, T. Todorov ${ }^{4}$, S. Todorova-Nova ${ }^{161}$, B. Toggerson ${ }^{163}$, J. Tojo ${ }^{66}$, S. Tokár ${ }^{144 a}$, K. Tokunaga ${ }^{67}, \mathrm{~K}_{\text {. Tokushuku }}{ }^{66}$, K. Tollefson $^{88}$, M. Tomoto ${ }^{101}$, L. Tompkins ${ }^{14}$, K. Toms ${ }^{103}$, A. Tonoyan $^{13}$, C. Topfel ${ }^{16}$, N.D. Topilin ${ }^{65}$, I. Torchiani ${ }^{29}$, E. Torrence ${ }^{114}$, E. Torró Pastor ${ }^{167}$, J. Toth ${ }^{83, \text { ah }, \text { F. Touchard }}{ }^{83}$, D.R. Tovey ${ }^{139}$, T. Trefzger ${ }^{173}$, L. Tremblet ${ }^{29}$, A. Tricoli ${ }^{29}$, I.M. Trigger ${ }^{159 a}$,

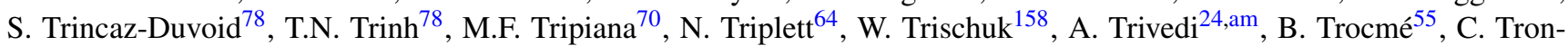
$\operatorname{con}^{89 a}$, A. Trzupek ${ }^{38}$, C. Tsarouchas ${ }^{9}$, J.C-L. Tseng ${ }^{118}$, M. Tsiakiris ${ }^{105}$, P.V. Tsiareshka ${ }^{90}$, D. Tsionou ${ }^{139}$, G. Tsipolitis $^{9}$, V. Tsiskaridze ${ }^{51}$, E.G. Tskhadadze ${ }^{51}$, I.I. Tsukerman ${ }^{95}$, V. Tsulaia ${ }^{123}$, J.-W. Tsung ${ }^{20}$, S. Tsuno ${ }^{66}$, D. Tsybychev ${ }^{148}$, J.M. Tuggle ${ }^{30}$, D. Turecek ${ }^{127}$, I. Turk Cakir ${ }^{3 e}$, E. Turlay ${ }^{105}$, P.M. Tuts ${ }^{34}$, M.S. Twomey ${ }^{138}$, M. Tylmad ${ }^{146 a, 146 b}$, M. Tyndel ${ }^{129}$, K. Uchida ${ }^{116}$, I. Ueda ${ }^{155}$, R. Ueno ${ }^{28}$, M. Ugland ${ }^{13}$, M. Uhlenbrock ${ }^{20}$, M. Uhrmacher ${ }^{54}$, F. Ukegawa ${ }^{160}$, G. Unal ${ }^{29}$, A. Undrus $^{24}$, G. Unel ${ }^{163}$, Y. Unno ${ }^{66}$, D. Urbaniec ${ }^{34}$, E. Urkovsky ${ }^{153}$, P. Urquijo ${ }^{49}$ an, P. Urrejola ${ }^{31 a}$, G. Usai ${ }^{7}$, M. Uslenghi ${ }^{119 a, 119 b}$, L. Vacavant ${ }^{83}$, V. Vacek ${ }^{127}$, B. Vachon ${ }^{85}$, S. Vahsen ${ }^{14}$, P. Valente ${ }^{132 a}$, S. Valentinetti ${ }^{19 a, 19 b}$, S. Valkar ${ }^{126}$, E. Valladolid Gallego ${ }^{167}$, S. Vallecorsa ${ }^{152}$, J.A. Valls Ferrer ${ }^{167}$, H. van der Graaf ${ }^{105}$, E. van der Kraaij ${ }^{105}$, E. van der Poel ${ }^{105}$, D. van der $\operatorname{Ster}^{29}$, 
N. van Eldik $^{84}$, P. van Gemmeren ${ }^{5}$, Z. van Kesteren ${ }^{105}$, I. van Vulpen ${ }^{105}$, W. Vandelli29 ${ }^{29}$ A. Vaniachine ${ }^{5}$, P. Vankov ${ }^{73}$, F. Vannucci $^{78}$, R. Vari ${ }^{132 a}$, E.W. Varnes ${ }^{6}$, D. Varouchas ${ }^{14}$, A. Vartapetian ${ }^{7}$, K.E. Varvell ${ }^{150}$, V.I. Vassilakopoulos ${ }^{56}$, F. Vazeille ${ }^{33}$,

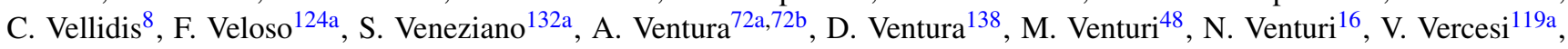
M. Verducci ${ }^{138}$, W. Verkerke ${ }^{105}$, J.C. Vermeulen ${ }^{105}$, M.C. Vetterli ${ }^{142, \mathrm{~g}}$, I. Vichou ${ }^{165}$, T. Vickey ${ }^{118}$, G.H.A. Viehhauser ${ }^{118}$, M. Villa ${ }^{19 a}$,19b, E.G. Villani ${ }^{129}$, M. Villaplana Perez ${ }^{167}$, E. Vilucchi ${ }^{47}$, M.G. Vincter ${ }^{28}$, E. Vinek ${ }^{29}$, V.B. Vinogradov ${ }^{65}$, S. Viret ${ }^{33}$, J. Virzi ${ }^{14}$, A. Vitale ${ }^{19 a, 19 b}$, O. Vitells ${ }^{171}$, I. Vivarelli ${ }^{48}$, F. Vives Vaque ${ }^{11}$, S. Vlachos ${ }^{9}$, M. Vlasak ${ }^{127}$, N. Vlasov ${ }^{20}$, A. Vogel ${ }^{20}$, P. Vokac ${ }^{127}$, M. Volpi ${ }^{11}$, H. von der Schmitt ${ }^{99}$, J. von Loeben ${ }^{99}$, H. von Radziewski ${ }^{48}$, E. von Toerne ${ }^{20}$, V. Vorobel $^{126}$, V. Vorwerk ${ }^{11}$, M. $\operatorname{Vos}^{167}$, R. Voss $^{29}$, T.T. Voss ${ }^{174}$, J.H. Vossebeld ${ }^{73}$, N. Vranjes ${ }^{12 a}$, M. Vranjes Milosavljevic ${ }^{12 a}$, V. Vrba ${ }^{125}$, M. Vreeswijk ${ }^{105}$, T. Vu Anh ${ }^{81}$, D. Vudragovic ${ }^{12 a}$, R. Vuillermet ${ }^{29}$, I. Vukotic ${ }^{115}$, P. Wagner ${ }^{120}$, J. Walbersloh ${ }^{42}$, J. Walder ${ }^{71}$, R. Walker ${ }^{98}$, W. Walkowiak ${ }^{141}$, R. Wall ${ }^{175}$, C. Wang ${ }^{44}$, H. Wang ${ }^{172}$, J. Wang ${ }^{55}$, S.M. Wang ${ }^{151}$, A. Warburton $^{85}$, C.P. Ward ${ }^{27}$, M. Warsinsky ${ }^{48}$, R. Wastie ${ }^{118}$, P.M. Watkins ${ }^{17}$, A.T. Watson ${ }^{17}$, M.F. Watson ${ }^{17}$, G. Watts ${ }^{138}$, S. Watts ${ }^{82}$, A.T. Waugh ${ }^{150}$, B.M. Waugh ${ }^{77}$, M.D. Weber ${ }^{16}$, M. Weber ${ }^{129}$, M.S. Weber ${ }^{16}$, P. Weber ${ }^{54}$, A.R. Weidberg ${ }^{118}$, J. Weingarten $^{54}$, C. Weiser ${ }^{48}$, H. Wellenstein ${ }^{22}$, P.S. Wells ${ }^{29}$, T. Wenaus ${ }^{24}$, S. Wendler ${ }^{123}$, Z. Weng ${ }^{151, \text { ao }}$, T. Wengler ${ }^{82}$, S. Wenig ${ }^{29}$, N. Wermes ${ }^{20}$, M. Werner ${ }^{48}$, P. Werner ${ }^{29}$, M. Werth ${ }^{163}$, U. Werthenbach ${ }^{141}$, M. Wessels ${ }^{58 a}$, K. Whalen ${ }^{28}$, A. White ${ }^{7}$, M.J. White ${ }^{27}$, S. White ${ }^{24}$, S.R. Whitehead ${ }^{118}$, D. Whiteson ${ }^{163}$, D. Whittington ${ }^{61}$, F. Wicek ${ }^{115}$, D. Wicke ${ }^{81}$, F.J. Wickens ${ }^{129}$, W. Wiedenmann ${ }^{172}$, M. Wielers ${ }^{129}$, P. Wienemann ${ }^{20}$, C. Wiglesworth ${ }^{73}$, L.A.M. Wiik ${ }^{48}$, A. Wildauer ${ }^{167}$, M.A. Wildt ${ }^{41, w}$, H.G. Wilkens ${ }^{29}$, E. Williams ${ }^{34}$, H.H. Williams ${ }^{120}$, S. Willocq ${ }^{84}$, J.A. Wilson ${ }^{17}$, M.G. Wilson ${ }^{143}$, A. Wilson ${ }^{87}$, I. WingerterSeez $^{4}$, F. Winklmeier ${ }^{29}$, M. Wittgen ${ }^{143}$, M.W. Wolter ${ }^{38}$, H. Wolters ${ }^{124 a, h}$, B.K. Wosiek ${ }^{38}$, J. Wotschack ${ }^{29}$, M.J. Woudstra ${ }^{84}$, K. Wraight ${ }^{53}$, C. Wright ${ }^{53}$, D. Wright ${ }^{143}$, B. Wrona ${ }^{73}$, S.L. Wu ${ }^{172}$, X. Wu ${ }^{49}$, E. Wulf ${ }^{34}$, B.M. Wynne ${ }^{45}$, L. Xaplanteris ${ }^{9}$, S. Xella ${ }^{35}$, S. Xie ${ }^{48}$, D. Xu ${ }^{139}$, M. Yamada ${ }^{160}$, A. Yamamoto ${ }^{66}$, K. Yamamoto ${ }^{64}$, S. Yamamoto ${ }^{155}$, T. Yamamura ${ }^{155}$, J. Yamaoka $^{44}$, T. Yamazaki ${ }^{155}$, Y. Yamazaki ${ }^{67}$, Z. Yan ${ }^{21}$, H. Yang ${ }^{87}$, U.K. Yang ${ }^{82}$, Z. Yang ${ }^{146 a, 146 b}$, W.-M. Yao ${ }^{14}$, Y. Yao ${ }^{14}$,

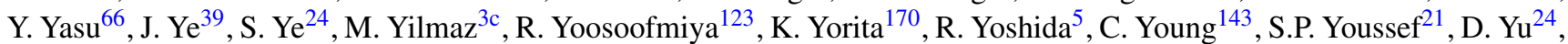
J. Yu $^{7}$, L. Yuan ${ }^{78}$, A. Yurkewicz ${ }^{148}$, R. Zaidan ${ }^{63}$, A.M. Zaitsev ${ }^{128}$, Z. Zajacova ${ }^{29}$, V. Zambrano ${ }^{47}$, L. Zanello ${ }^{132 a, 132 b}$, A. Zaytsev $^{107}$, C. Zeitnitz ${ }^{174}$, M. Zeller ${ }^{175}$, A. Zemla ${ }^{38}$, C. Zendler ${ }^{20}$, O. Zenin ${ }^{128}$, T. Ženišr ${ }^{144 a}$, Z. Zenonos ${ }^{122 a, 122 b}$, S. Zenz ${ }^{14}$, D. Zerwas ${ }^{115}$, G. Zevi della Porta ${ }^{57}$, Z. Zhan ${ }^{32 d}$, H. Zhang ${ }^{83}$, J. Zhang ${ }^{5}$, Q. Zhang ${ }^{5}$, X. Zhang ${ }^{32 d}$, L. Zhao ${ }^{108}$, T. Zhao ${ }^{138}$, Z. Zhao ${ }^{32 b}$, A. Zhemchugov ${ }^{65}$, J. Zhong ${ }^{151, a p}$, B. Zhou ${ }^{87}$, N. Zhou ${ }^{34}$, Y. Zhou ${ }^{151}$, C.G. Zhu ${ }^{32 d}$, H. Zhu ${ }^{41}$, Y. Zhu ${ }^{172}$, X. Zhuang ${ }^{98}$, V. Zhuravlov ${ }^{99}$, R. Zimmermann ${ }^{20}$, S. Zimmermann ${ }^{20}$, S. Zimmermann ${ }^{48}$, M. Ziolkowski ${ }^{141}$, L. Živković ${ }^{34}$, G. Zobernig ${ }^{172}$, A. Zoccoli ${ }^{19 a, 19 b}$, M. zur Nedden ${ }^{15}$, V. Zutshi ${ }^{106}$

${ }^{1}$ University at Albany, 1400 Washington Ave, Albany, NY 12222, United States of America

${ }^{2}$ University of Alberta, Department of Physics, Centre for Particle Physics, Edmonton, AB T6G 2G7, Canada

${ }^{3}$ Ankara University ${ }^{(a)}$, Faculty of Sciences, Department of Physics, TR 061000 Tandogan, Ankara; Dumlupinar University $^{(\mathrm{b})}$, Faculty of Arts and Sciences, Department of Physics, Kutahya; Gazi University ${ }^{(\mathrm{c})}$, Faculty of Arts and Sciences, Department of Physics, 06500, Teknikokullar, Ankara; TOBB University of Economics and Technology ${ }^{(\mathrm{d})}$, Faculty of Arts and Sciences, Division of Physics, 06560, Sogutozu, Ankara; Turkish Atomic Energy Authority (e), 06530, Lodumlu, Ankara, Turkey

${ }^{4}$ LAPP, Université de Savoie, CNRS/IN2P3, Annecy-le-Vieux, France

${ }^{5}$ Argonne National Laboratory, High Energy Physics Division, 9700 S. Cass Avenue, Argonne, IL 60439, United States of America

${ }^{6}$ University of Arizona, Department of Physics, Tucson, AZ 85721, United States of America

${ }^{7}$ The University of Texas at Arlington, Department of Physics, Box 19059, Arlington, TX 76019, United States of America

${ }^{8}$ University of Athens, Nuclear \& Particle Physics, Department of Physics, Panepistimiopouli, Zografou, GR 15771 Athens, Greece

${ }^{9}$ National Technical University of Athens, Physics Department, 9-Iroon Polytechniou, GR 15780 Zografou, Greece

${ }^{10}$ Institute of Physics, Azerbaijan Academy of Sciences, H. Javid Avenue 33, AZ 143 Baku, Azerbaijan

${ }^{11}$ Institut de Física d'Altes Energies, IFAE, Edifici Cn, Universitat Autònoma de Barcelona, ES - 08193 Bellaterra (Barcelona), Spain

${ }^{12}$ University of Belgrade ${ }^{(a)}$, Institute of Physics, P.O. Box 57, 11001 Belgrade; Vinca Institute of Nuclear Sciences ${ }^{(b)}$, M. Petrovica Alasa 12-14, 11000 Belgrade, Serbia

${ }^{13}$ University of Bergen, Department for Physics and Technology, Allegaten 55, NO - 5007 Bergen, Norway

${ }^{14}$ Lawrence Berkeley National Laboratory and University of California, Physics Division, MS50B-6227, 1 Cyclotron Road, Berkeley, CA 94720, United States of America

${ }^{15}$ Humboldt University, Institute of Physics, Berlin, Newtonstr. 15, D-12489 Berlin, Germany 
${ }^{16}$ University of Bern, Albert Einstein Center for Fundamental Physics, Laboratory for High Energy Physics, Sidlerstrasse 5, CH - 3012 Bern, Switzerland

${ }^{17}$ University of Birmingham, School of Physics and Astronomy, Edgbaston, Birmingham B15 2TT, United Kingdom

${ }^{18}$ Bogazici University ${ }^{(\mathrm{a})}$, Faculty of Sciences, Department of Physics, TR - 80815 Bebek-Istanbul; Dogus University ${ }^{(\mathrm{b})}$,

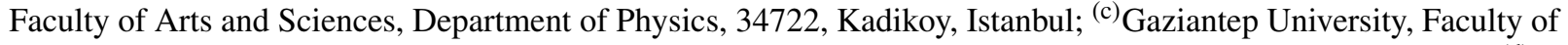
Engineering, Department of Physics Engineering, 27310, Sehitkamil, Gaziantep; Istanbul Technical University ${ }^{(d)}$, Faculty of Arts and Sciences, Department of Physics, 34469, Maslak, Istanbul, Turkey

${ }^{19}$ INFN Sezione di Bologna ${ }^{(\mathrm{a})}$; Università di Bologna, Dipartimento di Fisica ${ }^{(\mathrm{b})}$, viale C. Berti Pichat, 6/2, IT - 40127 Bologna, Italy

${ }^{20}$ University of Bonn, Physikalisches Institut, Nussallee 12, D - 53115 Bonn, Germany

${ }^{21}$ Boston University, Department of Physics, 590 Commonwealth Avenue, Boston, MA 02215, United States of America

${ }^{22}$ Brandeis University, Department of Physics, MS057, 415 South Street, Waltham, MA 02454, United States of America

${ }^{23}$ Universidade Federal do Rio De Janeiro, COPPE/EE/IF ${ }^{(a)}$, Caixa Postal 68528, Ilha do Fundao, BR - 21945-970 Rio de Janeiro; ${ }^{(b)}$ Universidade de Sao Paulo, Instituto de Fisica, R.do Matao Trav. R.187, Sao Paulo - SP, 05508 - 900, Brazil

${ }^{24}$ Brookhaven National Laboratory, Physics Department, Bldg. 510A, Upton, NY 11973, United States of America

${ }^{25}$ National Institute of Physics and Nuclear Engineering ${ }^{(a)}$, Str. Atomistilor 407, P.O. Box MG-6, R-077125, Bucharest-Magurele; University Politehnica Bucharest $^{\left({ }^{b}\right)}$, Rectorat - AN 001, 313 Splaiul Independentei, sector 6, 060042 Bucuresti; West University in Timisoara ${ }^{(\mathrm{c})}$, Bd. Vasile Parvan 4, Timisoara, Romania

${ }^{26}$ Universidad de Buenos Aires, FCEyN, Dto. Fisica, Pab I - C. Universitaria, 1428 Buenos Aires, Argentina

${ }^{27}$ University of Cambridge, Cavendish Laboratory, J J Thomson Avenue, Cambridge CB3 0HE, United Kingdom

${ }^{28}$ Carleton University, Department of Physics, 1125 Colonel By Drive, Ottawa ON K1S 5B6, Canada

${ }^{29}$ CERN, CH - 1211 Geneva 23, Switzerland

${ }^{30}$ University of Chicago, Enrico Fermi Institute, 5640 S. Ellis Avenue, Chicago, IL 60637, United States of America

${ }^{31}$ Pontificia Universidad Católica de Chile, Facultad de Fisica, Departamento de Fisica ${ }^{(a)}$, Avda. Vicuna Mackenna 4860, San Joaquin, Santiago; Universidad Técnica Federico Santa María, Departamento de Física ${ }^{(b)}$, Avda. Espãna 1680, Casilla 110-V, Valparaíso, Chile

${ }^{32}$ Institute of High Energy Physics, Chinese Academy of Sciences ${ }^{(a)}$, P.O. Box 918, 19 Yuquan Road, Shijing Shan District, CN - Beijing 100049; University of Science \& Technology of China (USTC), Department of Modern Physics ${ }^{\left({ }^{b}\right)}$, Hefei, CN - Anhui 230026; Nanjing University, Department of Physics ${ }^{(\mathrm{c})}$, Nanjing, CN - Jiangsu 210093; Shandong University, High Energy Physics Group ${ }^{(d)}$, Jinan, CN - Shandong 250100, China

${ }^{33}$ Laboratoire de Physique Corpusculaire, Clermont Université, Université Blaise Pascal, CNRS/IN2P3, FR - 63177 Aubiere Cedex, France

${ }^{34}$ Columbia University, Nevis Laboratory, 136 So. Broadway, Irvington, NY 10533, United States of America

${ }^{35}$ University of Copenhagen, Niels Bohr Institute, Blegdamsvej 17, DK - 2100 Kobenhavn 0, Denmark

${ }^{36}$ INFN Gruppo Collegato di Cosenza ${ }^{(a)}$; Università della Calabria, Dipartimento di Fisica ${ }^{(b)}$, IT-87036 Arcavacata di Rende, Italy

${ }^{37}$ Faculty of Physics and Applied Computer Science of the AGH-University of Science and Technology, (FPACS, AGH-UST), al. Mickiewicza 30, PL-30059 Cracow, Poland

${ }^{38}$ The Henryk Niewodniczanski Institute of Nuclear Physics, Polish Academy of Sciences, ul. Radzikowskiego 152, PL - 31342 Krakow, Poland

${ }^{39}$ Southern Methodist University, Physics Department, 106 Fondren Science Building, Dallas, TX 75275-0175, United States of America

${ }^{40}$ University of Texas at Dallas, 800 West Campbell Road, Richardson, TX 75080-3021, United States of America

${ }^{41}$ DESY, Notkestr. 85, D-22603 Hamburg and Platanenallee 6, D-15738 Zeuthen, Germany

${ }^{42}$ TU Dortmund, Experimentelle Physik IV, DE - 44221 Dortmund, Germany

${ }^{43}$ Technical University Dresden, Institut für Kern- und Teilchenphysik, Zellescher Weg 19, D-01069 Dresden, Germany

${ }^{44}$ Duke University, Department of Physics, Durham, NC 27708, United States of America

${ }^{45}$ University of Edinburgh, School of Physics \& Astronomy, James Clerk Maxwell Building, The Kings Buildings, Mayfield Road, Edinburgh EH9 3JZ, United Kingdom

${ }^{46}$ Fachhochschule Wiener Neustadt; Johannes Gutenbergstrasse 3, AT - 2700 Wiener Neustadt, Austria

${ }^{47}$ INFN Laboratori Nazionali di Frascati, via Enrico Fermi 40, IT-00044 Frascati, Italy

${ }^{48}$ Albert-Ludwigs-Universität, Fakultät für Mathematik und Physik, Hermann-Herder Str. 3, D - 79104 Freiburg i.Br., Germany 
${ }^{49}$ Université de Genève, Section de Physique, 24 rue Ernest Ansermet, CH - 1211 Geneve 4, Switzerland

${ }^{50}$ INFN Sezione di Genova $^{(a)}$; Università di Genova, Dipartimento di Fisica ${ }^{(b)}$, via Dodecaneso 33, IT - 16146 Genova, Italy

${ }^{51}$ Institute of Physics of the Georgian Academy of Sciences, 6 Tamarashvili St., GE - 380077 Tbilisi; Tbilisi State University, HEP Institute, University St. 9, GE - 380086 Tbilisi, Georgia

${ }^{52}$ Justus-Liebig-Universität Giessen, II Physikalisches Institut, Heinrich-Buff Ring 16, D-35392 Giessen, Germany

${ }^{53}$ University of Glasgow, Department of Physics and Astronomy, Glasgow G12 8QQ, United Kingdom

${ }^{54}$ Georg-August-Universität, II. Physikalisches Institut, Friedrich-Hund Platz 1, D-37077 Göttingen, Germany

${ }^{55}$ Laboratoire de Physique Subatomique et de Cosmologie, CNRS/IN2P3, Université Joseph Fourier, INPG, 53 avenue des Martyrs, FR - 38026 Grenoble Cedex, France

${ }^{56}$ Hampton University, Department of Physics, Hampton, VA 23668, United States of America

${ }^{57}$ Harvard University, Laboratory for Particle Physics and Cosmology, 18 Hammond Street, Cambridge, MA 02138, United States of America

${ }^{58}$ Ruprecht-Karls-Universität Heidelberg, Kirchhoff-Institut für Physik ${ }^{(a)}$, Im Neuenheimer Feld 227, D-69120 Heidelberg; Physikalisches Institut ${ }^{(\mathrm{b})}$, Philosophenweg 12, D-69120 Heidelberg; ZITI Ruprecht-Karls-University Heidelberg ${ }^{(\mathrm{c})}$, Lehrstuhl für Informatik V, B6, 23-29, DE - 68131 Mannheim, Germany

${ }^{59}$ Hiroshima University, Faculty of Science, 1-3-1 Kagamiyama, Higashihiroshima-shi, JP - 739-8526 Hiroshima, Japan

${ }^{60}$ Hiroshima Institute of Technology, Faculty of Applied Information Science, 2-1-1 Miyake Saeki-ku, Hiroshima-shi, JP - 731-5193 Hiroshima, Japan

${ }^{61}$ Indiana University, Department of Physics, Swain Hall West 117, Bloomington, IN 47405-7105, United States of America

${ }^{62}$ Institut für Astro- und Teilchenphysik, Technikerstrasse 25, A - 6020 Innsbruck, Austria

${ }^{63}$ University of Iowa, 203 Van Allen Hall, Iowa City, IA 52242-1479, United States of America

${ }^{64}$ Iowa State University, Department of Physics and Astronomy, Ames High Energy Physics Group, Ames, IA 50011-3160, United States of America

${ }^{65}$ Joint Institute for Nuclear Research, JINR, Dubna, RU - 141980 Moscow Region, Russia

${ }^{66}$ KEK, High Energy Accelerator Research Organization, 1-1 Oho, Tsukuba-shi, Ibaraki-ken 305-0801, Japan

${ }^{67}$ Kobe University, Graduate School of Science, 1-1 Rokkodai-cho, Nada-ku, JP 657-8501 Kobe, Japan

${ }^{68}$ Kyoto University, Faculty of Science, Oiwake-cho, Kitashirakawa, Sakyou-ku, Kyoto-shi, JP - 606-8502 Kyoto, Japan

${ }^{69}$ Kyoto University of Education, 1 Fukakusa, Fujimori, fushimi-ku, Kyoto-shi, JP - 612-8522 Kyoto, Japan

${ }^{70}$ Universidad Nacional de La Plata, FCE, Departamento de Física, IFLP (CONICET-UNLP), C.C. 67, 1900 La Plata, Argentina

${ }^{71}$ Lancaster University, Physics Department, Lancaster LA1 4YB, United Kingdom

${ }^{72}$ INFN Sezione di Lecce ${ }^{(a)}$; Università del Salento, Dipartimento di Fisica ${ }^{(b)}$, Via Arnesano, IT - 73100 Lecce, Italy

${ }^{73}$ University of Liverpool, Oliver Lodge Laboratory, P.O. Box 147, Oxford Street, Liverpool L69 3BX, United Kingdom

${ }^{74}$ Jožef Stefan Institute and University of Ljubljana, Department of Physics, SI-1000 Ljubljana, Slovenia

${ }^{75}$ Queen Mary University of London, Department of Physics, Mile End Road, London E1 4NS, United Kingdom

${ }^{76}$ Royal Holloway, University of London, Department of Physics, Egham Hill, Egham, Surrey TW20 0EX, United Kingdom

${ }^{77}$ University College London, Department of Physics and Astronomy, Gower Street, London WC1E 6BT, United Kingdom

${ }^{78}$ Laboratoire de Physique Nucléaire et de Hautes Energies, Université Pierre et Marie Curie (Paris 6), Université Denis Diderot (Paris-7), CNRS/IN2P3, Tour 33, 4 place Jussieu, FR - 75252 Paris Cedex 05, France

${ }^{79}$ Fysiska institutionen, Lunds universitet, Box 118, SE - 22100 Lund, Sweden

${ }^{80}$ Universidad Autonoma de Madrid, Facultad de Ciencias, Departamento de Fisica Teorica, ES - 28049 Madrid, Spain

${ }^{81}$ Universität Mainz, Institut für Physik, Staudinger Weg 7, DE - 55099 Mainz, Germany

${ }^{82}$ University of Manchester, School of Physics and Astronomy, Manchester M13 9PL, United Kingdom

${ }^{83}$ CPPM, Aix-Marseille Université, CNRS/IN2P3, Marseille, France

${ }^{84}$ University of Massachusetts, Department of Physics, 710 North Pleasant Street, Amherst, MA 01003, United States of America

${ }^{85}$ McGill University, High Energy Physics Group, 3600 University Street, Montreal, Quebec H3A 2T8, Canada

${ }^{86}$ University of Melbourne, School of Physics, AU - Parkville, Victoria 3010, Australia

${ }^{87}$ The University of Michigan, Department of Physics, 2477 Randall Laboratory, 500 East University, Ann Arbor, MI 48109-1120, United States of America 
${ }^{88}$ Michigan State University, Department of Physics and Astronomy, High Energy Physics Group, East Lansing, MI 48824-2320, United States of America

${ }^{89}$ INFN Sezione di Milano ${ }^{(a)}$; Università di Milano, Dipartimento di Fisica ${ }^{(b)}$, via Celoria 16, IT - 20133 Milano, Italy

${ }^{90}$ B.I. Stepanov Institute of Physics, National Academy of Sciences of Belarus, Independence Avenue 68, Minsk 220072, Republic of Belarus

${ }^{91}$ National Scientific \& Educational Centre for Particle \& High Energy Physics, NC PHEP BSU, M. Bogdanovich St. 153, Minsk 220040, Republic of Belarus

${ }^{92}$ Massachusetts Institute of Technology, Department of Physics, Room 24-516, Cambridge, MA 02139, United States of America

${ }^{93}$ University of Montreal, Group of Particle Physics, C.P. 6128, Succursale Centre-Ville, Montreal, Quebec, H3C 3J7, Canada

${ }^{94}$ P.N. Lebedev Institute of Physics, Academy of Sciences, Leninsky pr. 53, RU - 117924 Moscow, Russia

${ }^{95}$ Institute for Theoretical and Experimental Physics (ITEP), B. Cheremushkinskaya ul. 25, RU 117218 Moscow, Russia

${ }^{96}$ Moscow Engineering \& Physics Institute (MEPhI), Kashirskoe Shosse 31, RU - 115409 Moscow, Russia

${ }^{97}$ Lomonosov Moscow State University Skobeltsyn Institute of Nuclear Physics (MSU SINP), 1(2), Leninskie gory, GSP-1, Moscow 119991, Russian Federation, Russia

${ }^{98}$ Ludwig-Maximilians-Universität München, Fakultät für Physik, Am Coulombwall 1, DE - 85748 Garching, Germany

${ }^{99}$ Max-Planck-Institut für Physik, (Werner-Heisenberg-Institut), Föhringer Ring 6, 80805 München, Germany

${ }^{100}$ Nagasaki Institute of Applied Science, 536 Aba-machi, JP 851-0193 Nagasaki, Japan

${ }^{101}$ Nagoya University, Graduate School of Science, Furo-Cho, Chikusa-ku, Nagoya, 464-8602, Japan

${ }^{102}$ INFN Sezione di Napoli ${ }^{(a)}$; Università di Napoli, Dipartimento di Scienze Fisiche ${ }^{(b)}$, Complesso Universitario di Monte Sant'Angelo, via Cinthia, IT - 80126 Napoli, Italy

${ }^{103}$ University of New Mexico, Department of Physics and Astronomy, MSC07 4220, Albuquerque, NM 87131, United States of America

${ }^{104}$ Radboud University Nijmegen/NIKHEF, Department of Experimental High Energy Physics, Heyendaalseweg 135, NL-6525 AJ, Nijmegen, Netherlands

${ }^{105}$ Nikhef National Institute for Subatomic Physics and University of Amsterdam, Science Park 105, 1098 XG Amsterdam, Netherlands

${ }^{106}$ Department of Physics, Northern Illinois University, LaTourette Hall Normal Road, DeKalb, IL 60115, United States of America

${ }^{107}$ Budker Institute of Nuclear Physics (BINP), RU - Novosibirsk 630 090, Russia

${ }^{108}$ New York University, Department of Physics, 4 Washington Place, New York NY 10003, United States of America

${ }^{109}$ Ohio State University, 191 West Woodruff Ave, Columbus, OH 43210-1117, United States of America

${ }^{110}$ Okayama University, Faculty of Science, Tsushimanaka 3-1-1, Okayama 700-8530, Japan

${ }^{111}$ University of Oklahoma, Homer L. Dodge Department of Physics and Astronomy, 440 West Brooks, Room 100, Norman, OK 73019-0225, United States of America

${ }^{112}$ Oklahoma State University, Department of Physics, 145 Physical Sciences Building, Stillwater, OK 74078-3072, United States of America

${ }^{113}$ Palacký University, 17.listopadu 50a, 77207 Olomouc, Czech Republic

${ }^{114}$ University of Oregon, Center for High Energy Physics, Eugene, OR 97403-1274, United States of America

${ }^{115}$ LAL, Univ. Paris-Sud, IN2P3/CNRS, Orsay, France

${ }^{116}$ Osaka University, Graduate School of Science, Machikaneyama-machi 1-1, Toyonaka, Osaka 560-0043, Japan

${ }^{117}$ University of Oslo, Department of Physics, P.O. Box 1048, Blindern, NO - 0316 Oslo 3, Norway

${ }^{118}$ Oxford University, Department of Physics, Denys Wilkinson Building, Keble Road, Oxford OX1 3RH, United Kingdom

${ }^{119}$ INFN Sezione di Pavia $^{(a)}$; Università di Pavia, Dipartimento di Fisica Nucleare e Teorica ${ }^{(b)}$, Via Bassi 6, IT-27100 Pavia, Italy

${ }^{120}$ University of Pennsylvania, Department of Physics, High Energy Physics Group, 209 S. 33rd Street, Philadelphia, PA 19104, United States of America

${ }^{121}$ Petersburg Nuclear Physics Institute, RU - 188300 Gatchina, Russia

${ }^{122}$ INFN Sezione di Pisa ${ }^{(a)}$; Università di Pisa, Dipartimento di Fisica E. Fermi ${ }^{(b)}$, Largo B. Pontecorvo 3, IT - 56127 Pisa, Italy

${ }^{123}$ University of Pittsburgh, Department of Physics and Astronomy, 3941 O’Hara Street, Pittsburgh, PA 15260, United States of America 
${ }^{124}$ Laboratorio de Instrumentacao e Fisica Experimental de Particulas - LIP ${ }^{(a)}$, Avenida Elias Garcia 14-1, PT - 1000-149 Lisboa, Portugal; Universidad de Granada, Departamento de Fisica Teorica y del Cosmos and CAFPE ${ }^{(\mathrm{b})}$, E-18071 Granada, Spain

${ }^{125}$ Institute of Physics, Academy of Sciences of the Czech Republic, Na Slovance 2, CZ - 18221 Praha 8, Czech Republic

${ }^{126}$ Charles University in Prague, Faculty of Mathematics and Physics, Institute of Particle and Nuclear Physics,

V Holesovickach 2, CZ - 18000 Praha 8, Czech Republic

${ }^{127}$ Czech Technical University in Prague, Zikova 4, CZ - 16635 Praha 6, Czech Republic

${ }^{128}$ State Research Center Institute for High Energy Physics, Moscow Region, 142281, Protvino, Pobeda street, 1, Russia

${ }^{129}$ Rutherford Appleton Laboratory, Science and Technology Facilities Council, Harwell Science and Innovation Campus, Didcot OX11 0QX, United Kingdom

${ }^{130}$ University of Regina, Physics Department, Regina, Canada

${ }^{131}$ Ritsumeikan University, Noji Higashi 1 chome 1-1, JP - Kusatsu, Shiga 525-8577, Japan

${ }^{132}$ INFN Sezione di Roma I ${ }^{(a)}$; Università La Sapienza, Dipartimento di Fisica ${ }^{(b)}$, Piazzale A. Moro 2, IT- 00185 Roma, Italy

${ }^{133}$ INFN Sezione di Roma Tor Vergata $^{(a)}$; Università di Roma Tor Vergata, Dipartimento di Fisica ${ }^{(b)}$, via della Ricerca Scientifica, IT-00133 Roma, Italy

${ }^{134}$ INFN Sezione di Roma Tre ${ }^{(\mathrm{a})}$; Università Roma Tre, Dipartimento di Fisica ${ }^{(\mathrm{b})}$, via della Vasca Navale 84, IT-00146 Roma, Italy

${ }^{135}$ Réseau Universitaire de Physique des Hautes Energies (RUPHE): Université Hassan II, Faculté des Sciences Ain Chock $^{(a)}$, B.P. 5366, MA - Casablanca; Centre National de l'Energie des Sciences Techniques Nucleaires ${\left({ }^{2}\right.}$ CNESTEN) ${ }^{(b)}$, B.P. 1382 R.P. 10001 Rabat 10001; Université Mohamed Premier ${ }^{(\mathrm{c})}$, LPTPM, Faculté des Sciences, B.P.717. Bd. Mohamed VI, 60000, Oujda; Université Mohammed V, Faculté des Sciences ${ }^{(\mathrm{d})}$, 4 Avenue Ibn Battouta, BP 1014 RP, 10000 Rabat, Morocco

${ }^{136}$ CEA, DSM/IRFU, Centre d'Etudes de Saclay, FR - 91191 Gif-sur-Yvette, France

${ }^{137}$ University of California Santa Cruz, Santa Cruz Institute for Particle Physics (SCIPP), Santa Cruz, CA 95064, United States of America

${ }^{138}$ University of Washington, Seattle, Department of Physics, Box 351560, Seattle, WA 98195-1560, United States of America

${ }^{139}$ University of Sheffield, Department of Physics \& Astronomy, Hounsfield Road, Sheffield S3 7RH, United Kingdom

${ }^{140}$ Shinshu University, Department of Physics, Faculty of Science, 3-1-1 Asahi, Matsumoto-shi, JP - Nagano 390-8621, Japan

${ }^{141}$ Universität Siegen, Fachbereich Physik, D 57068 Siegen, Germany

${ }^{142}$ Simon Fraser University, Department of Physics, 8888 University Drive, CA - Burnaby, BC V5A 1S6, Canada

${ }^{143}$ SLAC National Accelerator Laboratory, Stanford, California 94309, United States of America

${ }^{144}$ Comenius University, Faculty of Mathematics, Physics \& Informatics $^{(a)}$, Mlynska dolina F2, SK - 84248 Bratislava; Institute of Experimental Physics of the Slovak Academy of Sciences, Dept. of Subnuclear Physics ${ }^{(b)}$, Watsonova 47, SK - 04353 Kosice, Slovak Republic

${ }^{145(a)}$ University of Johannesburg, Department of Physics, PO Box 524, Auckland Park, Johannesburg 2006; ${ }^{(b)}$ School of Physics, University of the Witwatersrand, Private Bag 3, Wits 2050, Johannesburg, South Africa

${ }^{146}$ Stockholm University, Department of Physics ${ }^{(a)}$; The Oskar Klein Centre ${ }^{(b)}$, AlbaNova, SE - 10691 Stockholm, Sweden

${ }^{147}$ Royal Institute of Technology (KTH), Physics Department, SE - 10691 Stockholm, Sweden

${ }^{148}$ Stony Brook University, Department of Physics and Astronomy, Nicolls Road, Stony Brook, NY 11794-3800, United States of America

${ }^{149}$ University of Sussex, Department of Physics and Astronomy Pevensey, 2 Building, Falmer, Brighton BN1 9QH, United Kingdom

${ }^{150}$ University of Sydney, School of Physics, AU - 2006 Sydney NSW, Australia

${ }^{151}$ Insitute of Physics, Academia Sinica, TW - 11529 Taipei, Taiwan

${ }^{152}$ Technion, Israel Inst. of Technology, Department of Physics, Technion City, IL - Haifa 32000, Israel

${ }^{153}$ Tel Aviv University, Raymond and Beverly Sackler School of Physics and Astronomy, Ramat Aviv, IL - Tel Aviv 69978, Israel

${ }^{154}$ Aristotle University of Thessaloniki, Faculty of Science, Department of Physics, Division of Nuclear \& Particle Physics, University Campus, GR - 54124, Thessaloniki, Greece

${ }^{155}$ The University of Tokyo, International Center for Elementary Particle Physics and Department of Physics, 7-3-1 Hongo, Bunkyo-ku, JP - Tokyo 113-0033, Japan 
${ }^{156}$ Tokyo Metropolitan University, Graduate School of Science and Technology, 1-1 Minami-Osawa, Hachioji, Tokyo 192-0397, Japan

${ }^{157}$ Tokyo Institute of Technology, 2-12-1-H-34 O-Okayama, Meguro, Tokyo 152-8551, Japan

${ }^{158}$ University of Toronto, Department of Physics, 60 Saint George Street, Toronto M5S 1A7, Ontario, Canada

${ }^{159}$ TRIUMF $^{(\mathrm{a})}$, 4004 Wesbrook Mall, Vancouver, B.C. V6T 2A3; ${ }^{(b)}$ York University, Department of Physics and Astronomy, 4700 Keele St., Toronto, Ontario, M3J 1P3, Canada

${ }^{160}$ University of Tsukuba, Institute of Pure and Applied Sciences, 1-1-1 Tennoudai, Tsukuba-shi, JP - Ibaraki 305-8571, Japan

${ }^{161}$ Tufts University, Science \& Technology Center, 4 Colby Street, Medford, MA 02155, United States of America

${ }^{162}$ Universidad Antonio Narino, Centro de Investigaciones, Cra 3 Este No.47A-15, Bogota, Colombia

${ }^{163}$ University of California, Department of Physics \& Astronomy, Irvine, CA 92697-4575, United States of America

${ }^{164}$ INFN Gruppo Collegato di Udine ${ }^{(a)}$, IT-33100 Udine; ICTP( ${ }^{(b)}$, Strada Costiera 11, IT-34014, Trieste; Università di Udine, Dipartimento di Fisica ${ }^{(\mathrm{c})}$, via delle Scienze 208, IT - 33100 Udine, Italy

${ }^{165}$ University of Illinois, Department of Physics, 1110 West Green Street, Urbana, Illinois 61801, United States of America

${ }^{166}$ University of Uppsala, Department of Physics and Astronomy, P.O. Box 516, SE -751 20 Uppsala, Sweden

${ }^{167}$ Instituto de Física Corpuscular (IFIC) Centro Mixto UVEG-CSIC, Apdo. 22085 ES-46071 Valencia, Dept. Física At. Mol. y Nuclear; Dept. Ing. Electrónica; Univ. of Valencia, and Inst. de Microelectrónica de Barcelona

(IMB-CNM-CSIC), 08193 Bellaterra, Spain

${ }^{168}$ University of British Columbia, Department of Physics, 6224 Agricultural Road, CA - Vancouver, B.C. V6T 1Z1, Canada

${ }^{169}$ University of Victoria, Department of Physics and Astronomy, P.O. Box 3055, Victoria B.C., V8W 3P6, Canada

${ }^{170}$ Waseda University, WISE, 3-4-1 Okubo, Shinjuku-ku, Tokyo, 169-8555, Japan

${ }^{171}$ The Weizmann Institute of Science, Department of Particle Physics, P.O. Box 26, IL - 76100 Rehovot, Israel

${ }^{172}$ University of Wisconsin, Department of Physics, 1150 University Avenue, WI 53706 Madison, Wisconsin, United States of America

${ }^{173}$ Julius-Maximilians-University of Würzburg, Physikalisches Institute, Am Hubland, 97074 Würzburg, Germany

${ }^{174}$ Bergische Universität, Fachbereich C, Physik, Postfach 100127, Gauss-Strasse 20, D- 42097 Wuppertal, Germany

${ }^{175}$ Yale University, Department of Physics, PO Box 208121, New Haven CT, 06520-8121, United States of America

${ }^{176}$ Yerevan Physics Institute, Alikhanian Brothers Street 2, AM - 375036 Yerevan, Armenia

${ }^{177}$ ATLAS-Canada Tier-1 Data Centre, TRIUMF, 4004 Wesbrook Mall, Vancouver, BC, V6T 2A3, Canada

${ }^{178}$ GridKA Tier-1 FZK, Forschungszentrum Karlsruhe GmbH, Steinbuch Centre for Computing (SCC), Hermann-von-Helmholtz-Platz 1, 76344 Eggenstein-Leopoldshafen, Germany

${ }^{179}$ Port d'Informacio Cientifica (PIC), Universitat Autonoma de Barcelona (UAB), Edifici D, E-08193 Bellaterra, Spain

${ }^{180}$ Centre de Calcul CNRS/IN2P3, Domaine scientifique de la Doua, 27 bd du 11 Novembre 1918, 69622 Villeurbanne Cedex, France

${ }^{181}$ INFN-CNAF, Viale Berti Pichat 6/2, 40127 Bologna, Italy

${ }^{182}$ Nordic Data Grid Facility, NORDUnet A/S, Kastruplundgade 22, 1, DK-2770 Kastrup, Denmark

${ }^{183}$ SARA Reken- en Netwerkdiensten, Science Park 121, 1098 XG Amsterdam, Netherlands

${ }^{184}$ Academia Sinica Grid Computing, Institute of Physics, Academia Sinica, No.128, Sec. 2, Academia Rd., Nankang, Taipei, Taiwan 11529, Taiwan

${ }^{185}$ UK-T1-RAL Tier-1, Rutherford Appleton Laboratory, Science and Technology Facilities Council, Harwell Science and Innovation Campus, Didcot OX11 0QX, United Kingdom

${ }^{186}$ RHIC and ATLAS Computing Facility, Physics Department, Building 510, Brookhaven National Laboratory, Upton, New York 11973, United States of America

${ }^{a}$ Also at LIP, Portugal

${ }^{\mathrm{b}}$ Also at Faculdade de Ciencias, Universidade de Lisboa, Portugal

${ }^{c}$ Also at CPPM, Marseille, France

${ }^{\mathrm{d}}$ Also at Centro de Fisica Nuclear da Universidade de Lisboa, Portugal

eAlso at TRIUMF, Vancouver, Canada

${ }^{\mathrm{f}}$ Also at FPACS, AGH-UST, Cracow, Poland

${ }^{\mathrm{g}}$ Also at TRIUMF, Vancouver, Canada

${ }^{\mathrm{h}}$ Also at Department of Physics, University of Coimbra, Portugal

${ }^{\mathrm{i}}$ Now at CERN 


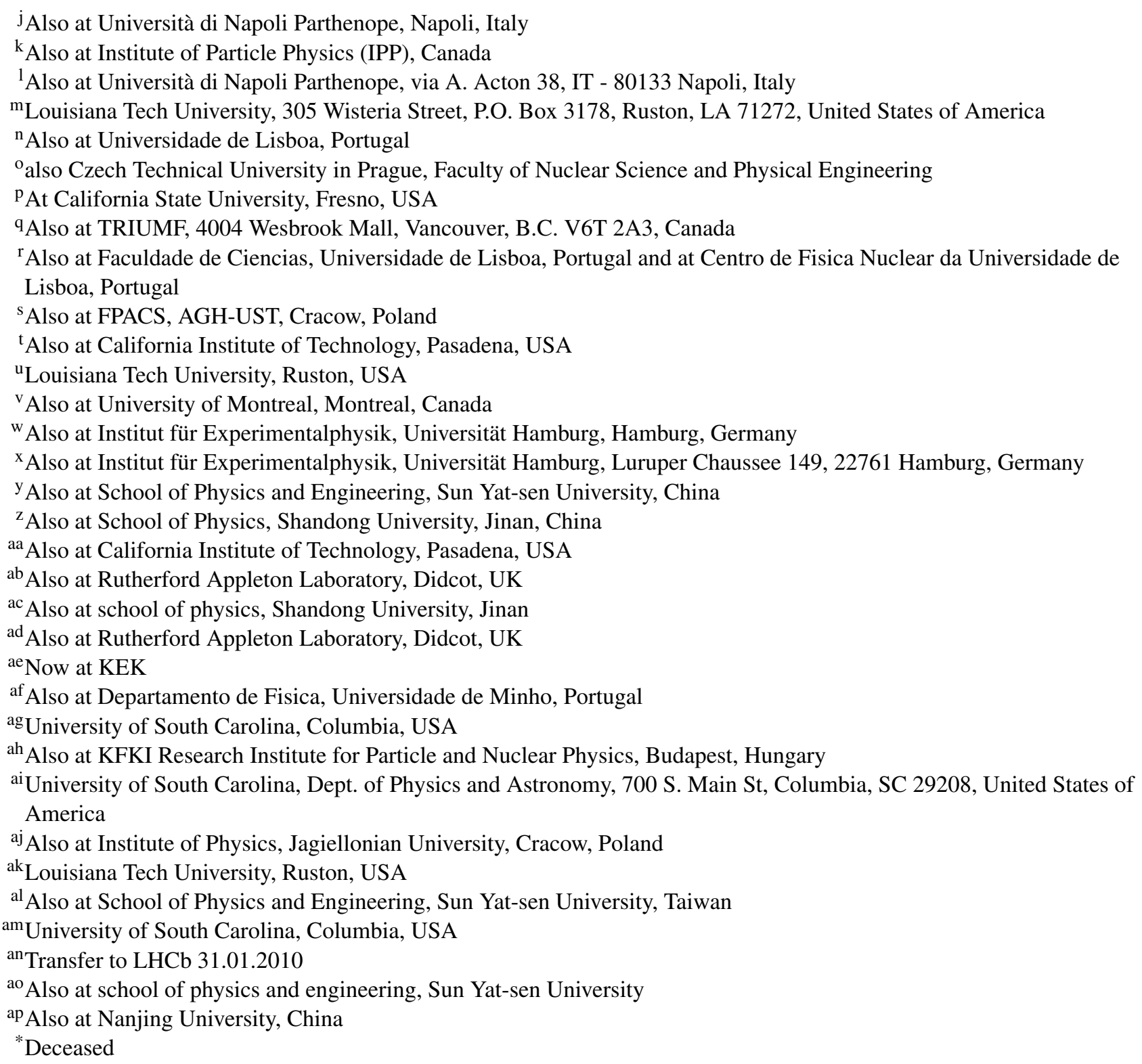

